\title{
Analytical approaches to model light transmission through turbid media
}

\author{
Leonid G. Sokoletsky ${ }^{1}$, Vladimir P. Budak ${ }^{2}$
}

1 State Key Laboratory of Satellite Ocean Environment Dynamics, Second Institute of Oceanography, State Oceanic Administration, 36 Baochu North Rd., Hangzhou 310012, Zhejiang, China; sokoletsky@sklec.ecnu.edu.cn

2 Moscow Power-Engineering Institute (Technical University), 14 Krasnokazarmennaya str., Moscow 111250, Russia

\section{ABSTRACT}

The transmission of light is one of the key optical processes in the terrestrial environment (the atmosphere and underlying surfaces). The dependence of light transmittance on the illumination/observation conditions and optical properties of the atmosphere-underlying system can be studied using the integro-differential radiative transfer equation. However, for numerous applications a set of analytical equations is needed to describe the transmitted light intensity and flux. In this paper, we describe various analytical techniques to study light transmittance through light scattering and absorbing media. A physical significance and improved mathematical accuracy of approximations are provided using the analytical models for the diffusion exponent, average cosine of the light field, spherical and plane albedos. The accuracy of various approximations is studied using exact radiative transfer calculations with various scattering phase functions, single-scattering albedos, observational conditions, and optical depths.

Keywords: spherical transmittance; plane transmittance; diffuse attenuation coefficient; spherical albedo; plane albedo; diffusion exponent; average cosine of the light field; IPOL; MDOM; SORD 


\section{Introduction}

A quantitative measurement of the light radiation propagating through a turbid medium is a key to an estimation of the turbidity level in various media such as planetary atmospheres, natural waters, biological tissues, blood, paints, dyes, paper, food, etc. For such media as the Earth's atmosphere and natural waters, level of turbidity, transmittance $(T)$ and downwelling attenuation coefficient $\left(K_{\mathrm{d}}\right)$ serve as indices of the air or water quality. Therefore, knowledge of $T$ or $K_{d}$ is extremely important for in situ and remote-sensing monitoring for the Earth's atmosphere and the world's natural waters.

However, in many cases (for example, for remote sensed observations spanning large areas of space) direct measurements of $T$ or $K_{d}$ are impossible, and may only be modeled. For this purpose, other optical properties that may be measured or more easily modeled [such as reflectance $(R)$, absorption (a), attenuation $(c)$, scattering $(b)$, backscattering $\left(b_{b}\right)$ coefficients and scattering phase function $\left.p(\theta)\right]$ are generally used. Therefore, there is a need to develop the models relating these optical properties to $T$ and $K_{d}$. Such models actually mean a solution of the radiative transfer equation (RTE) for radiation transmitted through a layer.

This problem considered for a huge variability of real situations may be complicated due to various additional factors, such as non-sphericity of atmosphere, polarized light, cloudiness, package effects, optically inhomogeneous layers, etc. Obviously, a full accounting of all these factors is an extremely difficult task, even with the use of modern computers and computer techniques. In this paper, therefore, we will limit ourselves to consideration of the general and the rather simple conditions of light propagation through a plane-parallel optically homogeneous turbid layer without edge effects, 
polarization, Raman scattering, fluorescence and any other inelastic and trans-spectral processes. However, the range of layers under investigation covers the widest possible span of optical properties and geometrical conditions for light illumination: from completely absorbing to completely scattering, from optically isotropic to almost completely forward-scattering, and from normal to near-grazing and diffuse illumination.

We consider this study as a continuation of our previous efforts of reviewing existing methods and developing new ones for evaluation of reflecting and transmitting properties of turbid layers. Table 1 provides a summary of these efforts.

Table 1. A summary of several publications devoted to modeling the reflecting and transmittance properties of turbid layers. $R, R_{\mathrm{p}}, r, T_{\mathrm{p}}, t$, and $K_{\mathrm{d}}$ are reserved here respectively for the reflection function (reflectance factor), plane albedo (directionalhemispherical reflectance), spherical albedo (bi-hemispherical reflectance), plane (directional-hemispherical) transmittance, spherical (global) transmittance, and diffuse attenuation coefficient for downwelling irradiance. The numerical methods are: IIM stands for the invariant imbedding method, (M)DOM is the (modified) discrete ordinates method, MSH is the modification of a spherical harmonics method; the analytical methods: EA (exponential approximation), GKM (Gurevich-Kubelka-Munk), GKS (Gordon-Kokhanovsky-Sokoletsky), GS (Gemert-Star), GSK (Gordon-SokoletskyKokhanovsky), HKS (Hapke-Kokhanovsky-Sokoletsky), MR (Mudgett-Richards), QSSA (quasi-single-scattering approximation), PA (polynomial approximation); "dir" and "dif" refer to completely direct and completely diffuse incident fluxes, respectively. A parameter in parentheses is the optical depth $\tau$. 


\begin{tabular}{|c|c|c|c|c|}
\hline Publication & $\begin{array}{l}\text { Benchmark } \\
\text { model }\end{array}$ & $\begin{array}{c}\text { Number of } \\
\text { approximations } \\
\text { considered }\end{array}$ & Best models & $\begin{array}{l}\text { References for the } \\
\text { best models }\end{array}$ \\
\hline $\begin{array}{l}\text { Sokoletsky } \\
\text { (2004) }\end{array}$ & Hulst & 19 for $r(\infty)$ & $\begin{array}{c}\text { Extended } \\
\text { Pierce- } \\
\text { Marcus, } \\
\text { Hirata- } \\
\text { Walker, } \\
\text { Mudgett- } \\
\text { Richards }\end{array}$ & $\begin{array}{c}\text { Mudgett and } \\
\text { Richards (1971), } \\
\text { Walker (1994), Pierce } \\
\text { and Marcus (1997), } \\
\text { Hirata (2004) }\end{array}$ \\
\hline $\begin{array}{c}\text { Sokoletsky } \\
\text { and } \\
\text { Kokhanovsky } \\
(2005)\end{array}$ & IIM & $\begin{array}{c}1 \text { for } R(\infty), 2 \text { for } \\
R_{\mathrm{p}}(\infty), 3 \text { for } \\
r(\infty)\end{array}$ & $\begin{array}{c}\text { Hulst, } \\
\text { Mudgett- } \\
\text { Richards, PA, } \\
\text { QSSA }\end{array}$ & $\begin{array}{c}\text { Gordon (1973), } \\
\text { Mudgett and } \\
\text { Richards (1971), } \\
\text { Hulst (1974), } \\
\text { Sokoletsky and } \\
\text { Kokhanovsky (2005) }\end{array}$ \\
\hline $\begin{array}{l}\text { Kokhanovsky } \\
\text { and } \\
\text { Sokoletsky } \\
\text { (2006a). }\end{array}$ & IIM & 3 for $r(\infty)$ & $\begin{array}{l}\text { Hulst, } \\
\text { Mudgett- } \\
\text { Richards }\end{array}$ & $\begin{array}{c}\text { Mudgett and } \\
\text { Richards (1971), } \\
\text { Hulst (1974) }\end{array}$ \\
\hline $\begin{array}{l}\text { Kokhanovsky } \\
\text { and } \\
\text { Sokoletsky } \\
\text { (2006b). }\end{array}$ & IIM & $\begin{array}{c}2 \text { for } R(\infty), 4 \text { for } \\
R_{\mathrm{p}}(\infty), 2 \text { for } \\
r(\infty)\end{array}$ & $\begin{array}{l}\text { EA, extended } \\
\text { Hapke, QSSA }\end{array}$ & $\begin{array}{l}\text { Gordon (1973), } \\
\text { Hapke (2012), } \\
\text { Kokhanovsky and } \\
\text { Sokoletsky (2006b) }\end{array}$ \\
\hline $\begin{array}{l}\text { Sokoletsky et } \\
\text { al. (2009) }\end{array}$ & $\begin{array}{c}\text { IIM; DOM } \\
\text { ("RADUGA"), } \\
\text { DOM+MSH }\end{array}$ & 4 for $R_{\mathrm{p}}(\infty)$ & $\begin{array}{l}\text { Extended } \\
\text { Hapke, } \\
\text { extended } \\
\text { QSSA }\end{array}$ & $\begin{array}{c}\text { Hapke (2012), } \\
\text { Sokoletsky et al. } \\
(2009)\end{array}$ \\
\hline $\begin{array}{l}\text { Sokoletsky et } \\
\text { al. (2013) }\end{array}$ & IIM & $\begin{array}{l}10 \text { for } R_{\mathrm{p}}(\infty), 28 \\
\quad \text { for } r(\infty)\end{array}$ & $\begin{array}{c}\text { Flock, } \\
\text { GKM+MR, } \\
\text { GKM-new, } \\
\text { GKS, Gordon, } \\
\text { GS, GSK } \\
\text { (QSSA), } \\
\text { Haltrin, HKS } \\
\text { 1, HKS 2 } \\
\text { (extended } \\
\text { Hapke), Hulst }\end{array}$ & $\begin{array}{c}\text { Kubelka (1948), } \\
\text { Mudgett and } \\
\text { Richards (1971), } \\
\text { Gordon (1973), } \\
\text { Golubitsky et al. } \\
\text { (1974), Hulst (1974, } \\
\text { 1980), Gordon et al. } \\
\text { (1975), Gemert and } \\
\text { Star (1987), Flock et } \\
\text { al. (1989), Gordon } \\
\text { (1989); Hapke } \\
\text { (2012), Haltrin } \\
\text { (1998); Sokoletsky } \\
\text { and Kokhanovsky } \\
\text { (2005), Kokhanovsky } \\
\text { and Sokoletsky } \\
\text { (2006b), Sokoletsky } \\
\text { et al. (2013) }\end{array}$ \\
\hline
\end{tabular}




\begin{tabular}{|c|c|c|c|c|}
\hline $\begin{array}{l}\text { Sokoletsky et } \\
\text { al. }(2014 a)\end{array}$ & MDOM & $\begin{array}{c}8 \text { for } T_{p}(\tau) \text { and } \\
K_{\mathrm{d}, \mathrm{dir}}(\tau)\end{array}$ & $\begin{array}{l}\text { Ben-David, } \\
\text { Gordon, } \\
\text { QSSA }\end{array}$ & $\begin{array}{c}\text { Gordon and Brown } \\
\text { (1974), Gordon et al. } \\
\text { (1975), Gordon } \\
\text { (1989); Ben-David } \\
(1995,1997)\end{array}$ \\
\hline $\begin{array}{c}\text { Sokoletsky } \\
\text { and Budak } \\
(2016 a)\end{array}$ & MDOM & $\begin{array}{c}15 \text { for } T_{\mathrm{p}}(\tau), t(\tau), \\
K_{\mathrm{d}, \mathrm{dir}}(\tau), \text { and } \\
K_{\mathrm{d}, \mathrm{dif}}(\tau)\end{array}$ & $\begin{array}{l}\text { Ben-David, } \\
\text { Cornet, } \\
\text { Haltrin, QSSA }\end{array}$ & $\begin{array}{c}\text { Gordon et al. (1975), } \\
\text { Cornet et al. (1992), } \\
\text { Ben-David (1995, } \\
\text { 1997), Haltrin (1998) }\end{array}$ \\
\hline $\begin{array}{l}\text { Sokoletsky } \\
\text { and Budak } \\
(2016 \mathrm{~b})\end{array}$ & MDOM & $\begin{array}{c}21 \text { for } T_{\mathrm{p}}(\tau), t(\tau), \\
K_{\mathrm{d}, \mathrm{dir}}(\tau), \text { and } \\
K_{\mathrm{d}, \text { dif }}(\tau)\end{array}$ & $\begin{array}{l}\text { Gershun, } \\
\text { HBD, QSSA, } \\
\text { Rogatkin, } \\
\text { Sobolev }\end{array}$ & $\begin{array}{c}\text { Gershun (1936), } \\
\text { Sobolev (1956), Hulst } \\
\text { (1974, 1980), Gordon } \\
\text { et al. (1975), Ben- } \\
\text { David (1995, 1997), } \\
\text { Rogatkin (2007), } \\
\text { Barun and Ivanov } \\
\text { (2011) }\end{array}$ \\
\hline $\begin{array}{l}\text { Sokoletsky } \\
\text { and Budak } \\
(2017)\end{array}$ & MDOM & $\begin{array}{c}21 \text { for } T_{p}(\tau), t(\tau), \\
K_{\mathrm{d}, \mathrm{dir}}(\tau), \text { and } \\
K_{\mathrm{d}, \text { dif }}(\tau)\end{array}$ & $\begin{array}{l}\text { Gershun, } \\
\text { HBD, QSSA, } \\
\text { Rogatkin, } \\
\text { Sobolev }\end{array}$ & $\begin{array}{c}\text { Gershun (1936), } \\
\text { Sobolev (1956), Hulst } \\
\text { (1974, 1980), Gordon } \\
\text { et al. (1975), Ben- } \\
\text { David (1995, 1997), } \\
\text { Rogatkin (2007), } \\
\text { Barun and Ivanov } \\
\text { (2011) }\end{array}$ \\
\hline
\end{tabular}

Thus, this table shows that these publications have mainly focused on reflectance of the infinite or semi-infinite layers. Far less attention has been devoted to analysis of transmitted-based analytical approximations for the given conditions of illumination/observation and a given optical depth $\tau=c z$, where $z$ is the geometric depth within the layer. The present study is intended to fill this gap.

This paper consists of five main sections. Section 2 provides a brief statement of the problem. The following section describes different solutions for the problem, both existing and new ones. Section 4 discusses the results of model calculations for both direct and diffuse illumination and considers modeling of the combined illumination. In Section 5 we summarize and discuss our results. 


\section{Statement of the Problem}

Figure 1 is a schematic diagram of light distribution in the plane-parallel layer. We assume a semi-infinite or infinite layer with a non-reflecting bottom surface. Light enters from the outside medium (for example, air) into the turbid layer, changing incident angle (relative to the normal to surface) from $\theta_{\circ}$ ("outside angle") to $\theta_{i}$ ("inside angle") after refraction on the boundary between the two media. Then photons begin to travel within the turbid layer, striking molecules, cells and particles, being partially absorbed or changing their direction of movement, i.e., being scattered. The last process, scattering, significantly changes the initial direction $\left(\mu_{i}=\cos \theta_{i}\right)$ of photons (Fig. 1), leading to an increase path length of photons.

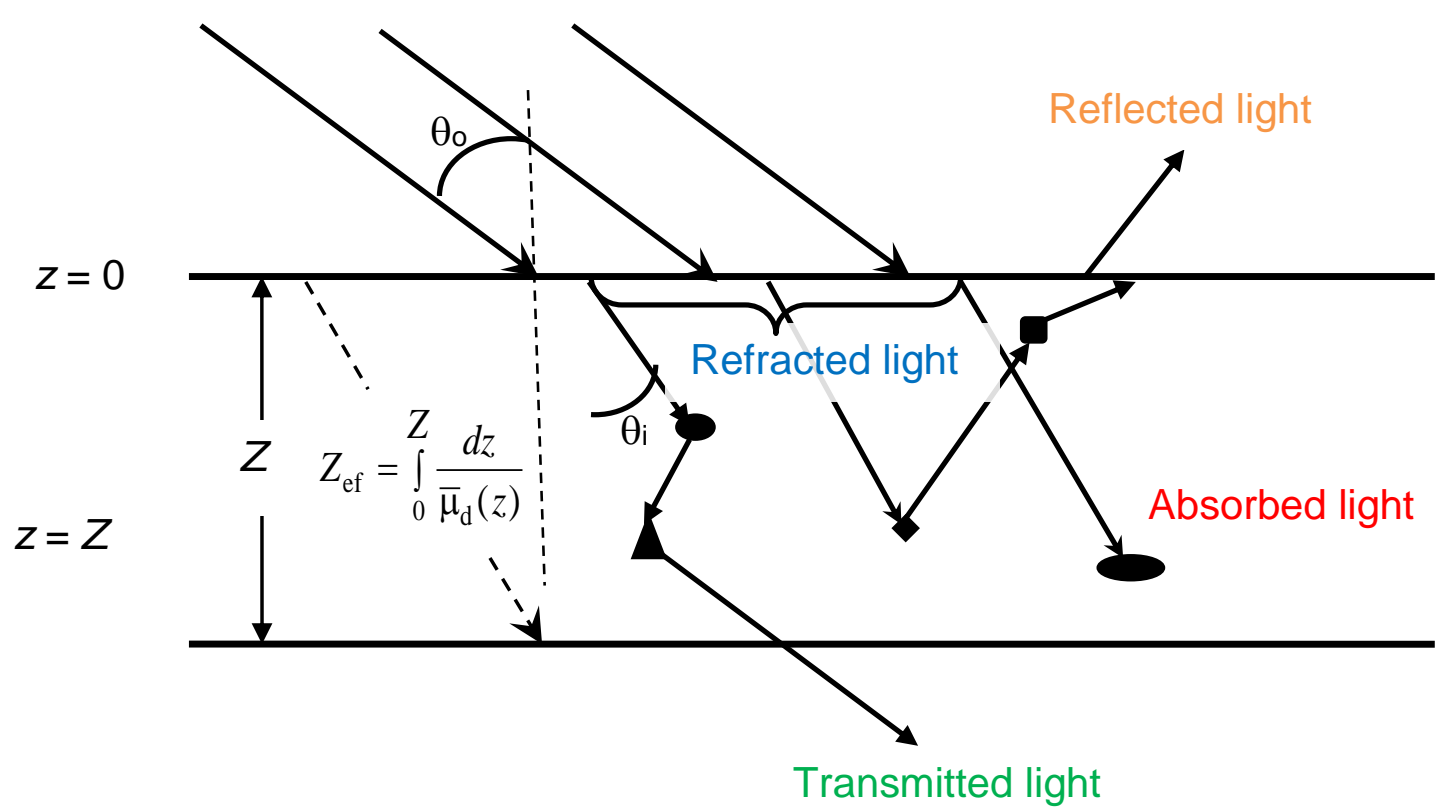

Fig. 1. A schematic geometry of movement of IIght photons in a plane-parallel turbid layer illuminated from above. The $\theta_{0}$ is the outside angle before the light's refraction on the layer's boundary; $\theta_{\mathrm{i}}$ is the angle at which light enters the layer; $Z_{\mathrm{ef}}$ is the total (or 
optically effective) path length; and $\bar{\mu}_{\mathrm{d}}(\mathrm{z})$ is the average cosine of the layer's light field for the downwelling irradiance at the geometrical depth $z$.

Following Fermat's principle, the total path length of photons may be presented as an integral $\int_{0}^{Z} \frac{d z}{\bar{\mu}_{d}(z)}$, where $z$ is the partial thickness traversed by light photons from one medium's object to another; $Z$ is the total geometrical thickness of the layer, and $\bar{\mu}_{\mathrm{d}}(\mathrm{z})$ is the average cosine of the layer's light field for the downwelling irradiance $\left(E_{d}\right)$ at depth z, defined as follows (Gordon, 1989; Kirk, 1991; Sathyendranath and Platt, 1997):

$$
\bar{\mu}_{\mathrm{d}}(\mathrm{z})=\frac{E_{\mathrm{d}}(z)}{E_{0 \mathrm{~d}}(z)}=\frac{\int_{\theta=0}^{\pi / 2} \int_{\phi=0}^{2 \pi} L(z, \theta, \phi) \sin \theta \cos \theta \mathrm{d} \phi d \theta}{\int_{\theta=0}^{\pi / 2} \int_{\phi=0}^{2 \pi} L(z, \theta, \phi) \sin \theta \mathrm{d} \theta},
$$

where $E_{0 \mathrm{~d}}(z)$ is the downwelling scalar irradiance at depth $z ; L(z, \theta, \phi)$ is the radiance at the depth $z$ traveling in direction $(\theta, \phi)$, where $\theta$ is the zenith angle and $\phi$ is the azimuth angle.

Alternatively, $\bar{\mu}_{\mathrm{d}}(\mathrm{z})$ can be expressed through the incident light angle $\mu_{\mathrm{i}}$ and such inherent optical properties (IOPs) as the beam scattering coefficient $b$, single-scattering albedo $\omega_{0}=\mathrm{b} /(a+b)=b / c$ ( $a$ and $c$ are the beam absorption and attenuation coefficients, respectively), and the average cosine of the scattering angle $\theta$ ("asymmetry parameter") $g=<\cos \theta>$ (King and Harshvardhan, 1986; Kirk, 1999; Sokoletsky et al., 2003). 
A problem of parameterization of $\bar{\mu}_{\mathrm{d}}(\mathrm{z})$ through $\mu_{\mathrm{i}}$ and IOPs is closely related to the problem of estimation $E_{\mathrm{d}}$ at given depth $z$. Let's the latter optical property is represented in the form (Siegel and Dickey, 1987; Sathyendranath and Platt, 1997):

$$
E_{\mathrm{d}}(z)=E_{\mathrm{d}}(0-) \exp \left[-\bar{K}_{\mathrm{d}}(0-\rightarrow z) z\right]
$$

where $\bar{K}_{\mathrm{d}}(0-\rightarrow z)$ is the average attenuation coefficient for downwelling irradiance $E_{\mathrm{d}}(z)$ within the depth interval from 0- ("0-" means a level just below the interface between outside and inside mediums $)$ to $z$. Then $\bar{K}_{\mathrm{d}}(0-\rightarrow z)$ may be expressed as a function of the instantaneous downwelling attenuation coefficient at depth $z$, $K_{\mathrm{d}}(z)=-\frac{d \ln E_{\mathrm{d}}(z)}{d z}:$

$$
\bar{K}_{\mathrm{d}}(0-\rightarrow z)=\frac{1}{z} \int_{0-}^{z} K_{\mathrm{d}}\left(z^{\prime}\right) \mathrm{d} z^{\prime}
$$

From Eq. (1) there follows a direct relationship between the transmittance $T(z)=$ $E_{\mathrm{d}}(\mathrm{z}) / E_{\mathrm{d}}(0-)$ and $\bar{K}_{\mathrm{d}}(0-\rightarrow z)$ :

$$
T(z)=\exp \left[-\bar{K}_{\mathrm{d}}(0-\rightarrow z) z\right]
$$

or, vice versa,

$$
\bar{K}_{\mathrm{d}}(0-\rightarrow z)=-\frac{\ln T(z)}{z}
$$

Rather than a geometric depth $z$, an optical geometrical property, namely, an optical depth $\tau=\frac{1}{z} \int_{0}^{z} c\left(z^{\prime}\right) \mathrm{d} z^{\prime}$, will be used in this study. For an optically homogeneous medium, i.e., a medium in which $a(z)=$ const, $b(z)=$ const, and, hence $c(z)=a(z)+b(z)=$ const, $\tau$ becomes simply a product of $c z$. Then Eqs. (4) and (5) may be rewritten as 


$$
T(\tau)=\exp \left[-\frac{\bar{K}_{\mathrm{d}}(0-\rightarrow \tau)}{c} \tau\right]
$$

and

$$
\frac{\bar{K}_{\mathrm{d}}(0-\rightarrow \tau)}{c}=-\frac{\ln T(\tau)}{\tau}
$$

We will subsequently use these optical quantities, comparing analytical approximations with the numerical solutions of the RTE. Two different conditions of illumination/observation will be considered: 1) Direct illumination/diffuse observation and 2) diffuse illumination/diffuse observation.

In the first case, the transmittance is considered as a plane transmittance (other names accepted in the literature are: directional-hemispherical, hemispherical or diffuse transmittance) $T_{\mathrm{p}}$, defined as follows (King and Harshvardhan, 1986; Kokhanovsky, 2007)

$$
T_{\mathrm{p}}\left(\mu_{\mathrm{i}}, \tau\right)=\frac{1}{\pi} \int_{0}^{2 \pi} \int_{0}^{1} T\left(\mu_{\mathrm{i}}, \mu_{\mathrm{v}}, \varphi, \tau\right) \mu_{\mathrm{v}} \mathrm{d} \mu_{\mathrm{v}} \mathrm{d} \varphi+\exp \left(-\tau / \mu_{\mathrm{i}}\right)
$$

where $T\left(\mu_{\mathrm{i}}, \mu_{\mathrm{v}}, \varphi, \tau\right)$ is the transmission function, defined as the ratio of direct radiation transmitted to the layer's depth $z$ relative to the incoming direct radiation; $\mu_{\mathrm{i}}=\cos \theta_{\mathrm{i}}, \mu_{\mathrm{v}}$ is the cosine of the viewing angle $\theta_{v}$ in the medium, and $\varphi$ is the azimuthal angle between the incident and scattered beam directions.

In the second case, transmittance is called spherical transmittance (other names are hemispherical-hemispherical or global transmittance) $t$, defined as (King and Harshvardhan, 1986; Kokhanovsky, 2007):

$$
t(\tau)=2 \int_{0}^{1} T_{\mathrm{p}}\left(\mu_{\mathrm{i}}, \tau\right) \mu_{\mathrm{i}} \mathrm{d} \mu_{\mathrm{i}}
$$


Therefore, four optical properties, namely, $T_{\mathrm{p}}(\mu \mathrm{i}, \quad \tau), \quad t(\tau)$, $\bar{K}_{\mathrm{d} \text {, dir }}\left(\mu_{\mathrm{i}}, 0-\rightarrow \tau\right) / c=-\ln T_{\mathrm{p}}\left(\mu_{\mathrm{i}}, \tau\right) / \tau$, and $\bar{K}_{\mathrm{d} \text {, dif }}(0-\rightarrow \tau) / c=-\ln t(\tau) / \tau$ will be the focus of our study. All of these may be calculated numerically through the scattering phase function $p(\theta)$ and the single-scattering albedo $\omega_{0}$. However, it is well known that transmittance (and also reflectance) characteristics may be calculated approximately without knowledge of full $p(\theta)$, but with a knowledge of its asymmetry parameter $g$ or the backscattering probability $B=b_{b} / b$. In turn, $p(\theta), g$, and $B$ may be expressed via refractive indices, sizes and forms of objects (molecules, cells, and particles) of the medium and optical properties of the medium itself. Assuming, for instance, a spherical shape of the layer's objects, $p(\theta), g$, and $B$ may be retrieved by applying the Mie theory (Mie, 1908) based on knowledge of refractive indices and size distributions of particles (cells, molecules). The input parameters used in this study for calculation of $p(\theta)$ and the other optical properties are presented in Table 2.

Table 2. Four datasets used for calculation of scattering phase functions $p(\theta)$ and the other optical properties. The $r_{\mathrm{ef}}$ is the effective radius of the modeled gamma particle size distribution $f(r)=\left(9 / r_{\mathrm{ef}}\right)^{7} r^{6} \exp \left(-9 r / r_{\mathrm{ef}}\right) / \Gamma(7)$ ( $\Gamma$ is the symbol for the gamma function), defined as the ratio of the third to the second moment of $f(r) ; n$ and $k$ are the real and imaginary parts of the refractive index (at wavelength of $550 \mathrm{~nm}$ ), respectively; $g=0.5 \int_{0}^{\pi} p(\theta) \sin \theta \cos \theta d \theta$ is the asymmetry parameter; $B=b_{b} / b=0.5 \int_{\pi / 2}^{\pi} p(\theta) \sin \theta d \theta$ and $F=1-B$ are the backscattering and forward scattering probabilities, respectively. 


\begin{tabular}{ccccccc}
\hline $\boldsymbol{p}(\boldsymbol{\theta})$ & $\begin{array}{c}\boldsymbol{r e f}^{\mathrm{n}} \\
(\boldsymbol{\mu \mathrm { m }})\end{array}$ & $\boldsymbol{n}$ & $\boldsymbol{k}$ & $\boldsymbol{g}$ & $\boldsymbol{B}$ & $\boldsymbol{F}$ \\
\hline $\mathbf{1}$ & 0.006 & 1.2 & 0 & 0.001933 & 0.4986 & 0.5014 \\
$\mathbf{2}$ & 0.116 & 1.25 & 0.001 & 0.5033 & 0.1559 & 0.8441 \\
$\mathbf{3}$ & 5 & 1.2 & 0.01 & 0.9583 & 0.0087 & 0.9913 \\
$\mathbf{4}$ & 6 & 1.2 & 0 & 0.8577 & 0.0398 & 0.9602 \\
\hline
\end{tabular}

For numerical computations, $p(\theta)$ is represented by the finite Fourier-Legendre series (Hulst, 1980, Ch. 6; Sokoletsky et al., 2009; O'Neil, 2017, Ch. 8), namely:

$$
p(\theta)=\sum_{\mathrm{i}=0}^{N} x_{\mathrm{i}} P_{\mathrm{i}}(\cos \theta),
$$

where $x_{\mathrm{i}}$ and $P_{\mathrm{i}}(\cos \theta)$ are Fourier-Legendre coefficients ("expansion coefficients") and Legendre polynomials, respectively, derived to obey the normalization condition:

$$
\int_{0}^{\pi} p(\theta) \sin \theta \mathrm{d} \theta=2 .
$$

The orders $N$ were found to be $5,13,469$, and 555 respectively for $p(\theta)$ numbered 1 to 4 (Table 2). Plots of $f(r)$ and $p(\theta)$ for selected scattering phase functions are shown in Figs. 2 and 3 . Note that the modal radii $\left(r_{\mathrm{m}}\right)$ of $f(r)$ are less than the effective radii $\left(r_{\mathrm{ef}}\right)$; more precisely, $r_{m}=(2 / 3) r_{\text {ef }}$ for selected gamma particle size distribution $f(r)$. 


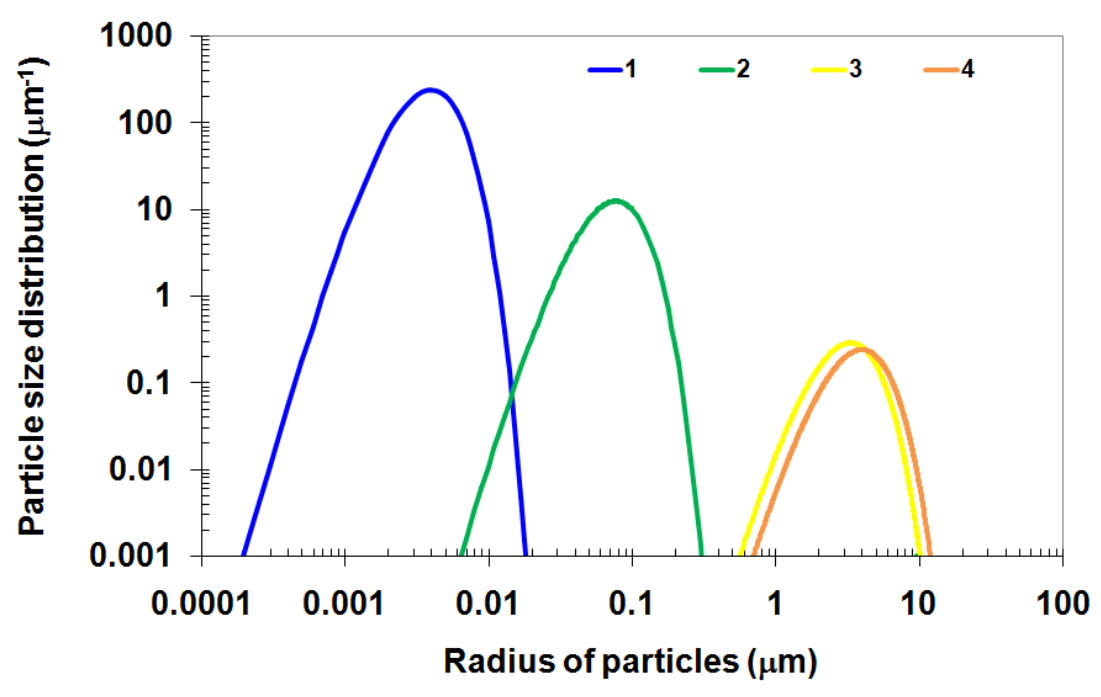

Fig. 2. Four particulate size density distributions used for the modeling. Numbers in the legend correspond to the numbers in the leftmost column of Table 2.

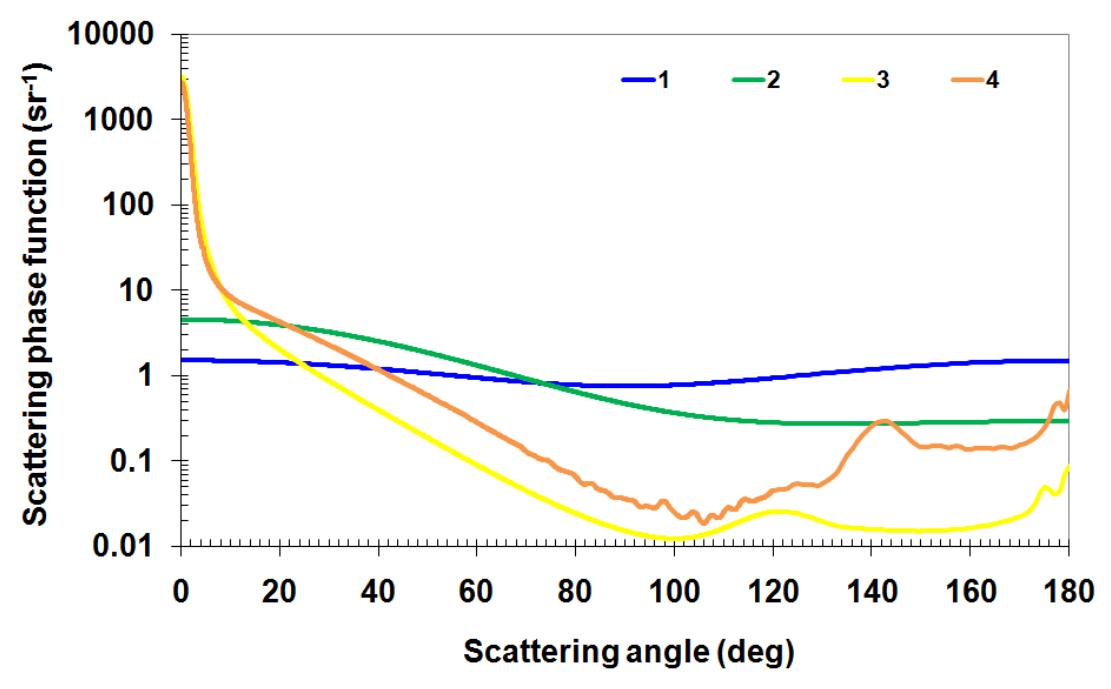

Fig. 3. Four scattering phase functions $p(\theta)$ used for modeling. Numbers in the legend corresponded to the numbers in Table 2.

The first three $p(\theta)$ were selected for the model development, while the last (\#4) was used for the validation of the model. The first $p(\theta)$ matches the molecular (Rayleigh) 
scattering, while the phase functions \#2-4 are more strongly elongated in the forward direction. $p(\theta) \# 2$ and 3 may be represented by small and large oceanic particles, respectively (Twardowski et al., 2001, 2007; Sokoletsky and Fang, 2014). Additionally, $p(\theta) \# 2$ may be applied for biomass burning aerosols (Dubovik et al., 2002), while $p(\theta)$ \#4 refers to Deirmendjian's water cloud C1 model (Deirmendjian, 1969; Kokhanovsky, 2006; Sun et al., 2006). Finally, $p(\theta) \# 3$ and 4 are suitable for strongly forward-scattering biological tissues (Prahl, 1995).

The other input parameters were selected as follows:

- illumination was assumed to be diffuse or direct; for the latter, the incidence angle of radiation $\theta_{\mathrm{i}}$ is $0^{\circ}, 5^{\circ}, 10^{\circ}, \ldots, 85^{\circ}, 89^{\circ}, 89.9^{\circ}, 89.99^{\circ}(21$ values $)$;

- optical depth $\tau$ of $0.001,0.002, \ldots, 0.01,0.02, \ldots, 0.1,0.2, \ldots, 1,2, \ldots, 10,20, \ldots, 100$, $200, \ldots, 1000,2000, \ldots, 10000$ (64 values);

- single-scattering albedo $\omega_{0}$ of $0.0001,0.1,0.2, \ldots, 0.9,0.95,0.99,0.999,0.9999$ (14 values).

Therefore, the selected parameters span an extremely wide range of angular and optical parameters, as is necessary for a deep investigation of both well-known and newly developed transmittance approximations.

\section{Solutions of the Problem}

\subsection{Numerical Methods}

Three numerical methods for calculation of $T_{\mathrm{p}}(\mu \mathrm{i}, \tau)$ and $t(\tau)$ were exploited for this study: 
- Modified discrete ordinates method (MDOM) (Budak and Korkin, 2008; Sokoletsky et al., 2009; Kokhanovsky et al., 2010);

- Intensity and POLarization (IPOL), based on the standard discrete ordinates method with single scattering correction (Emde et al., 2015);

- Successive ORDers of Scattering (SORD) (Korkin et al., 2017).

As a focus of our study is concentrated on the analytical approximations, we will not describe these methods in detail; instead, we refer readers to the corresponding references above. Note that the IPOL is equally efficient for any $\tau$ and $\omega_{0}$. In contrast, the efficiency of the MDOM deteriorates at $\omega_{0}$ close to 1 , while the SORD method often fails at too small (appr. < 0.01) or too large (appr. $>10$ ) values of $\tau$. Fig. 4 provides examples of comparison between these three methods applied for $\bar{K}_{\text {d, dif }}(0-\rightarrow \tau) / c$ computed by Eq. (7). It is seems that the closeness between the IPOL and SORD algorithms is generally better than that between the IPOL and MDOM. In actual fact, at values of $0.01 \leq \tau \leq 10$, proximity between the IPOL and SORD is almost ideal at any $p(\theta)$ and any value of $\omega 0$. However, results derived from the IPOL and MDOM are in good correspondence with one another only for some sets of parameters $\left\{g, \omega_{0}, \tau\right\}$, for instance, $\left\{g=0.503, \omega_{0}=0.1\right.$, any $\left.\tau\right\},\left\{g=0.958, \omega_{0} \leq 0.5\right.$, any $\left.\tau\right\}$, and $\left\{g=0.958\right.$, $\omega_{0} \leq$ 0.95, $\tau \geq 10\}$ while for the other parameters differences may be relatively large. Moreover, at $\omega_{0}=0.9999$ (Fig. 4d) the MDOM yields obviously physically incorrect values at any $p(\theta)$. 

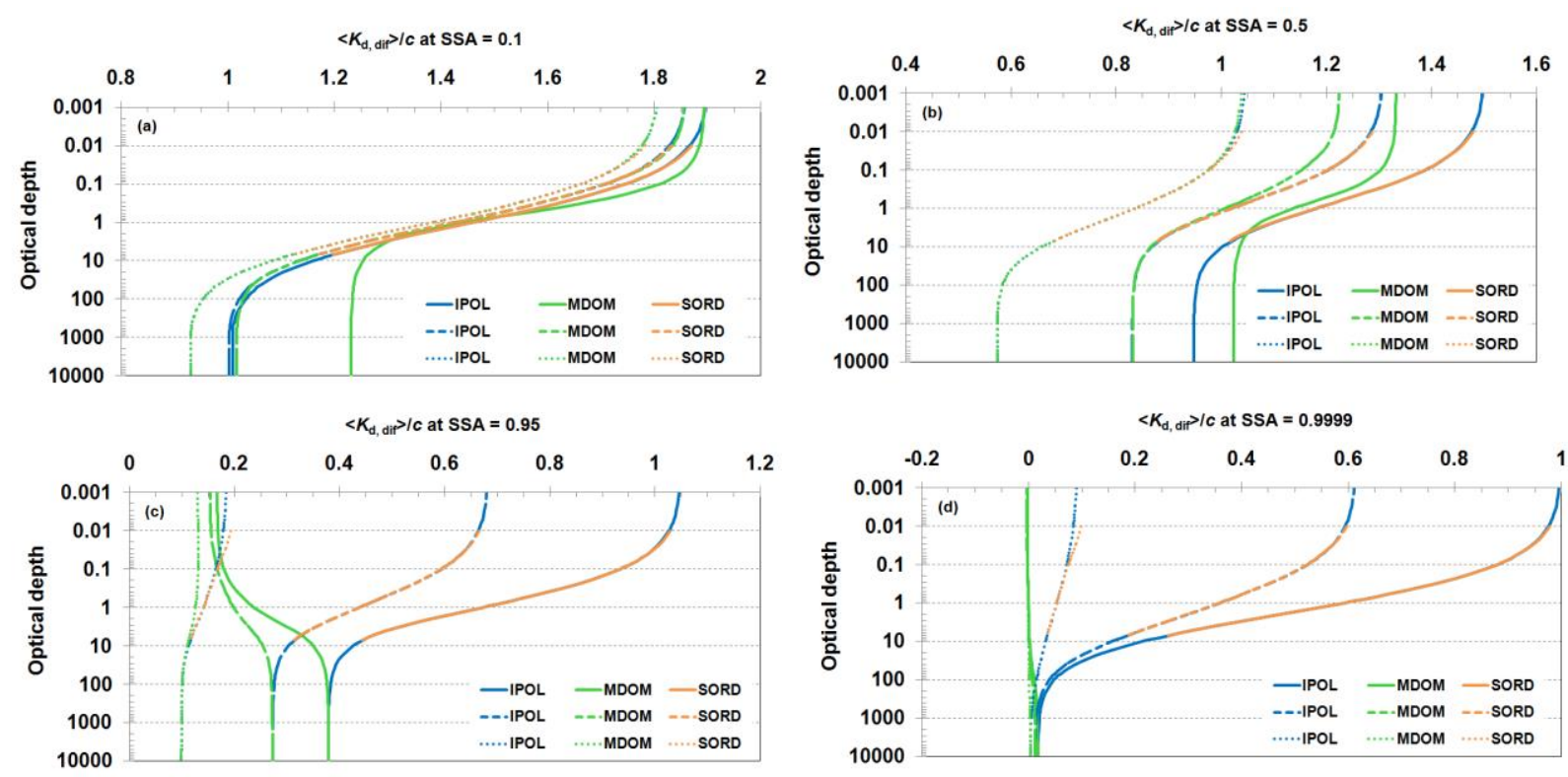

Fig. 4. A comparison of the IPOL, MDOM, and SORD (blue, green, and orange curves, respectively) numerical computations for $\bar{K}_{\mathrm{d} \text {, dif }}(0-\rightarrow \tau) / c$. Computations were performed for three $p(\theta)$ : with $g=0.002,0.503$, and 0.958 (solid, dash, and dot curves, respectively) and for four values of $\omega_{0}:$ (a) 0.1 , (b) 0.5 , (c) 0.95 , and (d) 0.9999 .

Nevertheless, a comparison between the IPOL and MDOM for $\bar{K}_{\mathrm{d}, \text { dir }}(0-\rightarrow \tau) / c$ (Fig. 5) shows much better correspondence between these two methods in the entire range of $0 \leq \tau \leq 100$. Fig. 5 demonstrates this for the one value of $\theta_{i}=30^{\circ}$. However, discrepancies sharply increased at some sets of optical parameters starting from $\tau \approx$ 100 , what can be explained by the lost of accuracy in the MDOM computer computations at large values of $\tau$. 

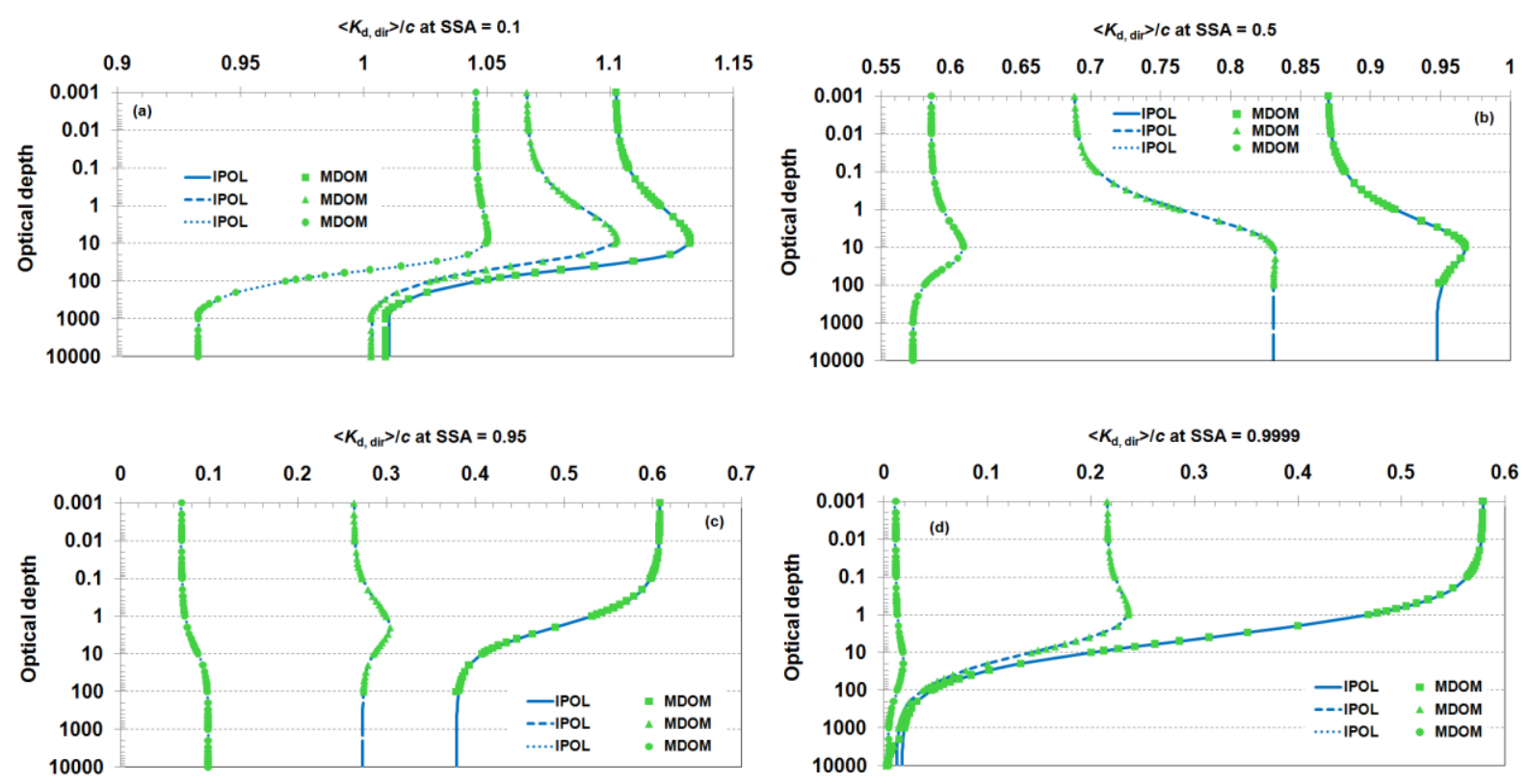

Fig. 5. A comparison of the IPOL (blue curves) and MDOM (green symbols) numerical computations for $\bar{K}_{\mathrm{d} \text {, dir }}(0-\rightarrow \tau) / c$. Computations were performed at the $\theta_{\mathrm{i}}=30^{\circ}$ for three $p(\theta)$ : with $g=0.002$ (solid curves and squares), 0.503 (dash curves and triangles), and 0.958 (dot curves and circles) and for four values of $\omega_{0}$ : (a) 0.1 , (b) 0.5 , (c) 0.95, and (d) 0.9999 .

Another criterion of estimation of numerical methods is checking of well-known physical constraints and boundary conditions. Sokoletsky et al. (2014a) and Sokoletsky and Budak (2016a, b) considered them in detail. Here we show a performance of only two physical constraints: 1) that following from the classical Bouguer-Lambert-Beer (BLB) law (Bouguer, 1729; Lambert, 1760; Beer, 1852) at $\omega_{0}=0$ (no scattering) and 2) the relationship (equality) between the deep regime parameter ("diffusion exponent") $k\left(g, \omega_{0}\right)$ and $\left[\bar{K}_{\mathrm{d}}(0-\rightarrow \tau) / c\right]\left(g, \omega_{0}\right)$ at $\tau \rightarrow \infty$ (McCormick, 1995; Haltrin, 1998; Mobley, 1994, 2017). 
The first physical constraint is

$$
\left.T(\tau)\right|_{\omega_{0}=0}=\exp \left[-\left(1-\omega_{0}\right) \tau_{\mathrm{ef}}\right]_{\omega_{0}=0}=\exp \left(-\tau_{\mathrm{ef}}\right) ; \bar{K}_{\mathrm{d}}(0-\rightarrow \tau) /\left.c\right|_{\omega_{0}=0}=1 / \bar{\mu}_{\mathrm{d}}
$$

and the second one is

$$
\left.\left[\bar{K}_{\mathrm{d}}(0-\rightarrow \tau) / c\right]\left(g, \omega_{0}\right)\right|_{\tau \rightarrow \infty}=k\left(g, \omega_{0}\right)
$$

We used $\omega_{0}=0.0001$ and $\tau=\tau_{\max }$ (a maximum of optical depth in the range of $0-<$ $\tau \leq 10000$, at which $T(\tau)>0$ ) as the reasonable approximations to $\omega_{0}=0$ and $\tau \rightarrow \infty$, respectively. Computation details for the diffusion exponent $k$ are described in Appendix A. As expected, calculations show practically coinciding results for IPOL and BLB at $\omega_{0}$ $=0.0001$ with an average difference of $0.02 \%$ at any $p(\theta)$; Fig. 6 is shown here as an example of such coincidence obtained for $p(\theta) \# 2$ from Table 2.

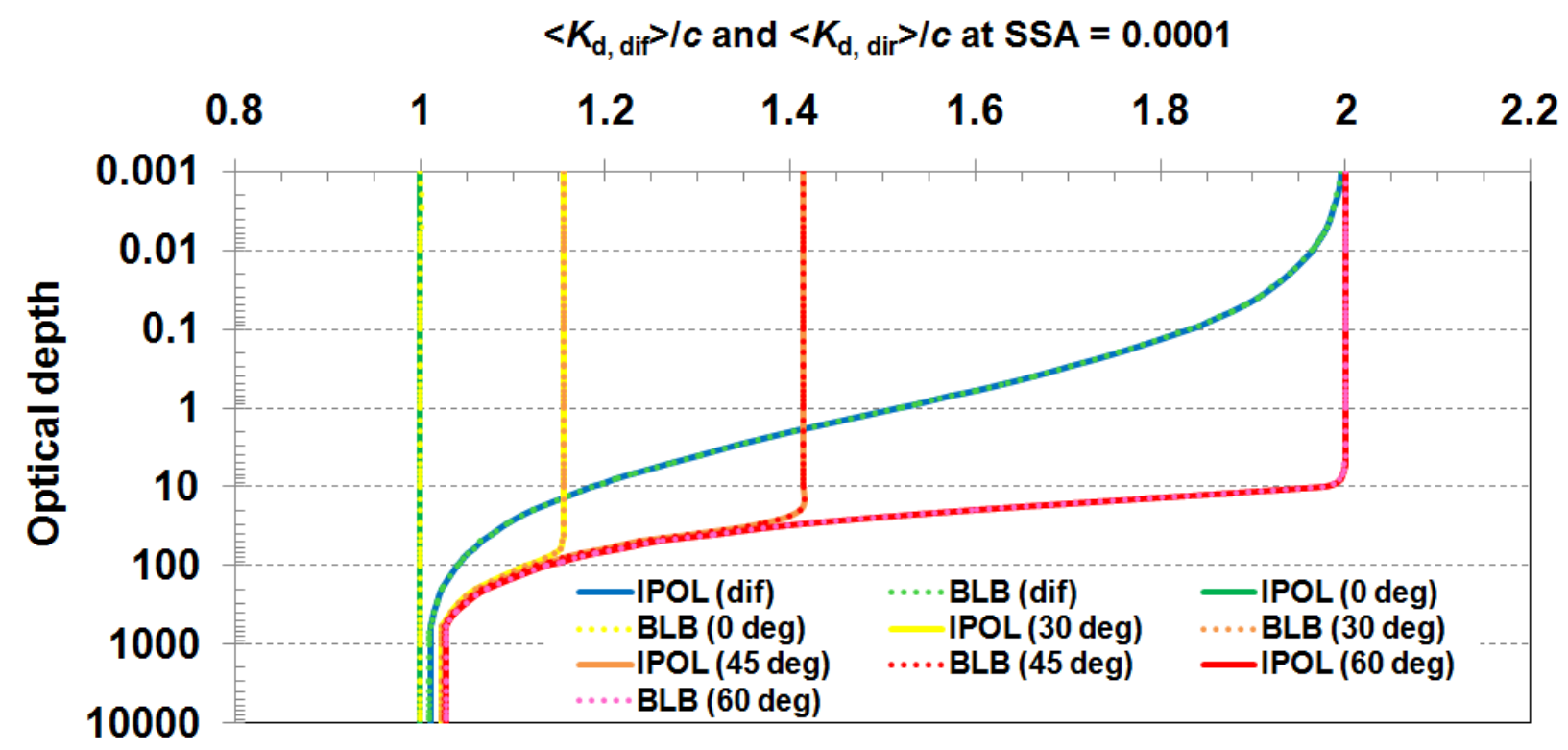

Fig. 6. A comparison of $\bar{K}_{\mathrm{d} \text {,dif }}(0-\rightarrow \tau) / c$ and $\bar{K}_{\mathrm{d} \text {, dir }}(0-\rightarrow \tau) / c$ calculated by the IPOL computations (solid curves) and BLB law (dotted curves) at $\omega_{0}=0.0001$. Computations 
were performed for diffuse and direct (at incident angles of $0^{\circ}, 30^{\circ}, 45^{\circ}$, and $60^{\circ}$ as shown in the legend) illumination and $p(\theta) \# 2$.

Similarly, a testing of Eq. (13) shows excellent results for almost all illumination geometries and optical conditions, excluding situations with $\omega_{0}=0.0001$ or $\omega_{0}=0.9999$ (Fig. 7), for which relative errors of up to $9 \%$ were found. Excluding these situations, a maximum of errors was about $1 \%$ with an average error of $0.5 \%$. For very small values of $\omega 0$, computer calculations are most likely to be affected by a loss of accuracy at high values of $\tau$ when transmittances become very close to zero (even at calculations by IPOL with 8 digits!); while for very large values of $\omega_{0}$, the main reason for discrepancies between $\left[\bar{K}_{\mathrm{d}}(0-\rightarrow \tau) / c\right]_{\tau=10000}$ and $k$ is that the former quantity is distinct from $\left[\bar{K}_{\mathrm{d}}(0-\rightarrow \tau) / c\right]_{\tau \rightarrow \infty}$ (even at $\left.\tau=10000 !\right)$, and no final value has yet been achieved for it with a reasonable accuracy.

After all, we selected the IPOL method as the benchmark for development and validation of analytical approximations for transmittances and albedos. All calculations of these optical properties were carried out with 5 or 8 digits to provide a high accuracy of $\bar{K}_{\mathrm{d}}(0-\rightarrow \tau) / c$. A high accuracy of calculation of $T(\tau)$ is especially important near the upper boundary of the layer, i.e., at low values of $\tau$ when calculation of $\bar{K}_{\mathrm{d}}(0-\rightarrow \tau) / c$ by Eq. (7) becomes unstable because the right part of this equation is a ratio of two quantities close to 0 in this case. 

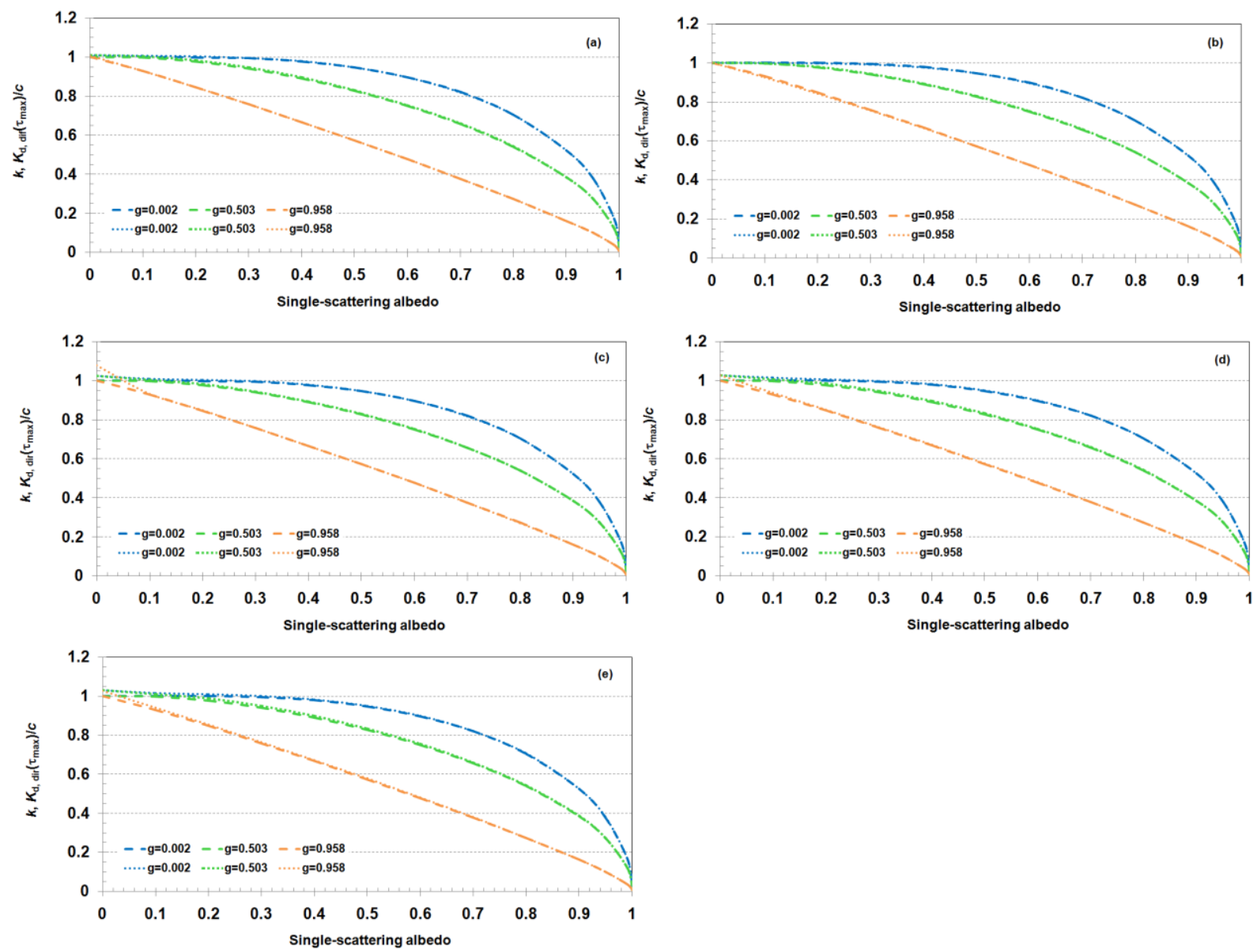

Fig. 7. A comparison of $\bar{K}_{\mathrm{d} \text {,dif }}\left(0-\rightarrow \tau_{\max }\right) / c$ and $\bar{K}_{\mathrm{d} \text {,dir }}\left(0-\rightarrow \tau_{\max }\right) / c$ calculated by the IPOL method (dotted curves) with the diffusion exponent $k$ calculated by Eqs. (A.1) and (A.2) (dashed curves, almost coinciding with the dotted curves) for: (a) diffuse illumination; (b) $\theta_{i}=0^{\circ}$; (c) $\theta_{i}=30^{\circ}$; (d) $\theta_{i}=60^{\circ}$; and (e) $\theta_{i}=89.99^{\circ}$ and three phase functions shown in the legends.

\subsection{Analytical methods}

We will consider two groups of analytical methods: 1) methods related to the GurevichKubelka-Munk (GKM) two-flux approximation to the radiative transfer and 2) methods non-related to the GKM approximation. 


\subsubsection{Methods related to the Gurevich-Kubelka-Munk (GKM) two-flux approximation}

\subsubsection{General considerations}

The authors of four pioneering publications, published in 1930s-50s were able to solve RTE for reflected and transmitted fluxes assuming only two fluxes: one traveling from the illuminated sample surface, and the other traveling toward the illuminated surface (Gurevič, 1930; Kubelka and Munk, 1931; Kubelka, 1948, 1954). This solution is therefore referred to as the Gurevich-Kubelka-Munk (GKM), or more often, the KubelkaMunk (KM) solution. However, the first scientists to suggest a two-flux solution of RTE were almost certainly Schuster (1905) and Schwarzschild (1906), which were probably unknown to the developers of the GKM.

A number of researchers following Kubelka (1948) (e.g., Sobolev, 1956; Hecht, 1976; Kokhanovsky, 2002; Barun and Ivanov, 2011) provided a relationship between $T(\tau)$ and two optical properties (IOPs), namely, albedo (reflectance) of infinite layer $R_{\infty}$ and diffusion exponent $k$ in the following form:

$$
T(\tau)=\frac{\left(1-R_{\infty}^{2}\right) \exp (-k \tau)}{1-R_{\infty}^{2} \exp (-2 k \tau)}
$$

where $k$ is completely determined by two ratios: $K / c$ and $S / c$ ( $K$ and $S$ are the GKM coefficients of absorption and scattering, respectively) as follows:

$$
k=\sqrt{\frac{K}{c}\left(\frac{K}{c}+\frac{2 S}{c}\right)} .
$$

Kokhanovsky (2007) has shown that Eq. (14) may be derived from the asymptotic radiative transfer under conditions of a black (non-reflecting) underlying surface. 
Moreover, Eq. (14) may be further generalized by taking into account the geometry of incident light. Assuming that the path length of the light beam per unit vertical distance is determined by the reciprocal of the average cosine for the downwelling irradiance $\left(\bar{\mu}_{\mathrm{d}}\right)^{-1}$ (Gordon, 1989; Kirk, 1991, 1999; Sathyendranath and Platt, 1997; see also our Fig. 1), an expression for the transmitted light may be rewritten in the form:

$$
T(\tau)=\frac{\left(1-R_{\infty}^{2}\right) \exp \left(-k \tau_{\mathrm{ef}}\right)}{1-R_{\infty}^{2} \exp \left(-2 k \tau_{\mathrm{ef}}\right)}
$$

where $\tau_{\mathrm{ef}}=\tau / \bar{\mu}_{\mathrm{d}}(\tau)$ is the effective optical depth (thickness). Generally speaking, Eqs.

(14) and (16) give the same results only at $\bar{\mu}_{d}=1$, which is in turn possible only for very special cases, for example, (a) at normal incidence of light (i.e., at $\theta_{i}=0^{\circ}$ ) on the interface between the outer and inner layers, with the additional condition of complete light absorption (i.e., $\omega_{0}=0$ ) or (b) far away from the interface, in the asymptotic light regime, under any geometrical and optical conditions. Therefore, we subsequently use only Eq. (16) for investigation of transmitted light.

Note that equations for transmittance such as Eqs. (14) and (16) may be found in numerous publications, e.g., by Stokes (1862), Ambartsumian (1942), Tuckerman (1947), Sobolev (1957), Ueno (1961), Hébert and Machizaud (2012), Sokoletsky et al. (2014a), and Sokoletsky and Budak (2016a, b, 2017). However, to the best of our knowledge, a presentation of $\tau_{\mathrm{ef}}$ in the form of $\tau_{\mathrm{ef}}=\tau / \bar{\mu}_{\mathrm{d}}(\tau)$ was considered in only few publications, e.g., by Ambartsumian (1942), Sobolev (1957), Ueno (1961), Carder et al. (1999), Hébert and Machizaud (2012), Sokoletsky et al. (2014a), and Sokoletsky and Budak (2016a, b, 2017). In the other publications, instead of $\bar{\mu}_{d}(\tau)$, cosine $\mu_{i}$ has been used, which may be inaccurate, especially far from the top surface and at strong 
scattering (see, e.g., Fig. 3 of Morel and Loisel, 1998 and Fig. 3 of Aas and Højerslev, 1999). Sokoletsky and Budak (2016b, 2017) extended a parameterization of $\bar{\mu}_{d}(\tau)$ for a diffuse incident radiation, keeping, however, a simplified parameterization for direct light.

\subsubsection{Approximations for $k, \bar{\mu}_{\mathrm{d}}(\tau), \boldsymbol{r}_{\infty}$, and $R_{\mathrm{p} \infty}$}

Based on the previous (see Table 1) and present results, we will limit ourselves to consideration of the well-known approximations and those that have been proven to be accurate and reliable compared with the numerical solutions of RTE. A mathematical apparatus for approximated calculations of diffusion exponent $k$, average cosine for the downwelling irradiance $\bar{\mu}_{\mathrm{d}}(\tau)$, spherical albedo of semi-infinite or infinite layer $r_{\infty}$, and plane albedo of semi-infinite or infinite layer $R_{p \infty}$ is described in Appendices $A, B, C$, and D, respectively.

\subsubsection{Sensitivity analysis of $\bar{K}_{d}(0-\rightarrow \tau) / c$ vs. $\bar{\mu}_{d}(\tau)$}

In order to estimate the sensitivity of the estimates of $\bar{K}_{\mathrm{d}}(0-\rightarrow \tau) / c$ to errors produced by $\bar{\mu}_{\mathrm{d}}(\tau)$ and to predict the relative errors $\delta\left[\bar{K}_{\mathrm{d}}(0-\rightarrow \tau) / c\right]$, we calculated first the error amplification factor ("normalized sensitivity coefficient" is another its name) $\Phi\left[\bar{K}_{\mathrm{d}}(0-\rightarrow \tau) / c\right.$ vs. $\left.\bar{\mu}_{\mathrm{d}}(\tau)\right]=\delta\left[\bar{K}_{\mathrm{d}}(0-\rightarrow \tau) / c\right] / \delta \bar{\mu}_{\mathrm{d}}(\tau)=\left\{\bar{\mu}_{\mathrm{d}}(\tau) /\left[\bar{K}_{\mathrm{d}}(0-\rightarrow \tau) / c\right]\right\}$ $\times\left\{\partial\left[\bar{K}_{\mathrm{d}}(0-\rightarrow \tau) / c\right] / \partial \bar{\mu}_{\mathrm{d}}(\tau)\right\}$ (see, e.g., Shifrin, 1988, p. 212; Leathers and McCormic, 1997) (Fig. 8a) and then multiplied these values on the relative errors $\delta \bar{\mu}_{d}(\tau)$ to obtain the predicted values of $\delta\left[\bar{K}_{\mathrm{d}}(0-\rightarrow \tau) / c\right]$ (Fig. 8b). Results show that predicted level of 
relative errors in $\bar{K}_{\mathrm{d}}(0-\rightarrow \tau) / c$ is generally smaller than in $\bar{\mu}_{\mathrm{d}}(\tau) \quad$ i.e., $\Phi\left[\bar{K}_{\mathrm{d}}(0-\rightarrow \tau) / c\right.$ vs. $\left.\left.\bar{\mu}_{\mathrm{d}}(\tau)\right]<1\right\}$ and, hence, they are expected to be $<10 \%$ at $\theta \mathrm{i}<45^{\circ}$ under assumption that the values of $\tau, k$, and $R_{\infty}$ are exact.
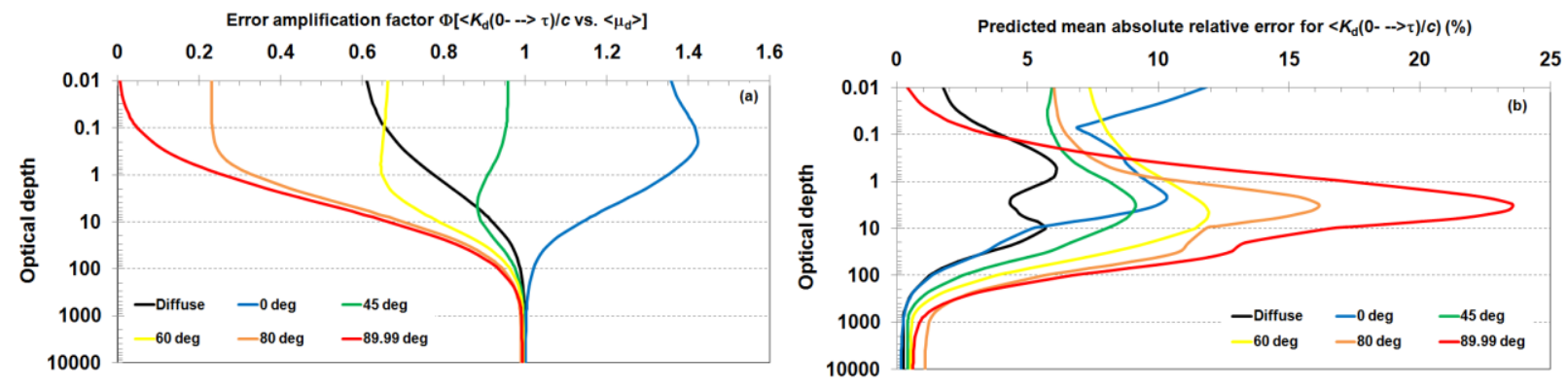

Fig. 8. An analysis of sensitivity and accuracy for $\bar{K}_{\mathrm{d}}(0-\rightarrow \tau) / c$ vs. errors in $\bar{\mu}_{\mathrm{d}}(\tau)$ :

error amplification factor $\Phi\left[\bar{K}_{\mathrm{d}}(0-\rightarrow \tau) / c\right.$ vs. $\left.\bar{\mu}_{\mathrm{d}}(\tau)\right](\mathrm{a})$; and predicted mean absolute relative error of $\bar{K}_{\mathrm{d}}(0-\rightarrow \tau) / c \quad(\mathrm{~b})$ at selected illumination conditions (in the legends), $p(\theta) \# 1$ to 3 from Table 2, and averaged for 14 selected $\omega_{0}$.

\subsubsection{Methods unrelated to the GKM approximation}

We will consider here several models unrelated to GKM theory. All models refer to spherical transmittance $t(\tau)$ or plane transmittance $T_{\mathrm{p}}(\mu \mathrm{i}, \tau)$, or may be used for both cases [with the corresponding parameterization of $\bar{\mu}_{d}(\tau)$ ]. As mentioned above, we will replace an optical depth (or thickness) $\tau$ by its effective value of $\tau_{\mathrm{ef}}=\tau / \bar{\mu}_{\mathrm{d}}(\tau)$ in all models even though it was not assumed in the original model. Such replacement allows for the strong increasing of the range of applicability of the models, making them more accurate and robust. 


\subsubsection{Approximations applied for both $t(\tau)$ and $T_{\mathrm{p}}(\mu \mathrm{i}, \tau)$}

We will consider here analytical models that may be applied for the both $t(\tau)$ and $T_{\mathrm{p}}(\mu \mathrm{i}$, $\tau):$

(a) A classical BLB model (Bouguer, 1729; Lambert, 1760; Beer, 1852):

$$
T(\tau)=\exp \left(-a z / \bar{\mu}_{\mathrm{d}}\right)=\exp \left[-\left(1-\omega_{0}\right) \tau_{\mathrm{ef}}\right] .
$$

(b) A quasi-single-scattering approximation, QSSA (Gordon et al., 1975): Eq. (6) and

$$
\frac{\bar{K}_{\mathrm{d}}(0-\rightarrow \tau)}{c}=\frac{1-\omega_{0} F}{\bar{\mu}_{\mathrm{d}}(\tau)} .
$$

(c) Hulst (1980, Ch. 14) and Ben-David (1995, 1997) model:

$$
T(\tau)=\frac{z}{x \sinh \left(z \tau_{\mathrm{ef}}\right)+z \cosh \left(z \tau_{\mathrm{ef}}\right)}, x=1-0.5(1+g) \omega_{0}, z=\sqrt{\left(1-\omega_{0}\right)\left(1-g \omega_{0}\right)} .
$$

(d) Aas (1987) model: Eq. (6) and

$$
\frac{\bar{K}_{\mathrm{d}}(0-\rightarrow \tau)}{c}=\frac{1-0.978 \omega_{0}}{\bar{\mu}_{\mathrm{d}}(\tau)} .
$$

(e) Gordon (1989) approximation: Eq. (6) and

$$
\frac{\bar{K}_{\mathrm{d}}(0-\rightarrow \tau)}{c}=\frac{1.04\left(1-\omega_{0} F\right)}{\bar{\mu}_{\mathrm{d}}(\tau)} .
$$

(f) Kirk's (1991) approximation: Eq. (6) and

$$
\frac{\bar{K}_{\mathrm{d}}(0-\rightarrow \tau)}{c}=\frac{\left(1-\omega_{0}\right) \sqrt{1+\left(A \bar{\mu}_{\mathrm{d}}(\tau)-B\right) \omega_{0} /\left(1-\omega_{0}\right)}}{\bar{\mu}_{\mathrm{d}}(\tau)},
$$

where $A=2.636 / g-2.447$ and $B=0.849 / g-0.739$.

(g) Cornet et al. (1992) model: 


$$
T(\tau)=\frac{4 k}{(1+k)^{2} \exp \left(k \tau_{\mathrm{ef}}\right)-(1-k)^{2} \exp \left(-k \tau_{\mathrm{ef}}\right)} .
$$

(h) Haltrin (1998) model:

$$
\begin{gathered}
T(\tau)=\frac{\left[1+R_{0} \xi(\tau)\right] \exp \left(-k \tau_{\mathrm{ef}}\right)}{1+R_{0} \xi(\tau) \exp \left(-k \tau_{\mathrm{ef}}\right)}, R_{0}=\left(\frac{2+\bar{\mu}_{\infty}}{2-\bar{\mu}_{\infty}}\right) R_{\infty}, \quad R_{\infty}=\left(\frac{1-\bar{\mu}_{\infty}}{1+\bar{\mu}_{\infty}}\right)^{2}, \\
\bar{\mu}_{\infty}=\sqrt{\frac{1+2 G-\sqrt{G(4+5 G)}}{1+G}}, G=\frac{\omega_{0} B}{1-\omega_{0} F}, \quad \xi(\tau)=-R_{\infty} \exp \left(-\frac{v}{c} \tau_{\mathrm{ef}}\right), \\
\frac{v}{c}=\frac{7+2 \bar{\mu}_{\infty}^{2}-\bar{\mu}_{\infty}^{4}}{3-\bar{\mu}_{\infty}^{2}} k .
\end{gathered}
$$

(i) Lee et al. (2005a, b) model: Eq. (6) and

$$
\begin{gathered}
\frac{\bar{K}_{\mathrm{d}}(0-\rightarrow \tau)}{c}=\frac{\bar{K}_{\mathrm{d}}\left(0-\rightarrow \tau_{\mathrm{m}}\right)}{c} \frac{\bar{\mu}_{\mathrm{d}}\left(\tau_{\mathrm{m}}\right)}{\bar{\mu}_{\mathrm{d}}(\tau)}, \tau_{\mathrm{m}}=\frac{\ln 10}{\bar{K}_{\mathrm{d}}\left(0-\rightarrow \tau_{\mathrm{m}}\right) / c}, \\
\frac{\bar{K}_{\mathrm{d}}\left(0-\rightarrow \tau_{\mathrm{m}}\right)}{c}=\left(1+0.005 \theta_{0}\right)\left(1-\omega_{0}\right)+3.47 \omega_{0} B, \\
\theta_{0}=\left\{\begin{array}{l}
45^{\circ} \text { for } t(\tau) \\
\arcsin \left(1.340 \theta_{\mathrm{i}}\right) \text { for } T_{\mathrm{p}}(\tau)
\end{array} .\right.
\end{gathered}
$$

(j) Pottier et al. (2005) model:

$$
T(\tau)=\frac{4 \alpha}{(1+\alpha)^{2} \exp \left(k \tau_{\mathrm{ef}}\right)-(1-\alpha)^{2} \exp \left(-k \tau_{\mathrm{ef}}\right)}, \quad \alpha=\sqrt{\frac{1-\omega_{0}}{1-\omega_{0}+2 \omega_{0} B}}
$$

Note the mathematical equivalence between Eqs. (30) and the GKM theory for $t(\tau)$ expressed by Eqs. (16) and (C.2) under the assumption that $K / S=a / b_{b}$; however, we used here a diffusion exponent $k$ computed differently (see Appendix A) from that used in GKM.

\subsubsection{Approximations applied for $t(\tau)$ only}


(a) Kokhanovsky and Hopkinson (2008) model:

$$
t(\tau)=\frac{\exp \left(-k \tau_{\mathrm{ef}}\right)-\exp \left(-k \tau_{\mathrm{ef}}-8 s / \sqrt{3}\right)}{1-\exp \left(-2 k \tau_{\mathrm{ef}}-8 s / \sqrt{3}\right)},
$$

where similarity parameter $s$ is defined by Eq. (A.5).

(b) Pan and Zimmerman (2010) model: Eq. (6) and

$$
\begin{aligned}
\frac{\bar{K}_{\mathrm{d}, \mathrm{dif}}(0-\rightarrow \tau)}{c} & =\frac{\bar{\mu}_{\mathrm{d}, \mathrm{dif}}(0-)}{\bar{\mu}_{\mathrm{d}, \mathrm{dif}}(\tau)}\left[1.317-(1.399-1.012 \sqrt{B}-0.939 B) \omega_{0}\right. \\
& \left.+(0.047-0.244 \sqrt{B}-1.120 B) \omega_{0}^{2}\right]
\end{aligned}
$$

\subsubsection{Approximations applied for $T_{p}(\tau)$ only}

(a) The Nechad and Ruddick (2010) model has been used for plane transmittance only: Eq. (6) and

$$
\begin{aligned}
\frac{\bar{K}_{\mathrm{d}, \mathrm{dir}}(0-\rightarrow \tau)}{c} & =\left(0.53+0.49 \cosh \theta_{0}\right)\left(1-\omega_{0}\right)+\omega_{0} B\left(9.776-4.56 \cosh \theta_{0}\right) \\
& \times\left(0.53+0.49 \cosh \theta_{0}\right)^{2}\left(1-\frac{\omega_{0} B}{1-\omega_{0}}\right), \theta_{0}=\arcsin \left(1.340 \theta_{\mathrm{i}}\right) .
\end{aligned}
$$

(b) Pan and Zimmerman (2010) model: Eq. (6) and

$$
\begin{aligned}
& \frac{\bar{K}_{\mathrm{d}, \mathrm{dir}}(0-\rightarrow \tau)}{c}=\frac{1}{\bar{\mu}_{\mathrm{d}, \mathrm{dir}}(\tau)}\left[k+\frac{\left(1-\omega_{0} F-k\right)\left(1-e^{-P \tau}\right)}{P \tau}\right], \\
& P=0.817-0.877 \sqrt{\omega_{0}}+\left(0.193+0.421 \omega_{0}+0.741 \omega_{0}^{2}\right) \sqrt{B} .
\end{aligned}
$$

\section{Results for transmittances and diffuse attenuation coefficients}

4.1 Results for $\bar{K}_{\mathrm{d} \text {, dif }}(0-\rightarrow \tau)$ 
To reveal errors of analytical models unrelated to the errors of $\bar{\mu}_{\mathrm{d} \text {, dif }}(\tau)$, we considered first (training step) all models using $\bar{\mu}_{\mathrm{d} \text {,dif }}(\tau)$ following from the numerical (IPOL) computations, expressed by Eq. (B.1). We compared the analytical solutions with numerical solutions for given $p(\theta) \# 1-3$ and selected values of $\omega_{0}$ at optical depths $\tau$ varied from 0.0001 to 10000 . The main statistical measure used for the model estimation was the normalized root-mean-square error calculated as

$$
\operatorname{NRMSE}(\%)=100 \% \frac{\sqrt{\sum_{i=1}^{n} \frac{\left(\tilde{x}_{\mathrm{i}}-x_{\mathrm{i}}\right)^{2}}{n-1}}}{\bar{x}},
$$

where $\tilde{x}_{\mathrm{i}}$ and $x_{\mathrm{i}}$ are the analytical (approximated) and numerical (accepted as a reference) values of $\bar{K}_{\mathrm{d} \text {, dif }}(0-\rightarrow \tau)$, respectively; $\bar{x}$ is the averaged value for the given set of $x_{\mathrm{i}} ; n$ is the number of couples $\tilde{x}_{\mathrm{i}}$ and $x_{\mathrm{i}}$ under comparison (this number is determined by a number of optical depths considered in this study, i.e., $n=64$ ).

Table 3 shows results for the models outlined above at three $p(\theta)(\# 1-3)$ and five values of $\omega_{0}$. All models were estimated by their NRMSE (Eq. 36); if it was necessary, the spherical transmittance was converted to the $\bar{K}_{\mathrm{d} \text {, dif }}(0-\rightarrow \tau) / c$ by Eq. (7).

Table 3. Normalized (to the average) root-mean-square errors (\%) of analytically computed $\bar{K}_{\mathrm{d} \text {, dif }}(0-\rightarrow \tau)$ relative to corresponding values of $\bar{K}_{\mathrm{d} \text {, dif }}(0-\rightarrow \tau)$ computed by the IPOL method for 64 optical depths at five selected values of $\omega_{0}$ (columns 3-7), three different $p(\theta)$ (left to right, top to bottom, in order, corresponds to 
$p(\theta) \# 1-3$ in Table 2$)$, and $\bar{\mu}_{\mathrm{d} \text {,dif }}(\tau)$ derived from the IPOL-based values of $R_{\infty}$ and $\left.T(\tau)\right]$, Eq. (B.1).

\begin{tabular}{|c|c|c|c|c|c|c|}
\hline$\# / \omega_{0}$ & $\begin{array}{c}\text { Model } \\
\text { equations }\end{array}$ & 0.0001 & 0.1 & 0.5 & 0.95 & 0.9999 \\
\hline 1 & $\begin{array}{c}\text { 16, A.1-A.3, } \\
\text { B.1, C.1 }\end{array}$ & $\begin{array}{l}4.2 \times 10^{-5} \\
4.2 \times 10^{-5} \\
4.1 \times 10^{-5}\end{array}$ & $\begin{array}{c}0.030 \\
0.0054 \\
8.0 \times 10^{-5}\end{array}$ & $\begin{array}{c}1.1,0.30 \\
0.0063\end{array}$ & $11,6.3,1.6$ & $20,11,31$ \\
\hline 2 & $\begin{array}{l}\text { 16, A.1-A.3, } \\
\text { A.5, B.1, C.3 }\end{array}$ & $\begin{array}{c}1.5,0.21 \\
4.1 \times 10^{-5}\end{array}$ & $\begin{array}{c}1.8,0.28 \\
1.1 \times 10^{-4}\end{array}$ & $\begin{array}{c}2.3,0.43, \\
0.0091\end{array}$ & $\begin{array}{c}0.087 \\
0.88,1.4\end{array}$ & $\begin{array}{c}0.10,0.14 \\
0.53\end{array}$ \\
\hline 3 & $\begin{array}{c}\text { 15, 16, A.4, } \\
\text { A.6, B.1, C.3, } \\
\text { C.5, C. } 20 \\
\text { C. } 21\end{array}$ & $\begin{array}{c}1.5,0.21 \\
0.017\end{array}$ & $1.8,2.7,2.1$ & $\begin{array}{l}3.1,5.1 \\
\quad 3.8\end{array}$ & $\begin{array}{c}0.068,1.7 \\
1.5\end{array}$ & $\begin{array}{c}0.30,0.30 \\
1.0\end{array}$ \\
\hline 4 & $\begin{array}{l}\text { 16, A.1-A.3, } \\
\text { B.1, C.4, C.5 }\end{array}$ & $\begin{array}{l}4.2 \times 10^{-5} \\
4.2 \times 10^{-5} \\
4.1 \times 10^{-5}\end{array}$ & $\begin{array}{c}0.012 \\
9.3 \times 10^{-4} \\
2.6 \times 10^{-5}\end{array}$ & $\begin{array}{c}0.35 \\
0.069 \\
6.6 \times 10^{-5}\end{array}$ & $\begin{array}{c}1.2,1.1 \\
0.17\end{array}$ & $\begin{array}{c}0.10,0.14, \\
0.47\end{array}$ \\
\hline 5 & $\begin{array}{c}15,16, \text { A.4- } \\
\text { A.6, B.1, C.4, } \\
\text { C.5, C. } 20 \\
\text { C. } 21\end{array}$ & $\begin{array}{c}1.3 \times 10^{-4} \\
0.0060 \\
0.017\end{array}$ & $\begin{array}{c}0.0094,2.4 \\
2.1\end{array}$ & $\begin{array}{c}0.65,4.6 \\
\quad 3.8\end{array}$ & $\begin{array}{c}1.1,1.9 \\
0.30\end{array}$ & $\begin{array}{c}0.30,0.30 \\
0.95\end{array}$ \\
\hline 6 & $\begin{array}{l}\text { 16, A.1-A.3, } \\
\text { A.5, B.1, C.7 }\end{array}$ & $\begin{array}{l}4.2 \times 10^{-5} \\
4.2 \times 10^{-5} \\
4.1 \times 10^{-5}\end{array}$ & $\begin{array}{l}0.0058 \\
8.3 \times 10^{-4} \\
3.0 \times 10^{-5}\end{array}$ & $\begin{array}{c}0.13 \\
0.017 \\
0.0010\end{array}$ & $\begin{array}{c}0.19 \\
0.049 \\
0.029\end{array}$ & $\begin{array}{l}0.019 \\
0.016 \\
0.021\end{array}$ \\
\hline 7 & $\begin{array}{c}\text { 15, 16, A.4- } \\
\text { A.6, C.5, C. } 7 \\
\text { C. } 20, \text { C. } 21\end{array}$ & $\begin{array}{c}1.3 \times 10^{-4} \\
0.0060 \\
0.017\end{array}$ & $\begin{array}{c}0.012,2.4 \\
2.1\end{array}$ & $\begin{array}{c}0.78,4.7 \\
3.8\end{array}$ & $\begin{array}{c}0.081 \\
0.90,0.12\end{array}$ & $\begin{array}{c}0.18,0.14 \\
0.51\end{array}$ \\
\hline 8 & $\begin{array}{l}\text { 16, A.1-A.3, } \\
\text { B.1, C.8, C.9 }\end{array}$ & $\begin{array}{l}5.3 \times 10^{-5} \\
5.4 \times 10^{-5} \\
5.3 \times 10^{-5}\end{array}$ & $\begin{array}{c}0.011 \\
0.010 \\
5.3 \times 10^{-5}\end{array}$ & $\begin{array}{c}0.079 \\
0.55 \\
0.0072\end{array}$ & $13,12,1.9$ & $\begin{array}{c}128,123 \\
94\end{array}$ \\
\hline 9 & $\begin{array}{c}\text { 16, A.1-A.3, } \\
\text { B.1, C.2, } \\
\text { C. } 10\end{array}$ & $\begin{array}{l}0.0037 \\
0.0037 \\
0.0037\end{array}$ & $\begin{array}{c}0.0068 \\
0.012 \\
0.0036\end{array}$ & $\begin{array}{c}1.6,0.84, \\
0.0053\end{array}$ & $\begin{array}{c}1.9,2.2 \\
1.5\end{array}$ & $\begin{array}{c}0.10,0.15 \\
0.52\end{array}$ \\
\hline 10 & $\begin{array}{c}\text { 15, 16, A.1- } \\
\text { A.3, B.1, C.2, } \\
\text { C.5, C. } 11 \\
\text { C. } 12\end{array}$ & $\begin{array}{l}0.023 \\
0.023 \\
0.023\end{array}$ & $\begin{array}{l}0.055 \\
0.048 \\
0.026\end{array}$ & $\begin{array}{l}0.41 \\
0.012 \\
0.045\end{array}$ & $\begin{array}{c}1.4,1.5 \\
0.18\end{array}$ & $\begin{array}{c}0.10,0.14 \\
0.49\end{array}$ \\
\hline 11 & $\begin{array}{c}\text { 15, 16, A.4, } \\
\text { A.6, B.1, C. } 2 \\
\text { C. } 5, \text { C. } 11 \\
\text { C. } 12, \text { C. } 20\end{array}$ & $\begin{array}{l}0.023 \\
0.018 \\
0.013\end{array}$ & $\begin{array}{c}0.068,2.5 \\
2.1\end{array}$ & $\begin{array}{c}0.62,4.7 \\
3.8\end{array}$ & $\begin{array}{c}1.3,2.2 \\
0.31\end{array}$ & $\begin{array}{c}0.30,0.30 \\
0.97\end{array}$ \\
\hline
\end{tabular}


C. 21

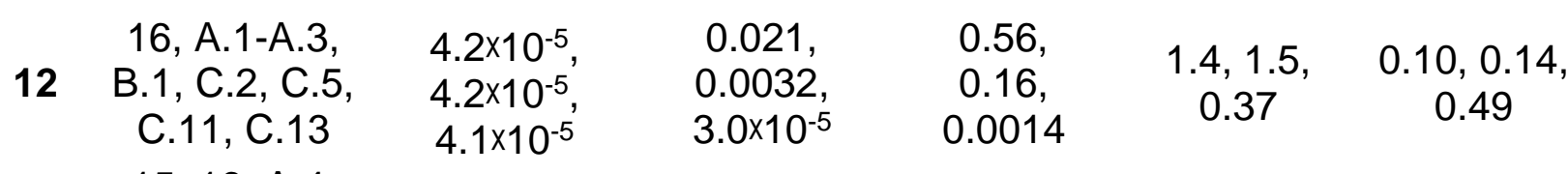
15, 16, A.4,

A.6, B.1, C. $2, \quad 1.3 \times 10^{-4}, \quad 0.012,2.4, \quad 0.57,4.6, \quad 1.3,2.3, \quad 0.30,0.30$,

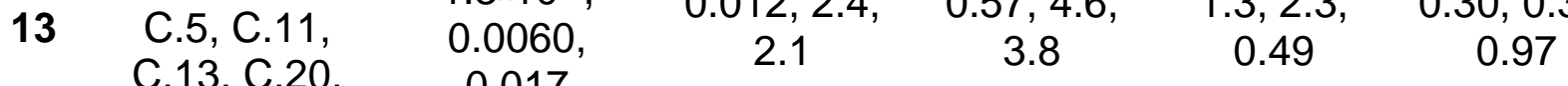
$\begin{array}{ll}\text { C. } 13, \text { C. } 20, & 0.017 \\ \text { C. } 21 & \end{array}$

14

16, A.1-A.3

B. 1,24, C. 14

$4.2 \times 10^{-5}$
$4.2 \times 10^{-5}$ $4.1 \times 10^{-5}$

0.023, $\quad 0.023$, 0.0037 , $3.2 \times 10^{-5}$

0.25 , 0.0017

$0.28,8.7$ 0.64

0.33, 25, $4.3 \times 10^{-5}$, 16, A.1-A.3, 1.8, 0.48, 1.8, 21, 0.0038 0.29

11, 1236, 48 15, 134, $4.1 \times 10^{-5}$

0.071 , 16, A.1-A.3, $4.1 \times 10^{-5}$,

16

B.1, C.16, C. 17 $4.2 \times 10^{-5}$, 16, A.1-A.3,

17 B.1, C.2, C.5,

C.11, C.18, $4.1 \times 10^{-5}$ 0.016 , $1.1 \times 10^{-4}$ $2.4,0.59$
0.0071 $8.3,6.4$
1.6 $20,11,31$ C. 19

15, 16, A.4,

18 A.6, B.1, C.2, C.5, C.11,

$\begin{array}{ccc}2.9,2.9,2.9 & 1.5,2.1,2.8 & 0.65 \\ & 0.27,2.3\end{array}$

2.1, 1.9, $0.10,0.14$, 0.49 2.8 C.18-C.21

$2.9,2.9,2.8 \quad 1.5,4.4,1.6$

$0.55,4.5$,

1.9, 2.7, 0.30, 0.30, 2.3 0.62 2.3 16, A.1-A.3,

19 16, A.1-A.3, $4.2 \times 10^{-5}$, $4.2 \times 10^{-5}$, C. 11, C. $20, \quad 4.1 \times 10^{-5}$ C. 21 15, 16, A.4- $1.3 \times 10^{-4}$, 20 A.6, B.1, C.5, 0.0060 , C.20, C.21 0.017

21 16, A.1-A.3, 0.0037 B.1, C.2, C.22 0.0037 15, 16, A.4,

22 A.6, B. 1, C.5, C.20-C.22 0.0037

$$
\begin{aligned}
& 0.030 \\
& 0.0059 \\
& 4.6 \times 10^{-5}
\end{aligned}
$$

0.71 , 0.25 , 0.0031 $\begin{array}{cc}1.4,1.4, & 0.28,0.32 \\ 0.52 & 0.64\end{array}$ 0.0036 , 0.0037 0.0015 $0.020,2.4, \quad 0.55,4.5$ 2.1 3.8

$1.3,2.2$ 0.65 $0.48,0.48$, 1.1 0.068 0.012 , 0.0036 1.6, 0.95, 1.9, 3.9, $\begin{array}{lll}0.0053 & 2.4 & 0.66\end{array}$ $0.10,0.15$ $0.056,2.4, \quad 1.1,4.0, \quad 1.7,4.6, \quad 0.30,0.31$ 2.1 3.8 2.5 1.1

$\begin{array}{ll}\text { B.1, } 17 & 0.010, \\ & 0.026, \\ & 0.024\end{array}$

$10,10,3.4 \quad 50,41,13$

$101,94,53$

136,134 125 


\begin{tabular}{|c|c|c|c|c|c|c|}
\hline 24 & 1,18 & $\begin{array}{c}0.0052 \\
0.024 \\
0.024\end{array}$ & $5.3,8.5,3.3$ & $23,31,12$ & $35,54,44$ & $82,81,90$ \\
\hline & B. 1,19 & $\begin{array}{c}0.0052 \\
0.023 \\
0.024\end{array}$ & $5.3,7.6,3.1$ & $25,26,11$ & $38,38,33$ & $44,43,42$ \\
\hline & B. 1,20 & $\begin{array}{l}0.010 \\
0.025 \\
0.024\end{array}$ & $10,9.9,3.1$ & $49,40,11$ & $98,88,31$ & $\begin{array}{c}132,125 \\
42\end{array}$ \\
\hline 7 & B. 1,21 & $4.2,4.2,4.2$ & $\begin{array}{c}1.3,4.7 \\
0.77\end{array}$ & $\begin{array}{c}20,29 \\
8.8\end{array}$ & $34,52,42$ & $83,79,88$ \\
\hline & B.1, 22 & $\begin{array}{l}2.7,0.021 \\
\quad 0.024\end{array}$ & $\begin{array}{c}601,5.7 \\
3.1\end{array}$ & $\begin{array}{c}1270,13 \\
11\end{array}$ & $976,46,31$ & $\begin{array}{c}95,130 \\
58\end{array}$ \\
\hline & $\begin{array}{c}\text { 23, A.1-A.3 } \\
\text { B. } 1\end{array}$ & $\begin{array}{l}4.1 \times 10^{-5}, \\
4.3 \times 10^{-5} \\
4.2 \times 10^{-5}\end{array}$ & $\begin{array}{r}0.07 \\
0.016\end{array}$ & $\begin{array}{c}2.9,0.32 \\
12\end{array}$ & $27,20,318$ & $\begin{array}{c}43,43 \\
1708\end{array}$ \\
\hline 0 & $\begin{array}{c}\text { 24-26, A.1- } \\
\text { A.3, B.1 }\end{array}$ & $\begin{array}{l}4.2 \times 10^{-5} \\
4.2 \times 10^{-5} \\
4.1 \times 10^{-5}\end{array}$ & $\begin{array}{c}0.053 \\
0.0059 \\
7.7 \times 10^{-5}\end{array}$ & $\begin{array}{c}1.7,0.32 \\
0.0059\end{array}$ & $18,7.2,1.4$ & $39,17,22$ \\
\hline 31 & 27-29, B.1 & A, 14, 14 & 7.7, & $\begin{array}{c}29,6.7 \\
15\end{array}$ & $\begin{array}{c}183,57 \\
764\end{array}$ & $\begin{array}{c}315,139 \\
1145\end{array}$ \\
\hline 32 & $\begin{array}{c}\text { 30, A.1-A.3 } \\
\text { B.1 }\end{array}$ & $\begin{array}{l}4.2 \times 10^{-5} \\
4.2 \times 10^{-5} \\
4.1 \times 10^{-5}\end{array}$ & $\begin{array}{l}0.030 \\
0.0054 \\
8.0 \times 10^{-5}\end{array}$ & $\begin{array}{c}1.1,0.30 \\
0.0063\end{array}$ & $11,6.3,1.6$ & $20,11,31$ \\
\hline 33 & $\begin{array}{c}\text { 31, A.1-A.3 } \\
\text { A.5, B.1 }\end{array}$ & $1.5,1.5,1.5$ & $1.8,1.6,1.5$ & $\begin{array}{c}2.3,2.3 \\
1.7\end{array}$ & $\begin{array}{c}0.083 \\
0.74,3.0\end{array}$ & $\begin{array}{c}0.10,0.13 \\
0.39\end{array}$ \\
\hline 34 & $\begin{array}{c}15,31, \text { A.4- } \\
\text { A.6, B.1, C.5, } \\
\text { C. } 20, \text { C. } 21\end{array}$ & $1.5,1.5,1.5$ & $1.8,3.9,1.2$ & $\begin{array}{c}3.1,6.8 \\
2.6\end{array}$ & $\begin{array}{c}0.070 \\
0.36,2.9\end{array}$ & $\begin{array}{c}0.30,0.29 \\
0.87\end{array}$ \\
\hline & 32, B. 1 & $36,36,36$ & $33,35,36$ & $29,36,37$ & $57,54,48$ & $85,83,76$ \\
\hline
\end{tabular}

Table 3 demonstrates that 19 models (\#2-7, 9-13, 17-22, 33, and 34) among 35 show a high accuracy (better than $6.8 \%$ ) at all values of optical parameters. 34 models (all, excluding 35) give excellent results at wo close to 0 , while model \#35 [Pan and Zimmerman (2010)] yields relatively weak results at all $\omega_{0}$.

Interestingly, there is no strong connection between performance of $r_{\infty}$ and $\bar{K}_{\mathrm{d}, \mathrm{dif}}(0-\rightarrow \tau)$ for all models. While four models, GKM+Mudgett and Richards (1971, 
1972), Hulst's (1974, 1980), GKM+van Gemert and Star (1987), and GKM+Sokoletsky et al. (2013), show excellent results for both these optical properties, HKS 1 demonstrated excellent results (relative logarithmic errors up to $4 \%$, see Fig. C.2j) only for $r_{\infty}$, but results for $\bar{K}_{\mathrm{d} \text {, dif }}(0-\rightarrow \tau) \quad\left(\right.$ at $\left.\omega_{0} \geq 0.95\right)$ were less successful. In contrast, the Rozenberg, GKM+modified $\delta-E(2), G K M+$ modified $\delta-E(4)$, GKM+Yudovsky and Pilon (2009), and Sandoval and Kim (2014) approximations have error $\delta\left(\operatorname{In} r_{\infty}\right)$ of more than $50 \%$ at some sets of parameters (Fig. C.2), but yield a maximum of NRMSE $=5.1 \%$ for $\bar{K}_{\mathrm{d} \text {, dif }}(0-\rightarrow \tau)$. Such discrepancy may be explained by the combined impacts of $r_{\infty}, k$, $\bar{\mu}_{d}(\tau)$, and the form of equation chosen for $t(\tau)$ on the accuracy of the model $t(\tau)$ and $\bar{K}_{\mathrm{d} \text {, dif }}(0-\rightarrow \tau)$ equations. The models based on Hulst's model for $r_{\infty}(\# 6$ and 7$)$ yielded the best result with a total NRMSE of $0.06 \%$ and $2.1 \%$, respectively.

There is, however, an issue with the generalization of the analytical models. Recall that all the models tested before were calculated at a priori calculated values of $\bar{\mu}_{\mathrm{d} \text {,dif }}(\tau)$ [Eq. (B.1)]. To generalize these models, i.e., to make the models independent on numerical calculations, we have recalculated $\bar{K}_{\mathrm{d} \text {, dif }}(0-\rightarrow \tau)$ for $\bar{\mu}_{\mathrm{d} \text {, dif }}(\tau)$, computed by the analytical model, Eqs. (B.2)-(B.5). To strengthen a test of the ten selected models, we added a new $p(\theta) \# 4$ from Table 2 (at the same values of $\tau$ and $\omega_{0}$ that were used before), in addition to $p(\theta) \# 1-3$ in the second (test) step.

Table 4. Normalized (to the average) root-mean-square errors (\%) of analytically computed $\quad \bar{K}_{\text {d, dif }}(0-\rightarrow \tau)$ relatively corresponding values of $\bar{K}_{\text {d, dif }}(0-\rightarrow \tau)$ 
computed by the IPOL method for 64 optical depths at five values of $\omega_{0}$ (columns 3-7), four different $p(\theta)$ (left to right, top to bottom, in order, corresponds to the $p(\theta) \# 1-4$ in Table 2), and $\bar{\mu}_{\mathrm{d} \text {,dif }}(\tau)$ computed analytically (Eqs. B.2-B.5). Numeration of the models remains the same as in Table 3.

\begin{tabular}{|c|c|c|c|c|c|c|}
\hline$\# / \omega_{0}$ & $\begin{array}{c}\text { Model } \\
\text { equations }\end{array}$ & 0.0001 & 0.1 & 0.5 & 0.95 & 0.9999 \\
\hline 2 & $\begin{array}{l}\text { 16, A.1-A.3, } \\
\text { A.5, B.2-B.5, } \\
\text { C.3 }\end{array}$ & $\begin{array}{l}8.0,7.2 \\
7.1,7.1\end{array}$ & $\begin{array}{l}5.4,8.0 \\
8.5,9.1\end{array}$ & $\begin{array}{l}6.0,6.1 \\
3.7,4.4\end{array}$ & $\begin{array}{c}3.1,3.8 \\
13,5.4\end{array}$ & $\begin{array}{c}6.7,8.1 \\
14,5.9\end{array}$ \\
\hline 3 & $\begin{array}{c}\text { 15, } 16, \text { A.4- } \\
\text { A.6, B.2-B.5, } \\
\text { C.3, C.5, } \\
\text { C. } 20, \text { C. } 21\end{array}$ & $\begin{array}{l}8.0,7.2 \\
7.1,7.1\end{array}$ & $\begin{array}{r}5.4,9.9 \\
4.8,7.3\end{array}$ & $\begin{array}{l}6.6,10 \\
5.1,3.8\end{array}$ & $\begin{array}{c}3.2,3.6 \\
13,5.0\end{array}$ & $\begin{array}{r}6.5,8.0 \\
14,5.7\end{array}$ \\
\hline 4 & $\begin{array}{c}\text { 16, A.1-A.3, } \\
\text { A.5, B.2-B.5, } \\
\text { C. } 4, \text { C. } 5\end{array}$ & $\begin{array}{l}7.1,7.1 \\
7.1,7.1\end{array}$ & $\begin{array}{r}4.7,7.8 \\
8.5,9.1\end{array}$ & $\begin{array}{l}3.7,5.7 \\
3.7,4.4\end{array}$ & $\begin{array}{c}2.6,3.7 \\
14,6.6\end{array}$ & $\begin{array}{c}6.7,8.1 \\
14,5.9\end{array}$ \\
\hline 5 & $\begin{array}{c}\text { 15, } 16, \text { A.4- } \\
\text { A.6, B.2-B.5, } \\
\text { C. } 4, \text { C.5, } \\
\text { C. } 20, \text { C. } 21\end{array}$ & $\begin{array}{l}7.1,7.1 \\
7.1,7.1\end{array}$ & $\begin{array}{r}4.7,9.7 \\
7.1,7.3\end{array}$ & $\begin{array}{l}4.2,9.5 \\
5.1,3.8\end{array}$ & $\begin{array}{c}2.7,3.6 \\
14,6.1\end{array}$ & $\begin{array}{r}6.5,8.0 \\
14,5.7\end{array}$ \\
\hline 6 & $\begin{array}{l}\text { 16, A.1-A.3, } \\
\text { A.5, B.2-B.5, } \\
\text { C. } 7\end{array}$ & $\begin{array}{l}7.1,7.1 \\
7.1,7.1\end{array}$ & $\begin{array}{r}4.7,7.8 \\
8.5,9.1\end{array}$ & $\begin{array}{c}4.0,5.7 \\
3.7,4.4\end{array}$ & $\begin{array}{c}3.1,4.1 \\
14,7.7\end{array}$ & $\begin{array}{r}6.8,8.2 \\
13,6.8\end{array}$ \\
\hline 7 & $\begin{array}{c}\text { 15, 16, 31, } \\
\text { A.4-A.6, B.2- } \\
\text { B.5, C.5, } \\
\text { C. } 20, \text { C. } 21\end{array}$ & $\begin{array}{l}7.1,7.1 \\
7.1,7.1\end{array}$ & $\begin{array}{r}4.7,7.8 \\
8.5,9.1\end{array}$ & $\begin{array}{l}3.7,5.7 \\
3.7,4.4\end{array}$ & $\begin{array}{c}2.6,3.7 \\
14,6.6\end{array}$ & $\begin{array}{c}6.7,8.1 \\
13,5.9\end{array}$ \\
\hline 9 & $\begin{array}{c}\text { 16, A.1-A.3, } \\
\text { A.5, B.2-B.5, } \\
\text { C. } 2, \text { C. } 10\end{array}$ & $\begin{array}{l}7.1,7.1 \\
7.1,7.1\end{array}$ & $\begin{array}{r}4.7,7.8 \\
8.5,9.1\end{array}$ & $\begin{array}{l}3.0,5.0 \\
3.7,4.3\end{array}$ & $\begin{array}{c}2.5,3.6 \\
13,5.1\end{array}$ & $\begin{array}{c}6.7,8.1 \\
13,5.9\end{array}$ \\
\hline 10 & $\begin{array}{c}\text { 16, A.1-A.3, } \\
\text { A.5, B.2-B.5, } \\
\text { C. } 2 \text {, C.5, } \\
\text { C. } 11, \text { C. } 12\end{array}$ & $\begin{array}{l}7.1,7.1 \\
7.1,7.1\end{array}$ & $\begin{array}{r}4.7,7.8 \\
8.5,9.1\end{array}$ & $\begin{array}{l}3.7,5.7 \\
3.8,4.4\end{array}$ & $\begin{array}{c}2.6,3.6 \\
14,6.3\end{array}$ & $\begin{array}{r}6.7,8.1 \\
13,5.9\end{array}$ \\
\hline 11 & $\begin{array}{l}\text { 15, 16, A.4- } \\
\text { A.6, B.2-B.5, }\end{array}$ & $\begin{array}{l}7.1,7.1 \\
7.1,7.1\end{array}$ & $\begin{array}{l}\text { 4.7, 9.7, } \\
7.1,7.4\end{array}$ & $\begin{array}{l}4.1,9.6 \\
5.1,3.8\end{array}$ & $\begin{array}{c}2.6,3.6 \\
14,5.8\end{array}$ & $\begin{array}{c}6.5,8.0 \\
14,5.7\end{array}$ \\
\hline
\end{tabular}


C.2, C.5,

C.11, C.12,

C. 20, C.21

16, A.1-A.3,

12

A.5, B.2-B.5, C.2, C.5,

7.1, 7.1,

4.7, 7.8,

3.6, 5.6

2.6, 3.6,

6.7, 8.1,

C.11, C.13

$8.5,9.1$

$3.7,4.4$

$14,6.2$

$13,5.9$

15, 16, A.4-

13

A.6, B.2-B.5, C.2, C.5,

C.11, C.13,

7.1, 7.1,

4.7, 9.7,

4.1, 9.4

2.6, 3.6,

6.5, 8.0, $7.1,7.3$

$5.1,3.8$

$14,5.7$

$14,5.7$

C.20, C.21

16, A.1-A.3,

17

A.5, B.2-B.5, C.2, C.5,

C.11, C.18,

8.9, 9.0,

5.3, 9.5,

$3.5,5.5$,

2.6, 3.6,

6.7, 8.1, 11,11

$4.7,5.3 \quad 14,6.1$

11,21

C.19

15,16, A.4-

18

A.6, B.2-B.5, C.2, C.5,

C.11, C.18-

8.9, 9.0,

$5.3,12$,

$3.9,9.3$,

2.5, 3.6,

6.5, 8.0,

$9.1,9.3$

$4.4,4.5$

$14,5.6$

11,21 C. 21

16, A.1-A.3,

19

A.5, B.2-B.5, C.2, C.5,

C.11, C.20,

$7.1,7.1$

4.7, 7.8,

$3.5,5.5$,

2.6, 3.6,

$6.6,7.9$, $8.5,9.1$

$3.7,4.4$

$14,6.1$

$14,5.8$ C. 21

15,16, A.4-

20

A. 6 , B. $2-B .5$ C.5, C.20,

7.1, 7.1,

4.7, 9.7,

$3.9,9.4$,

2.6, 3.6

6.4, 7.8, C. 21

$7.1,7.3$

$5.1,3.8$

$14,5.6$

$14,5.7$

$21 \quad$ 16, A.1-A.3, $\quad 7.1,7.1$ C.2, C.22

$7.1,7.1$

4.7, 7.8,

3.0, 5.0,

2.5, 4.0,

6.7, 8.1, $8.5,9.1$

$3.7,4.3$

12, 3.7

$14,5.8$

15, 16, A.4-

22

A.6, B.2-B.5, C.5, C.20-

7.1, 7.1,

4.7, 10,

3.2, 8.7

2.5, 4.4

6.5, 8.0, C.22

$7.1,7.3$

$5.1,3.8$

$12,4.1$

$14,5.7$

$33 \quad 31$, A.1-A.3, 7.1, 7.1,

$4.7,7.8$

4.0, 5.7,

$3.1,4.1$,

$6.8,8.2$,

A.5, B.2-B.5

$7.1,7.1$

$8.5,9.1$

$3.7,4.4$

$14,7.7$

$13,6.8$

$34 \quad 15,31$, A.4- 7.1, 7.1,

4.7, 7.8,

3.7, 5.7,

2.6, 3.7,

$6.7,8.1$, A.6, B.2-B.5,

$8.5,9.1$

$3.7,4.4$

$14,6.6$

$13,5.9$ 
24, C.5, C. 20, C. 21

Results show some deterioration in comparison with results shown in Table 3 . This deterioration is caused by use an analytical algorithm for $\bar{\mu}_{\mathrm{d} \text {,dif }}(\tau)$ instead of preliminary computed values of $\bar{\mu}_{\mathrm{d} \text {, dif }}(\tau)$. Therefore, an upper limit of NRMSE increased from the 6.8\% found above for the Kokhanovsky and Hopkinson (2008) model (\#34 in Table 4) up to $21 \%$ (for the GKM+Yudovsky and Pilon (2009) model, \#17 and 18 in Table 5).

A positive aspect here is that the errors for a new phase function, \#4, are generally close in value to those for the $p(\theta) \# 1-3$ used for the algorithm development, which confirms a robustness of the spherical transmittance models. The $\bar{K}_{\mathrm{d} \text {, dif }}(0-\rightarrow \tau)$ models based on Hulst's model for $r_{\infty}(\# 6$ and 7$)$ yielded the best results, with a total NRMSE of $7.8 \%$ each.

Fig. 9 gives an example of how the best analytical models for $\bar{K}_{\mathrm{d} \text {, dif }}(0-\rightarrow \tau) / c$ agreed with the numerical calculations at different sets of $p(\theta), \tau$, and $\omega_{0}$. Figure confirms closeness between the Hulst-based models (\#6 and 7) and IPOL computations, with especial prominence for the very small (close to 0 ) and very large (more than 1000) values of $\tau$. 

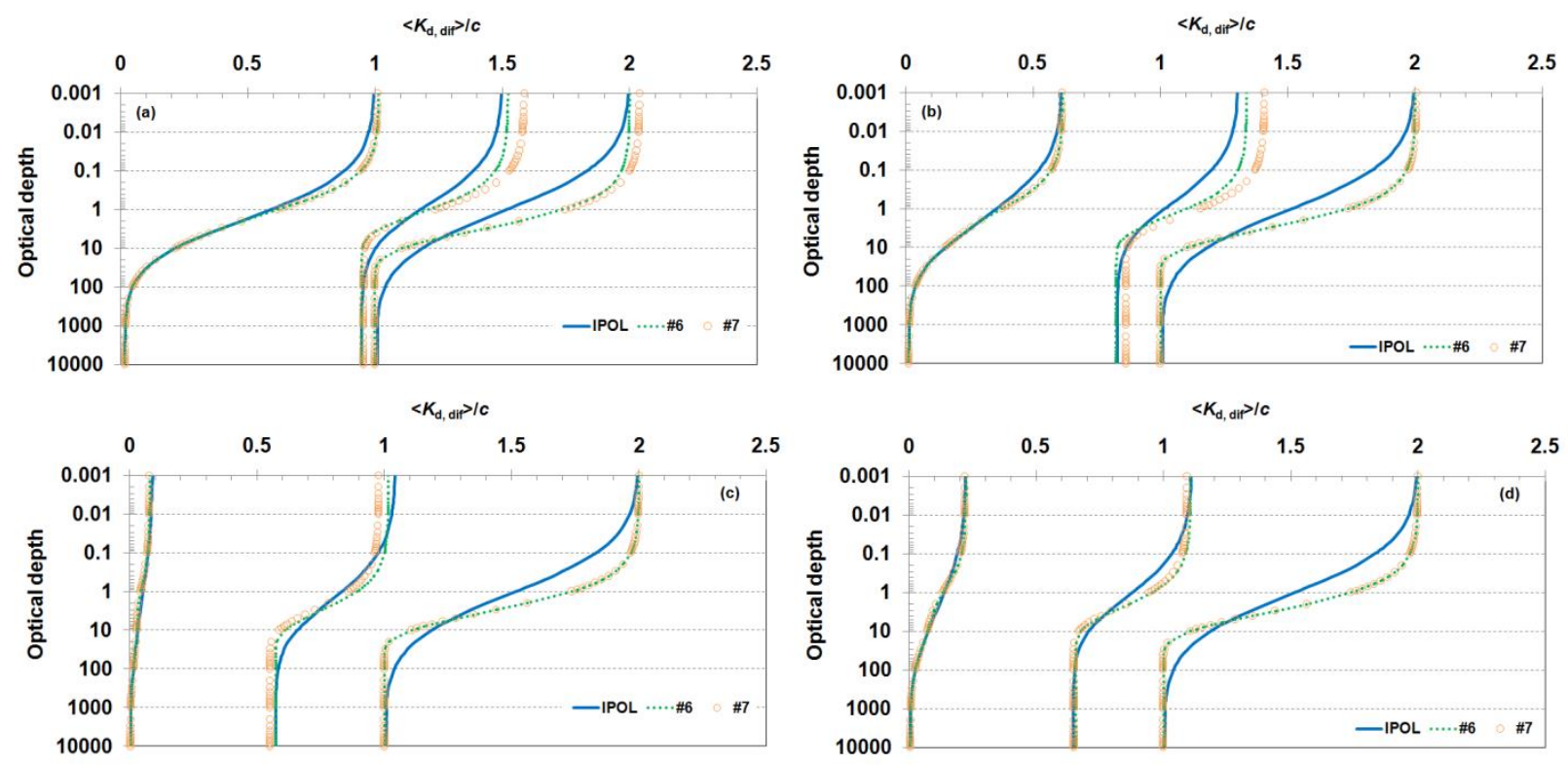

Fig. 9. The vertical profiles of $\bar{K}_{\mathrm{d} \text {, dif }}(0-\rightarrow \tau) / c$ computed by the IPOL (solid curves) and analytical models \#6 (dotted curves) and 7 (symbols) from Table 4 shown as the function of optical depth, $p(\theta)$ (letters a-d correspond to $p(\theta) \# 1-4$ from Table 2), and three values of $\omega_{0}: 0.0001,0.5$, and 0.9999 (right to left).

\subsection{Results for $T_{\mathrm{p}}(\tau)$ and $\bar{K}_{\mathrm{d} \text {, dir }}(0-\rightarrow \tau)$}

Results for $\bar{K}_{\mathrm{d} \text {,dir }}(0-\rightarrow \tau)$ at four selected incident angles $\theta_{\mathrm{i}}$ and $\bar{\mu}_{\mathrm{d}, \mathrm{dir}}\left(\theta_{\mathrm{i}}, \tau\right)$ calculated by Eq. (B.1) are presented in Table 5 . Selected parameters $\tau$, $\omega_{0}$, and $p(\theta)$ were the same as used for the Table 3, but the numeration of the models is new.

Table 5. Normalized (to the average) root-mean-square errors (\%) of analytically computed $\bar{K}_{\mathrm{d} \text {, dir }}(0-\rightarrow \tau)$ relatively corresponding values of $\bar{K}_{\mathrm{d} \text {, dir }}(0-\rightarrow \tau)$ computed by the IPOL method for 64 optical depths at four selected values of $\theta_{0}$, five values of $\omega_{0}$ 
(columns 3-7), three different $p(\theta)$ (left to right, top to bottom, in order, corresponds to the $p(\theta) \# 1-3$ in Table 2$)$, and $\bar{\mu}_{\mathrm{d} \text {,dir }}\left(\theta_{\mathrm{i}}, \tau\right)$ derived from the IPOL-based values of $R_{\infty}$ and $T(\tau)$ ], Eq. (B.1).

\begin{tabular}{|c|c|c|c|c|c|c|}
\hline$\# / \omega_{0}$ & $\begin{array}{c}\text { Model } \\
\text { equations }\end{array}$ & 0.0001 & 0.1 & 0.5 & 0.95 & 0.9999 \\
\hline \multicolumn{7}{|c|}{$\theta_{i}=0^{\circ}$} \\
\hline 1 & $\begin{array}{l}\text { 16, A.1-A.3, } \\
\text { A.5, B.2-B.5, } \\
\text { D.1 }\end{array}$ & $\begin{array}{c}0.31,0.31 \\
0.31\end{array}$ & $\begin{array}{c}0.37,0.35 \\
0.31\end{array}$ & $\begin{array}{c}0.052, \\
0.58,0.35\end{array}$ & $\begin{array}{c}3.7,0.20 \\
0.98\end{array}$ & $\begin{array}{c}2.3,1.7 \\
0.79\end{array}$ \\
\hline 2 & $\begin{array}{c}\text { 15, 16, A.4- } \\
\text { A.6, B.1, C.5, } \\
\text { C.20, C. } 21 \text {, } \\
\text { D. } 1\end{array}$ & $\begin{array}{c}0.31,0.30 \\
0.30\end{array}$ & $\begin{array}{c}0.38,2.6 \\
1.8\end{array}$ & $\begin{array}{c}0.91,5.0 \\
3.5\end{array}$ & $\begin{array}{c}3.6,0.95 \\
\quad 0.89\end{array}$ & $\begin{array}{c}2.5,1.8 \\
1.2\end{array}$ \\
\hline 3 & $\begin{array}{c}\text { 16, A.1-A.3, } \\
\text { B.1, A.5, C.9, } \\
\text { D.2 }\end{array}$ & $\begin{array}{l}4.5 \times 10^{-5} \\
4.9 \times 10^{-5} \\
4.2 \times 10^{-5}\end{array}$ & $\begin{array}{l}6.8 \times 10^{-4} \\
7.9 \times 10^{-4} \\
2.2 \times 10^{-5}\end{array}$ & $\begin{array}{c}0.22 \\
0.0063 \\
3.4 \times 10^{-5}\end{array}$ & $\begin{array}{c}4.9,2.2 \\
0.13\end{array}$ & $\begin{array}{c}119,107 \\
74\end{array}$ \\
\hline 4 & $\begin{array}{l}\text { 16, A.1-A.3, } \\
\text { A.5, B.1, D.3, } \\
\text { D. } 4\end{array}$ & $\begin{array}{l}4.4 \times 10^{-5} \\
4.8 \times 10^{-5} \\
4.1 \times 10^{-5}\end{array}$ & $\begin{array}{l}0.0057 \\
3.5 \times 10^{-4} \\
2.1 \times 10^{-5}\end{array}$ & $\begin{array}{c}0.0076 \\
0.020 \\
3.0 \times 10^{-4}\end{array}$ & $\begin{array}{c}1.8,0.092 \\
0.10\end{array}$ & $\begin{array}{c}8.0,2.1 \\
2.1\end{array}$ \\
\hline 5 & $\begin{array}{c}\text { 15, 16, A.4- } \\
\text { A.6, B.1, C.5, } \\
\text { C.20, C. } 21 \text {, } \\
\text { D.3, D.4 }\end{array}$ & $\begin{array}{c}1.1 \times 10^{-4} \\
0.0057 \\
0.017\end{array}$ & $\begin{array}{c}0.020,2.3 \\
2.0\end{array}$ & $\begin{array}{c}0.92,4.7 \\
3.7\end{array}$ & $\begin{array}{c}1.7,0.87 \\
0.11\end{array}$ & $\begin{array}{c}8.2,2.2 \\
2.5\end{array}$ \\
\hline 6 & $\begin{array}{c}\text { 16, A.1-A.3, } \\
\text { A.5, B.1, C.7, } \\
\text { D.5, D. } 6\end{array}$ & $\begin{array}{c}2.4 \times 10^{-4} \\
5.4 \times 10^{-4} \\
0.084\end{array}$ & $\begin{array}{l}0.032 \\
0.0019 \\
2.9 \times 10^{-5}\end{array}$ & $\begin{array}{c}0.85 \\
0.081 \\
5.1 \times 10^{-4}\end{array}$ & $\begin{array}{c}0.86,0.75 \\
0.015\end{array}$ & $\begin{array}{c}9.0,8.4 \\
7.5\end{array}$ \\
\hline 7 & $\begin{array}{c}\text { 16, A.4, A.5, } \\
\text { B.1, C.7, D.5, } \\
\text { D. } 6\end{array}$ & $\begin{array}{c}1.6 \times 10^{-4} \\
0.0054 \\
0.074\end{array}$ & $\begin{array}{c}0.024,2.3 \\
2.0\end{array}$ & $\begin{array}{c}0.76,4.6 \\
3.7\end{array}$ & $\begin{array}{c}0.76,0.64 \\
0.13\end{array}$ & $\begin{array}{c}\text { 8.8, 8.3 } \\
7.1\end{array}$ \\
\hline 8 & 17, B. 1 & $\begin{array}{l}0.010 \\
0.025 \\
0.023\end{array}$ & $10,10,3.3$ & $48,40,13$ & $92,85,51$ & $\begin{array}{c}128,120 \\
110\end{array}$ \\
\hline 9 & 18, B. 1 & $\begin{array}{c}0.0050 \\
0.023 \\
0.023\end{array}$ & $5.1,8.3,3.2$ & $22,31,12$ & $27,37,43$ & $\begin{array}{c}121,92 \\
68\end{array}$ \\
\hline 10 & 19, B.1 & $\begin{array}{c}0.0050 \\
0.022 \\
0.023\end{array}$ & $5.2,7.3,3.0$ & $25,26,11$ & $31,35,33$ & $19,17,17$ \\
\hline
\end{tabular}




\begin{tabular}{|c|c|c|c|c|c|}
\hline 20, B.1 & $\begin{array}{l}0.010 \\
0.024 \\
0.023\end{array}$ & $10,10,3.0$ & $47,39,11$ & $88,78,30$ & $\begin{array}{c}123,109 \\
84\end{array}$ \\
\hline 21, B.1 & $4.0,4.0,4.0$ & $\begin{array}{c}1.2,4.6 \\
0.74\end{array}$ & $\begin{array}{c}19,28 \\
8.5\end{array}$ & $29,34,40$ & $\begin{array}{c}126,94 \\
67\end{array}$ \\
\hline 22, B.1 & $\begin{array}{c}4.5,0.015 \\
0.022\end{array}$ & $\begin{array}{c}838,0.63 \\
2.4\end{array}$ & $\begin{array}{c}1632,11 \\
5.4\end{array}$ & $\begin{array}{c}1357,13 \\
22\end{array}$ & $\begin{array}{c}83,111 \\
81\end{array}$ \\
\hline $\begin{array}{l}\text { 23, A.1-A.3, } \\
\text { B.1 }\end{array}$ & $\begin{array}{l}4.4 \times 10^{-5} \\
4.8 \times 10^{-5} \\
4.2 \times 10^{-5}\end{array}$ & $\begin{array}{c}0.036 \\
0.0026 \\
0.15\end{array}$ & $\begin{array}{c}1.4,0.70 \\
9.3\end{array}$ & $12,24,224$ & $\begin{array}{c}19,74 \\
1744\end{array}$ \\
\hline $\begin{array}{c}\text { 24-26, A.1- } \\
\text { A.3, B.1 }\end{array}$ & $\begin{array}{l}4.4 \times 10^{-5} \\
4.8 \times 10^{-5} \\
4.1 \times 10^{-5}\end{array}$ & $\begin{array}{c}0.026 \\
0.0017 \\
2.2 \times 10^{-5}\end{array}$ & $\begin{array}{c}1.2,0.16 \\
6.7 \times 10^{-4}\end{array}$ & $\begin{array}{c}27,14 \\
0.35\end{array}$ & $85,86,76$ \\
\hline 27-29, B.1 & $\begin{array}{c}0.0091 \\
7.3 \times 10^{-4} \\
1.4 \times 10^{-4}\end{array}$ & $\begin{array}{c}10,0.85 \\
0.13\end{array}$ & $\begin{array}{c}69,9.1 \\
1.2\end{array}$ & $\begin{array}{c}329,122 \\
839\end{array}$ & $\begin{array}{c}615,401 \\
2161\end{array}$ \\
\hline $\begin{array}{l}\text { 30, A.1-A.3, } \\
\text { B.1 }\end{array}$ & $\begin{array}{l}4.4 \times 10^{-5}, \\
4.8 \times 10^{-5}, \\
4.1 \times 10^{-5}\end{array}$ & $\begin{array}{c}0.043 \\
0.0054 \\
2.5 \times 10^{-5}\end{array}$ & $\begin{array}{c}1.6,0.25 \\
0.0012\end{array}$ & $\begin{array}{c}22,5.1 \\
0.18\end{array}$ & $54,17,4.9$ \\
\hline
\end{tabular}

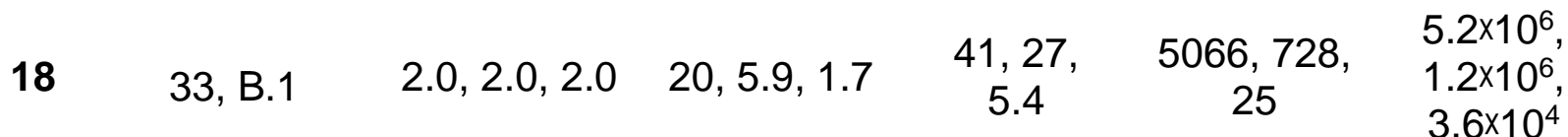

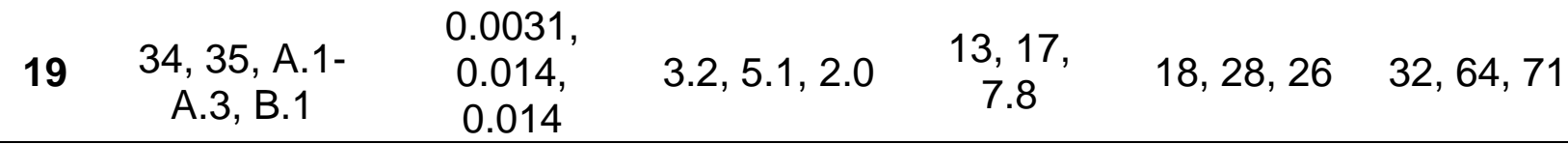
$\theta_{\mathrm{i}}=30^{\circ}$

16, A.1-A.3,

1 A.5, B.2-B.5,

D. 1

$0.53,0.53, \quad 0.67,0.61$

0.62

$0.55 \quad 0.98,0.61$

$2.1,0.38$

1.4, 0.91, 15, 16, A.4-
A.6, B.1, C.5,
2 C. 20, C.21,
$0.53,0.53, \quad 0.67,2.8$
1.4, 5.3,
D. 1
0.52
1.7
3.3
2.0, 0.58,
1.6, 1.0,

1.5

0.076

16, A.1-A.3, $4.8 \times 10^{-5}$

$3 \quad$ B.1, A.5, C.9, $3.8 \times 10^{-5}$, D. $2 \quad 4.2 \times 10^{-5}$

$0.0070, \quad 0.65$, $6.0 \times 10^{-4}$ 0.012 $2.9 \times 10^{-5}$ $1.1 \times 10^{-4}$ $5.8,0.64$ 0.24

119, 107, 16, A.1-A.3,

$4.6 \times 10^{-5}$

4 A.5, B.1, D.3,

$3.7 \times 10^{-5}$,

D. 4

$4.1 \times 10^{-5}$

0.0040 ,

$1.5 \times 10^{-4}$

0.020

$2.7 \times 10^{-5}$

15,16, A.4-

$1.0 \times 10^{-4}$

$5 \quad \mathrm{~A}, 6, \mathrm{~B} .1, \mathrm{C} .5$,

0.0058 , C.20, C.21, $\quad 0.017$

$0.018,2.4$,

2.0
0.017 ,

$6.9 \times 10^{-4}$

2.6, 0.078 , 0.076

9.1, 1.2, 0.42

$0.88,4.6, \quad 2.4,0.86, \quad 9.3,1.3$, 3.7 0.10

0.80 
D.3, D.4

$6 \begin{array}{cccccc}\text { 16, A.1-A.3, } & 1.4 \times 10^{-4}, & 0.036, & 1.0,0.16, & 3.2,1.3, & 1.4,1.5, \\ \text { A.S, B.1, C.7, } & 2.5 \times 10^{-4}, & 0.0032, & 6.2 \times 10^{-4} & 0.12 & 1.1\end{array}$ 16, A.4, A.5, $\quad 8.6 \times 10^{-5}, \quad 0.028,2.4, \quad 0.79,4.5, \quad 3.1,2.0, \quad 1.2,1.4$,

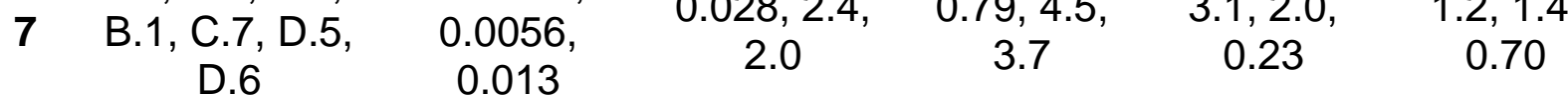

$8 \quad 17$, B. 1

0.010

$9 \quad 18$, B. $1 \quad 0.0050$,

0.025 , 0.023

$10,10,3.3 \quad 48,40,13$

$94,85,51$

130,122 , 108 0.023

$5.1,8.3,3.2 \quad 22,31,12 \quad 29,40,42$

109, 84, 0.023

$10 \quad 19$, B. $1 \quad 0.022$,

19, B.1

0.0050

$5.2,7.3,3.0$

$25,26,11$

$32,34,32$

$28,26,23$

0.023

1120 , B.1

0.010

0.024 ,

$10,10,3.0$

$47,39,11$

$90,79,30$

125,112 , 0.023

$12 \quad 21$, B. $1 \quad 4.0,4.0,4.0$

1.3, 4.6,

0.75

19,28

$29,38,40$

84

113, 85, $775,1.7, \quad 1545,6.3, \quad 1264,6.7, \quad 83,115$,

13 3.8, 0.017, 0.022 2.5 6.5 16 80

14

23, A.1-A.3
B.1

$4.6 \times 10^{-5}$,

$3.8 \times 10^{-5}$, $4.1 \times 10^{-5}$

0.041 0.0042 , 0.16

1.7, 0.65, 9.7

$16,23,226$

28,61 , $4.6 \times 10^{-5}$,

$15 \quad$ 24-26, A.1

$3.7 \times 10^{-5}$, $4.1 \times 10^{-5}$

0.028 , 0.0028 , $2.7 \times 10^{-5}$

1.4, 0.26,

$8.1 \times 10^{-4}$

29, 18, 0.54

$84,86,74$

$16 \quad 27-29$, B. $1 \quad 4.9,4.9,4.9$

$13,5.1,4.7$

$60,9.9$, 279,99 , 516, 300, 3.2 865 2004

17 30, A.1-A.3,
B.1 $4.6 \times 10^{-5}$,

$3.7 \times 10^{-5}$, $4.1 \times 10^{-5}$

0.040 0.0041 $2.9 \times 10^{-5}$

$1.5,0.17, \quad 18,2.4$, 0.0010 0.020 $40,7.0,12$

$18 \quad 33$, B. 1 $6.2,6.3,3.8$
0.0032, 1934,35, A.1 0.015 , A.3, B. 1 0.015 $\begin{array}{cc}35,21, & 4548,640, \\ 3.4 & 21\end{array}$ $4.5 \times 10^{6}$ $9.6 \times 10^{5}$, $3.5 \times 10^{4}$

\begin{tabular}{ccccccc} 
A.3, B.1 & $\begin{array}{c}0.015, \\
0.015\end{array}$ & $3.3,5.3,2.1$ & $\begin{array}{c}14,18, \\
8.2\end{array}$ & $22,33,27$ & $38,68,73$ \\
\hline \multicolumn{7}{c}{$\theta_{\mathrm{i}}=\mathbf{6 0}$} \\
\hline $\mathbf{1}$ & 16, A.1-A.3, & $2.6,2.6,2.6$ & $3.2,3.0,2.7$ & $5.1,4.3$, & $4.8,3.4$, & $2.0,1.5$,
\end{tabular}


A.5, B.2-B.5,

D. 1

3.0

5.0

1.0

15,16, A.4-

A.6, B.1, C.5,

2 C. 20, C. 21 , D. 1

16, A.1-A.3, $\quad 5.4 \times 10^{-5}$,

3 B.1, A.5, C.9, $5.4 \times 10^{-5}$,

D. 2

$5.0 \times 10^{-5}$

16, A.1-A.3,

4 A.5, B.1, D.3,

D. 4

$4.9 \times 10^{-5}$

$4.9 \times 10^{-5}$,

$4.5 \times 10^{-5}$

15,16, A.4-

5

A,6, B. 1, C.5,

C.20, C.21,

D.3, D.4

$1.1 \times 10^{-4}$,

0.0060 ,

0.017

$\begin{array}{cc}3.2,5.2,1.7 & 5.8,8.6 \\ 2.3\end{array}$

0.093

0.0035

$2.4 \times 10^{-5}$

5.9 0.084 ,

8112,93

180, 161, $0.018, \quad 0.57$

0.0037

$9.4 \times 10^{-5}$

0.10 ,

0.0064

4.9, 2.6,

4.9

1.8, 1.4,

0.60

16, A.1-A.3,

6 A.5, B.1, C.7,

$4.9 \times 10^{-5}$

$4.9 \times 10^{-5}$,

D.5, D. 6

$4.5 \times 10^{-5}$

16, A.4, A.5, $\quad 1.1 \times 10^{-4}$,

$7 \quad$ B.1, C.7, D.5, 0.0060,

D. 6

0.017

0.010

$8 \quad$ 17, B. 1

0.026 ,

0.024

$0.032,2.4$

$$
2.1
$$

1.4, 4.8

3.8

1.6

232

$2.5,0.26, \quad 0.63,0.24$

0.12

0.19

$9 \quad 18$, B. 1

0.0052 ,

0.024 ,

0.024

0.019 ,

0.0015 ,

$4.3 \times 10^{-5}$

1.9, 0.51, 0.0048

$11,7.9,1.3 \quad 14,14,13$

0.033, 2.4,

2.1

2.7, 5.1,

3.8

$11,7.1,1.2 \quad 14,14,12$

$10,10,3.4 \quad 50,41,13 \quad 102,95,52$

135, 132,

117

$5.3,8.6,3.3 \quad 24,32,12 \quad 39,57,44 \quad 84,85,87$

0.0052

1019, B. 1

0.023

$5.4,7.6,3$.

$26,27,11$

$42,43,33$

$54,54,48$

0.024

1120, B. 1

0.010

0.025

0.024

$10,10,3.1 \quad 49,40,11 \quad 99,90,31$

131,125

51

12

21, B. $1 \quad 4.2,4.2,4.2$

1.3, 4.7,

0.77

21,29

8.8

$37,55,42$

$84,84,85$

$538,6.5$,

1195,16 ,

11

96, 129,

0.024

3.2

$926,48,25$ 107

\section{4}

23, A.1-A.3

$4.9 \times 10^{-5}$,

$4.9 \times 10^{-5}$,

$4.6 \times 10^{-5}$

0.077
$0.021,0.18$

3.3,

$0.021,0.18 \quad 0.051,11$

$32,11,277$

54, 20,

$4.9 \times 10^{-5}$

0.037 ,

2.2, 1.0,

$36,32,2.7 \quad 78,86,80$ 


\section{$\begin{array}{llll}\text { A.3, B. } 1 \quad \begin{array}{lll}4.9 \times 10^{-5}, & 0.016, & 0.0070\end{array} & 4.5 \times 10^{-5} & 7.7 \times 10^{-5} & \end{array}$}

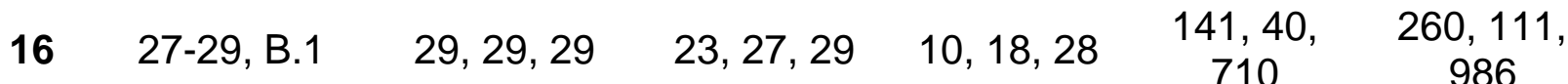

$17 \begin{array}{cccccc}40, \text { A.1-A.3, } & 4.9 \times 10^{-5}, & 0.018, & 0.56, & & 0.58,26 \text {, } \\ \text { B.1 } & 4.9 \times 10^{-5}, & 0.011, & 0.66, & 2.4,13,2.3 & 40\end{array}$

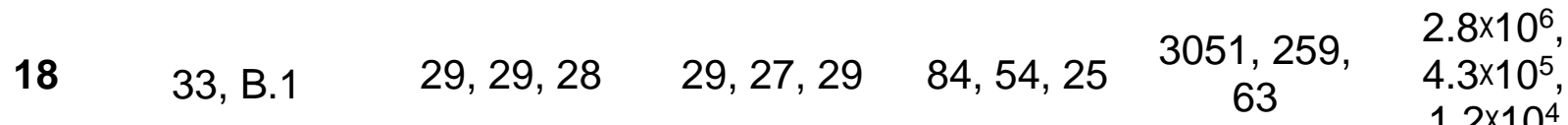

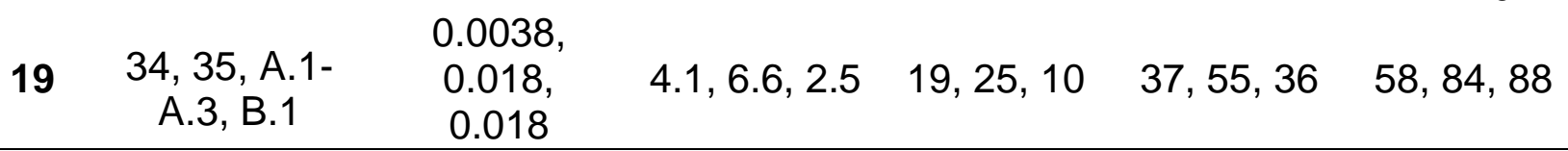

$\theta_{\mathrm{i}}=89.99^{\circ}$

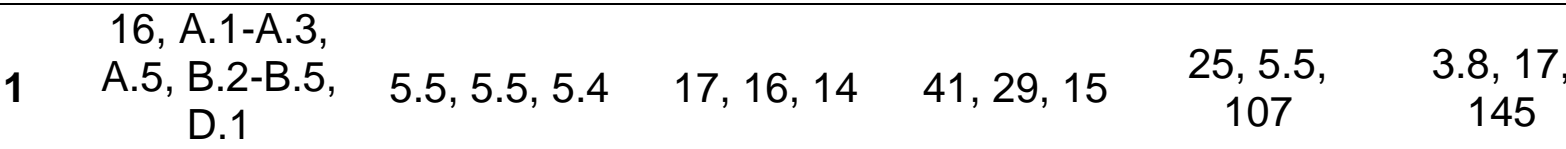
15,16, A.4-
A.6, B.1, C.5,
2 C. 20, C. 21 ,
$\begin{array}{ccccc}5.5,5.5,5.3 \quad 17,24,8.3 \quad 43,43, & 25,7.4, & 4.1,17, \\ & 4.0 & 107 & 146\end{array}$

16, A.1-A.3,

$3 \quad$ B.1, A.5, C.9, 22, 17, 15 D. 2

$632,675, \quad 606,608, \quad 421,440, \quad 318,320$, $\begin{array}{llll}2815 & 591 & 439 & 311\end{array}$

16, A.1-A.3, $\quad 1.3 \times 10^{-4}$

$4 \quad$ A.5, B.1, D.3, $\quad 7.0 \times 10^{-5}$ D. $4 \quad 1.6 \times 10^{-4}$

$\begin{array}{cccc}0.31,0.11, & 22,5.3, & 271,8.0, & 567,39 \\ 0.22 & 12 & 29 & 174\end{array}$ 15,16, A.4-
$4.4 \times 10^{-4}$,
0.016
$0.35,7.4$ 6.3
272, 9.9,
566, 39,

$5 \quad \begin{gathered}\text { A.6, B. } 1, \text { C. } 5 \\ \text { C. } 20, \text { C. } 21 \text {, } \\ \text { D.3, D. } 4\end{gathered}$

$6 \quad \begin{array}{ll}\text { 16, A.1-A.3, } & 1.3 \times 10^{-4}, \\ \text { A.5, B.1, C.7, } & 7.0 \times 10^{-5},\end{array}$

D.5, D. 6

$1.6 \times 10^{-4}$

16, A.4, A.5, $\quad 4.4 \times 10^{-4}$

$7 \quad$ B.1, C.7, D.5, 0.016,

D. 6

0.046

0.028 ,

8 17, B. 1

0.068 ,

0.063

$32,31,10$

0.014 ,

$9 \quad 18$, B. 1

0.064 ,

0.063

$16,26,9.7$

$80,106, \quad 162,221$,

218

186, 239,

279 


\begin{tabular}{|c|c|c|c|c|c|c|}
\hline 10 & 19, B.1 & $\begin{array}{l}0.014, \\
0.061 \\
0.062\end{array}$ & $16,23,9.3$ & $88,92,46$ & $\begin{array}{c}180,192 \\
203\end{array}$ & $\begin{array}{c}201,207 \\
250\end{array}$ \\
\hline 11 & 20, B.1 & $\begin{array}{l}\text { 0.027, } \\
0.067 \\
0.062\end{array}$ & $31,30,9.3$ & $\begin{array}{c}154,130 \\
46\end{array}$ & $\begin{array}{l}292,280 \\
201\end{array}$ & $\begin{array}{c}308,303 \\
244\end{array}$ \\
\hline 12 & 21, B.1 & $11,11,11$ & $4.1,14,2.0$ & $71,98,39$ & $\begin{array}{c}156,217 \\
215\end{array}$ & $\begin{array}{c}181,235 \\
278\end{array}$ \\
\hline 13 & 22, B.1 & $\begin{array}{l}6.2,0.081 \\
\quad 0.065\end{array}$ & $4.1,46,12$ & $\begin{array}{c}16,270 \\
70\end{array}$ & $\begin{array}{c}13,0.87 \\
0.19\end{array}$ & $\begin{array}{l}1.8,0.81 \\
0.045\end{array}$ \\
\hline 14 & $\begin{array}{l}\text { 23, A.1-A.3, } \\
\text { B.1 }\end{array}$ & $\begin{array}{l}1.3 \times 10^{-4}, \\
7.0 \times 10^{-5} \\
1.6 \times 10^{-4}\end{array}$ & $\begin{array}{c}0.28,0.27 \\
0.12\end{array}$ & $\begin{array}{c}19,15 \\
0.49\end{array}$ & $\begin{array}{c}162,102 \\
113\end{array}$ & $\begin{array}{c}201,120 \\
285\end{array}$ \\
\hline 15 & $\begin{array}{c}\text { 24-26, A.1- } \\
\text { A.3, B.1 }\end{array}$ & $\begin{array}{l}1.3 \times 10^{-4} \\
7.0 \times 10^{-5} \\
1.6 \times 10^{-4}\end{array}$ & $\begin{array}{c}0.33,0.20 \\
0.24\end{array}$ & $11,11,15$ & $53,39,153$ & $\begin{array}{c}62,7.7 \\
169\end{array}$ \\
\hline 16 & 27-29, B.1 & $\begin{array}{l}228,229 \\
234\end{array}$ & $\begin{array}{c}190,195 \\
221\end{array}$ & $\begin{array}{c}114,145 \\
195\end{array}$ & $\begin{array}{c}52,127 \\
507\end{array}$ & $\begin{array}{c}50,130 \\
285\end{array}$ \\
\hline 17 & $\begin{array}{l}\text { 30, A.1-A.3, } \\
\text { B.1 }\end{array}$ & $\begin{array}{l}1.3 \times 10^{-4} \\
7.0 \times 10^{-5} \\
1.6 \times 10^{-4}\end{array}$ & $\begin{array}{c}0.21,0.27 \\
0.24\end{array}$ & $13,16,15$ & $\begin{array}{c}103,140 \\
160\end{array}$ & $\begin{array}{c}134,173 \\
244\end{array}$ \\
\hline 18 & 33, B.1 & $\begin{array}{c}278,277, \\
274\end{array}$ & $\begin{array}{c}314,313 \\
\quad 302\end{array}$ & $\begin{array}{c}312,312 \\
296\end{array}$ & $\begin{array}{c}299,312 \\
300\end{array}$ & $\begin{array}{c}36910, \\
3536,298\end{array}$ \\
\hline 19 & $\begin{array}{c}\text { 34, 35, A.1- } \\
\text { A.3, B.1 }\end{array}$ & $\begin{array}{l}0.014, \\
0.063 \\
0.063\end{array}$ & $16,26,9.7$ & $\begin{array}{c}80,106 \\
49\end{array}$ & $\begin{array}{c}162,221 \\
218\end{array}$ & $\begin{array}{c}186,239 \\
279\end{array}$ \\
\hline
\end{tabular}

Table 5 demonstrates some similar features for the plane transmittance models as were found for the spherical transmittance. For example, almost all models (excluding models \#1 and 2) improve their performance with an increase the contribution of

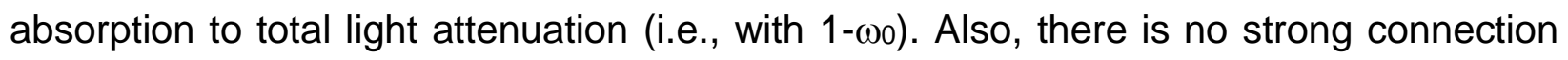
between performance of albedo and transmittance for all models: even though the HKS 2 model has shown excellent results for both plane albedo of infinite layer (Figs. D.1D.3, D.5-D.7) and plane transmittance at $\theta_{i} \leq 60^{\circ}$ (Table 5), the exponential and replacement models may give errors of $\delta\left(\ln R_{p \infty}\right)$ greater than $50 \%$ at some sets of 
parameters, but yield a maximum of NRMSE of only $14 \%$ for $\bar{K}_{\mathrm{d} \text {, dir }}(0-\rightarrow \tau)$ at the same range of incident angles.

However, only six models (\#1, 2, 4-7) among 19 have shown an acceptable accuracy at $\theta_{\mathrm{i}} \leq 60^{\circ}, \omega_{0} \leq 0.9999$, and three $p(\theta)$; one model (\#17) has shown an acceptable accuracy at $\theta_{\mathrm{i}} \leq 60^{\circ}$ and $\omega_{0} \leq 0.95$; and no models yielded acceptable results at extremely large $\theta_{i}$ close to $90^{\circ}$. Therefore, we exclude an angle $\theta_{i}=89.99^{\circ}$ from further consideration. The best $\bar{K}_{\mathrm{d} \text {, dir }}(0-\rightarrow \tau)$ model based on the HKS 2 approximation for $R_{p_{\infty}}(\# 4)$ yielded a total NRMSE of $0.95 \%, 1.1 \%, 0.48 \%$, and $37 \%$ for the $\theta$ i of $0^{\circ}, 30^{\circ}, 60^{\circ}$, and $89.99^{\circ}$, respectively. Another $\bar{K}_{\mathrm{d} \text {, dir }}(0-\rightarrow \tau)$ model also based on the HKS 2 approximation for $R_{p_{\infty}}(\# 5)$ has shown a total NRMSE of $2.3 \%, 2.3 \%$, $2.1 \%$, and $37 \%$ for the same angles.

Table 6 shows errors for the best $\bar{K}_{\mathrm{d} \text {, dir }}(0-\rightarrow \tau)$ models at $\theta_{\mathrm{i}} \leq 60^{\circ}$, but with the $\bar{\mu}_{\mathrm{d} \text {, dir }}\left(\theta_{\mathrm{i}}, \tau\right)$ computed by the analytical model, Eqs. (B.2)-(B.5). As before, we have also added a new $p(\theta) \# 4$ from Table 2, keeping the same values of $\tau$ and $\omega_{0}$, that were used for Table 5 .

Table 6. Normalized (to the average) root-mean-square errors (\%) of analytically computed $\bar{K}_{\mathrm{d} \text {, dir }}(0-\rightarrow \tau)$ relatively corresponding values of $\bar{K}_{\mathrm{d} \text {, dir }}(0-\rightarrow \tau)$ computed by the IPOL method for 64 optical depths at three values of $\theta_{i}$, five values of $\omega_{0}$ (columns 3-7), four different $p(\theta)$ (left to right, top to bottom, in order, corresponds to the 
$p(\theta) \# 1-4$ in Table 2$)$, and $\bar{\mu}_{\mathrm{d} \text {,dir }}(\tau)$ computed analytically (Eqs. 20-24). Numeration of the models kept the same as in Table 5.

\begin{tabular}{|c|c|c|c|c|c|c|}
\hline$\# / \omega_{0}$ & $\begin{array}{c}\text { Model } \\
\text { equations }\end{array}$ & 0.0001 & 0.1 & 0.5 & 0.95 & 0.9999 \\
\hline \multicolumn{7}{|c|}{$\theta_{i}=0^{\circ}$} \\
\hline 1 & $\begin{array}{l}\text { 16, A.1-A.3, } \\
\text { A.5, B.2-B.5, } \\
\text { D.1 }\end{array}$ & $\begin{array}{l}0.30,0.31 \\
0.32,0.32\end{array}$ & $\begin{array}{l}3.4,2.1 \\
3.8,2.7\end{array}$ & $\begin{array}{l}4.1,5.1 \\
2.3,4.7\end{array}$ & $\begin{array}{l}6.2,5.3 \\
9.3,3.4\end{array}$ & $\begin{array}{l}15,13 \\
35,12\end{array}$ \\
\hline 2 & $\begin{array}{c}\text { 15, 16, A.4- } \\
\text { A.6, B.2-B.5, } \\
\text { C.5, C. } 20, \\
\text { C. } 21, \text { D. } 1\end{array}$ & $\begin{array}{l}0.30,0.31 \\
0.30,0.30\end{array}$ & $\begin{array}{l}3.4,1.9 \\
5.4,4.3\end{array}$ & $\begin{array}{l}3.7,8.4 \\
2.2,3.7\end{array}$ & $\begin{array}{l}6.1,5.5 \\
9.3,3.3\end{array}$ & $\begin{array}{l}15,13 \\
35,13\end{array}$ \\
\hline 4 & $\begin{array}{c}\text { 16, A.1-A.3, } \\
\text { A.5, B.2-B.5, } \\
\text { D.3, D. } 4\end{array}$ & $\begin{array}{l}0.0050 \\
0.011 \\
0.015 \\
0.014\end{array}$ & $\begin{array}{c}3.7,2.3 \\
4.1,3.0\end{array}$ & $\begin{array}{l}4.0,4.6 \\
2.0,4.3\end{array}$ & $\begin{array}{c}4.4,5.3 \\
9.8,3.8\end{array}$ & $\begin{array}{l}20,13 \\
35,13\end{array}$ \\
\hline 5 & $\begin{array}{c}\text { 15, 16, A.4- } \\
\text { A.6, B.2-B.5, } \\
\text { C.5, C.20, } \\
\text { C. } 21, \text { D.3, } \\
\text { D.4 }\end{array}$ & $\begin{array}{l}0.0051 \\
0.0061 \\
0.0053 \\
0.0044\end{array}$ & $\begin{array}{l}3.7,1.9 \\
5.6,4.6\end{array}$ & $\begin{array}{l}3.7,7.8 \\
2.3,3.3\end{array}$ & $\begin{array}{l}4.3,5.5 \\
9.8,3.9\end{array}$ & $\begin{array}{l}20,13 \\
35,13\end{array}$ \\
\hline 6 & $\begin{array}{l}\text { 16, A.1-A.3, } \\
\text { A.5, B.2-B.5, } \\
\text { C.7, D.5, D.6 }\end{array}$ & $\begin{array}{l}0.0049 \\
0.0010 \\
0.088 \\
0.012\end{array}$ & $\begin{array}{l}3.7,2.3 \\
4.1,3.0\end{array}$ & $\begin{array}{l}4.8,4.5 \\
2.0,4.3\end{array}$ & $\begin{array}{c}3.5,5.4 \\
10,3.7\end{array}$ & $\begin{array}{c}4.6,8.6 \\
37,8.0\end{array}$ \\
\hline 7 & $\begin{array}{c}\text { 16, A.4, A.5, } \\
\text { B.2-B.5, C.7, } \\
\text { D.5, D.6 }\end{array}$ & $\begin{array}{c}0.0050 \\
0.0056 \\
0.076 \\
0.0045\end{array}$ & $\begin{array}{l}3.7,1.9 \\
5.6,4.6\end{array}$ & $\begin{array}{l}4.4,7.7 \\
2.3,3.3\end{array}$ & $\begin{array}{c}3.4,5.4 \\
10,3.7\end{array}$ & $\begin{array}{c}4.8,8.6 \\
37,8.0\end{array}$ \\
\hline 17 & $\begin{array}{c}\text { 30, A.1-A.3, } \\
\text { B.2-B.5 }\end{array}$ & $\begin{array}{c}0.0050 \\
0.011 \\
0.015 \\
0.014 \\
\end{array}$ & $\begin{array}{l}3.6,2.3 \\
4.1,3.0\end{array}$ & $\begin{array}{l}3.0,4.8 \\
2.0,4.3\end{array}$ & $\begin{array}{l}18,7.4 \\
9.7,3.4\end{array}$ & $\begin{array}{l}36,12 \\
35,7.9\end{array}$ \\
\hline \multicolumn{7}{|c|}{$\theta_{\mathrm{i}}=\mathbf{3 0 ^ { \circ }}$} \\
\hline 1 & $\begin{array}{l}\text { 16, A.1-A.3, } \\
\text { A.5, B.2-B.5, } \\
\text { D.1 }\end{array}$ & $\begin{array}{l}5.0,4.9 \\
6.0,4.9\end{array}$ & $\begin{array}{l}5.2,3.6 \\
5.2,3.8\end{array}$ & $\begin{array}{l}4.5,4.8 \\
2.7,4.6\end{array}$ & $\begin{array}{l}1.5,5.9 \\
4.7,2.9\end{array}$ & $\begin{array}{l}5.0,16 \\
21,9.5\end{array}$ \\
\hline 2 & $\begin{array}{r}\text { 15, 16, A.4- } \\
\text { A.6, B.2-B.5, }\end{array}$ & $\begin{array}{l}5.0,4.9 \\
6.0,4.9\end{array}$ & $\begin{array}{l}5.2,2.3 \\
6.9,5.7\end{array}$ & $\begin{array}{l}3.9,7.6 \\
3.7,3.8\end{array}$ & $\begin{array}{l}1.4,6.3 \\
4.7,3.2\end{array}$ & $\begin{array}{c}5.2,16 \\
21,10\end{array}$ \\
\hline
\end{tabular}


C.5, C.20, C. 21 , D. 1

$\begin{array}{cccccc}\text { 16, A.1-A.3, } & 5.0,4.8, & 5.6,3.9, & 4.9,4.0, & 1.9,6.2, & 13,16, \\ \text { A.5, B.2-B.5, } & 6.0,4.9 & 5.6,4.1 & 2.1,3.8 & 5.9,4.2 & 21,9.4 \\ \text { D.3, D.4 } & & & & & \end{array}$
15, 16, A.4-

\begin{tabular}{|c|c|c|c|c|c|c|}
\hline 5 & $\begin{array}{c}\text { A.6, B.2-B.5, } \\
\text { C.5, C. } 20 \text {, } \\
\text { C. } 21, \text { D. } 3 \text {, } \\
\text { D. } 4\end{array}$ & $\begin{array}{l}5.0,4.9 \\
6.0,4.9\end{array}$ & $\begin{array}{l}5.5,2.4 \\
7.3,6.1\end{array}$ & $\begin{array}{l}4.3,6.6 \\
3.8,3.1\end{array}$ & $\begin{array}{l}1.8,6.6 \\
6.0,4.6\end{array}$ & $\begin{array}{l}13,16 \\
20,10\end{array}$ \\
\hline 6 & $\begin{array}{l}\text { 16, A.1-A.3, } \\
\text { A.5, B.2-B.5, } \\
\text { C.7, D.5, D.6 }\end{array}$ & $\begin{array}{l}5.0,5.0 \\
5.0,5.0\end{array}$ & $\begin{array}{l}5.6,3.9 \\
5.6,4.1\end{array}$ & $\begin{array}{l}5.6,3.9 \\
2.1,3.8\end{array}$ & $\begin{array}{l}2.5,7.0 \\
6.1,4.6\end{array}$ & $\begin{array}{l}2.3,14, \\
22,8.0\end{array}$ \\
\hline 7 & $\begin{array}{c}\text { 16, A.4, A.5, } \\
\text { B.2-B.5, C.7, } \\
\text { D.5, D.6 }\end{array}$ & $\begin{array}{l}5.0,5.0 \\
5.0,5.0\end{array}$ & $\begin{array}{l}5.6,2.4 \\
7.3,6.1\end{array}$ & $\begin{array}{l}5.1,6.5 \\
3.8,3.1\end{array}$ & $\begin{array}{l}2.3,7.5 \\
6.1,5.0\end{array}$ & $\begin{array}{l}2.5,14, \\
22,8.1\end{array}$ \\
\hline 17 & $\begin{array}{c}\text { 30, A.1-A.3, } \\
\text { B.2-B.5 }\end{array}$ & $\begin{array}{l}5.0,4.8 \\
6.0,4.9 \\
\end{array}$ & $\begin{array}{l}5.5,3.9 \\
5.6,4.1\end{array}$ & $\begin{array}{l}4.0,4.1 \\
2.1,3.9 \\
\end{array}$ & $\begin{array}{l}19,4.9 \\
6.0,3.7 \\
\end{array}$ & $\begin{array}{l}35,9.9, \\
7.6,8.5 \\
\end{array}$ \\
\hline \multicolumn{7}{|c|}{$\theta \mathrm{i}=60^{\circ}$} \\
\hline 1 & $\begin{array}{c}\text { 16, A.1-A.3, } \\
\text { A.5, B.2-B.5, } \\
\text { D. } 1\end{array}$ & $\begin{array}{l}11,11 \\
12,12\end{array}$ & $\begin{array}{l}6.7,4.5 \\
8.6,6.1\end{array}$ & $\begin{array}{l}4.5,3.7 \\
6.3,5.4\end{array}$ & $\begin{array}{l}25,15 \\
20,19\end{array}$ & $\begin{array}{l}37,28 \\
33,37\end{array}$ \\
\hline 2 & $\begin{array}{c}\text { 15, 16, A.4- } \\
\text { A.6, B.2-B.5, } \\
\text { C.5, C. } 20 \text {, } \\
\text { C. } 21, \text { D. } 1\end{array}$ & $\begin{array}{l}11,11 \\
12,12\end{array}$ & $\begin{array}{c}6.7,3.5 \\
10,7.9\end{array}$ & $\begin{array}{l}4.5,6.8 \\
7.2,5.0\end{array}$ & $\begin{array}{l}25,15 \\
21,20\end{array}$ & $\begin{array}{l}28,33 \\
35,37\end{array}$ \\
\hline 4 & $\begin{array}{c}\text { 16, A.1-A.3, } \\
\text { A.5, B.2-B.5, } \\
\text { D.3, D.4 }\end{array}$ & $\begin{array}{l}11,11 \\
12,11\end{array}$ & $\begin{array}{l}7.9,5.8 \\
9.8,7.3\end{array}$ & $\begin{array}{l}4.9,3.1 \\
5.4,3.4\end{array}$ & $\begin{array}{l}22,17 \\
24,23\end{array}$ & $\begin{array}{l}35,29 \\
33,38\end{array}$ \\
\hline 5 & $\begin{array}{c}15,16, \text { A.4- } \\
\text { A.6, B.2-B.5, } \\
\text { C.5, C.20, } \\
\text { C. } 21, \text { D. } 3 \\
\text { D. } 4\end{array}$ & $\begin{array}{l}11,11 \\
12,11\end{array}$ & $\begin{array}{c}7.9,3.9 \\
11,9.3\end{array}$ & $\begin{array}{l}4.3,3.2 \\
7.8,3.7\end{array}$ & $\begin{array}{l}22,17 \\
24,23\end{array}$ & $\begin{array}{l}35,29 \\
33,38\end{array}$ \\
\hline 6 & $\begin{array}{l}\text { 16, A.1-A.3, } \\
\text { A.5, B.2-B.5, } \\
\text { C.7, D.5, D.6 }\end{array}$ & $\begin{array}{l}11,11 \\
11,11\end{array}$ & $\begin{array}{l}7.9,5.8 \\
9.8,7.3\end{array}$ & $\begin{array}{l}4.4,2.9 \\
5.4,3.4\end{array}$ & $\begin{array}{l}32,11 \\
23,19\end{array}$ & $\begin{array}{l}52,18 \\
25,28\end{array}$ \\
\hline 7 & $\begin{array}{c}\text { 16, A.4, A.5, } \\
\text { B.2-B.5, C.7, } \\
\text { D.5, D. } 6\end{array}$ & $\begin{array}{l}11,11 \\
11,11\end{array}$ & $\begin{array}{c}7.9,3.9 \\
11,9.3\end{array}$ & $\begin{array}{l}3.9,3.5 \\
7.8,3.7\end{array}$ & $\begin{array}{l}32,12 \\
23,20\end{array}$ & $\begin{array}{l}52,18 \\
25,29\end{array}$ \\
\hline 17 & $\begin{array}{c}\text { 30, A.1-A.3, } \\
\text { B.2-B.5 }\end{array}$ & $\begin{array}{l}11,11 \\
12,11\end{array}$ & $\begin{array}{l}7.9,5.8 \\
9.8,7.3\end{array}$ & $\begin{array}{l}4.9,3.6 \\
5.4,3.4\end{array}$ & $\begin{array}{l}22,29 \\
26,28\end{array}$ & $\begin{array}{l}35,49 \\
61,58\end{array}$ \\
\hline
\end{tabular}


Results show some deterioration in comparison with results shown in Table 5, especially at $\theta_{\mathrm{i}}=60^{\circ}$ and $\omega_{0}=0.9999$. This deterioration may be explained by the use of the analytical algorithm for $\bar{\mu}_{\mathrm{d}, \mathrm{dir}}(\tau)$ instead of the values based on the numerical (IPOL) calculations. A connection between large errors found at high values of $\theta_{\mathrm{i}}$ in $\bar{\mu}_{\mathrm{d} \text {,dir }}(\tau)$ (Fig. B.2) and large errors in $\bar{K}_{\mathrm{d} \text {, dir }}(0-\rightarrow \tau)$ at the same angular conditions appears to be obvious.

The upper value of NRMSE increased from the $54 \%$ found above for the Pottier et al. (2005) model (\#17 in Table 5) at numerically determined $\bar{\mu}_{\text {d,dir }}(\tau)$ to $61 \%$ for the same method at analytically determined $\bar{\mu}_{\mathrm{d} \text {,dir }}(\tau)$. A positive finding here is that the errors for a new phase function, \#4, generally have the same order of amplitude as for $p(\theta) \# 1-3$ used for the algorithm development. This finding clearly confirms a robustness of the plane transmittance models used in the study.

The best $\bar{K}_{\text {d, dir }}(0-\rightarrow \tau)$ model is based on the HKS 2 model for $R_{p \infty}(\# 4)$ and yields a total NRMSE of $3.9 \%, 5.9 \%$, and $12 \%$ for the $\theta_{i}$ of $0^{\circ}, 30^{\circ}$, and $60^{\circ}$, respectively. Another $\bar{K}_{\mathrm{d} \text { dir }}(0-\rightarrow \tau)$ model also based on the HKS 2 model for $R_{\mathrm{p} \infty}(\# 5)$ has shown a total NRMSE of $4.5 \%, 6.3 \%$, and $12 \%$ for the same angles.

Figures 10-12 show performance of the best analytical models for $\bar{K}_{\mathrm{d} \text {, dir }}(0-\rightarrow \tau) / c$ at different sets of $\theta \mathrm{i}, p(\theta), \tau$, and $\omega 0$. Here, models \#4 and 5 with the independently computed $\bar{\mu}_{\mathrm{d} \text {,dir }}(\tau)$ were used to show vertical profiles of $\bar{K}_{\mathrm{d} \text {, dir }}(0-\rightarrow \tau) / c$ together with the similar relationships computed by the IPOL algorithm. Corresponding relative errors 
of models \#4 and 5 are shown in Figs. 13-15. Analysis of errors shows that a majority of errors lie in the range of $\pm 20 \%$, but errors may be larger at high values of $\theta_{i}$ and $\omega_{0}$ close to 1 .

An additional test for $T_{\mathrm{p}}(\tau)$ and $\bar{K}_{\mathrm{d} \text {, dir }}(0-\rightarrow \tau) / c$ has been performed for the HenyeyGreenstein phase function (Henyey and Greenstein, 1941) for the case of a normal light incidence and at selected $\tau, g$ and $\omega_{0}$ corresponding to the values published by Prahl (1995). All computations by Prahl (1995) were performed by the adding-doubling method and published with four digits. The values of $T_{\mathrm{p}}(\tau)$ computed by IPOL, Prahl (1995), and HKS 2 approximation (\#4 in Table 6) are compared in Table 7. Even though the four digits of $T_{\mathrm{p}}(\tau)$ presented by Prahl (1995) are almost completely identical to the values found by IPOL algorithm, this accuracy is not high enough at small $\tau$ when a conversion to $\bar{K}_{\mathrm{d} \text {, dir }}(0-\rightarrow \tau) / c$ is performed.
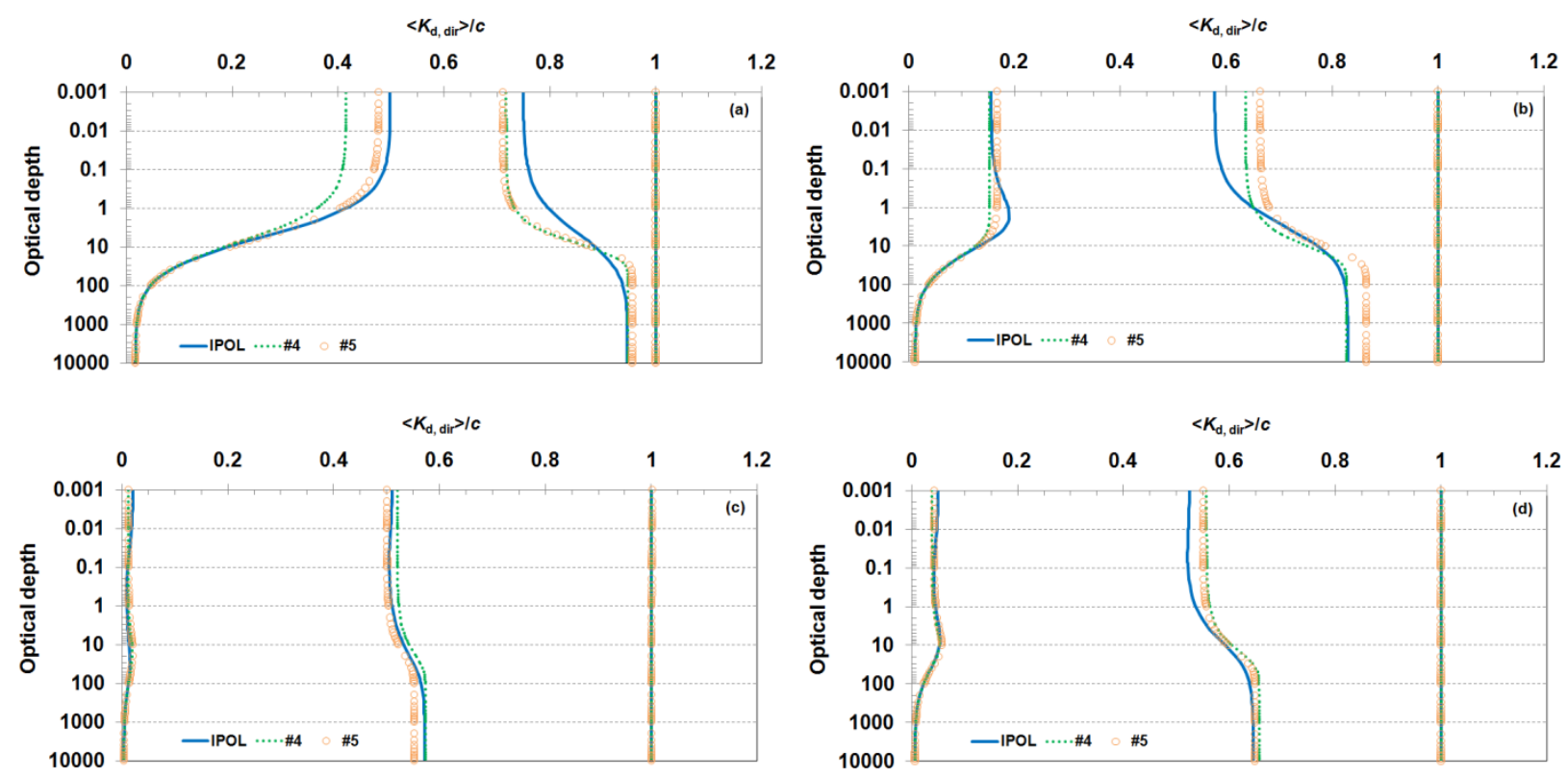
Fig. 10. The vertical profiles of $\bar{K}_{\mathrm{d} \text {, dir }}(0-\rightarrow \tau) / c$ at $\theta_{\mathrm{i}}=0^{\circ}$ computed by the IPOL (solid blue curves), and analytical models \#4 (dotted green curves) and 5 (orange symbols) from Table 6 as a function of optical depth, $p(\theta)$ (letters a-d correspond to $p(\theta) \# 1-4$ from Table 2), and three values of $\omega_{0}: 0.0001,0.5$, and 0.9999 (right to left).
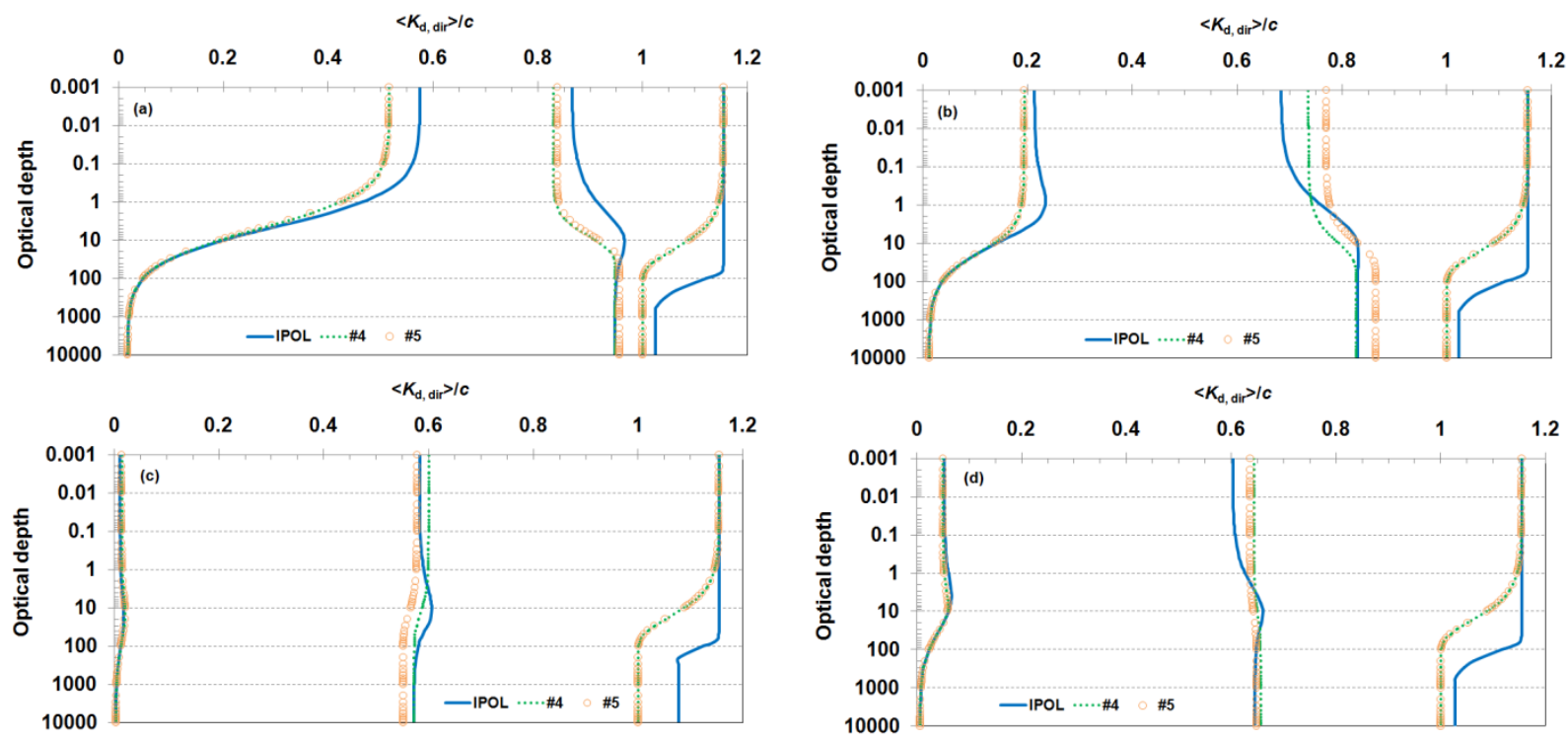

Fig. 11. The same as Fig. 10, but for $\theta_{i}=30^{\circ}$.
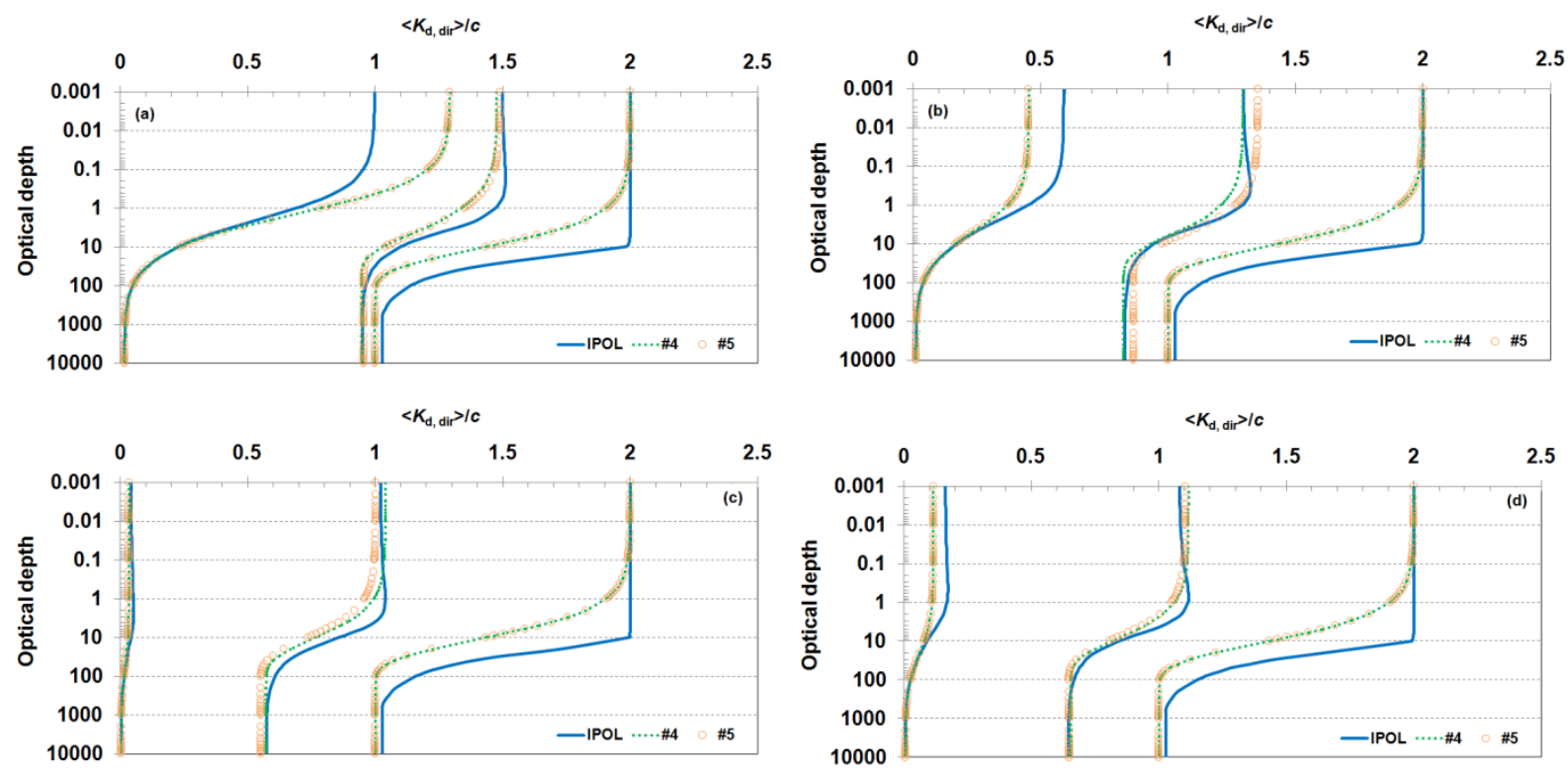
Fig. 12. The same as Fig. 10, but for $\theta_{\mathrm{i}}=60^{\circ}$.
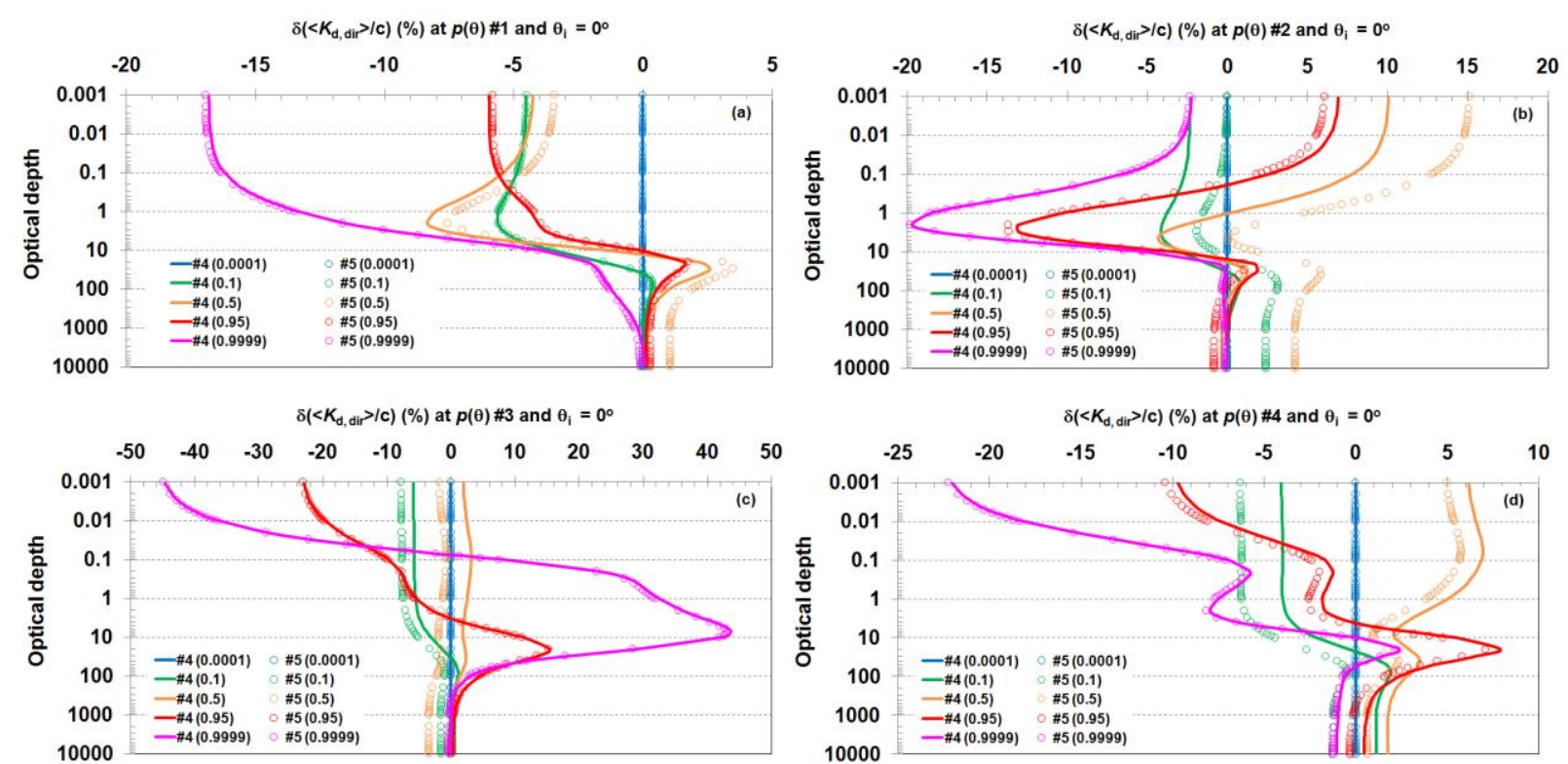

Fig. 13. The vertical profiles of relative errors in $\bar{K}_{\mathrm{d} \text {, dir }}(0-\rightarrow \tau) / c$ derived by the models

\#4 and 5 (Table 7) relative to IPOL-based values at $\theta_{i}=0^{\circ}$, four $p(\theta)$ (letters a-d correspond to $p(\theta) \# 1-4$ from Table 2), and five values of $\omega_{0}$ shown in the legends.
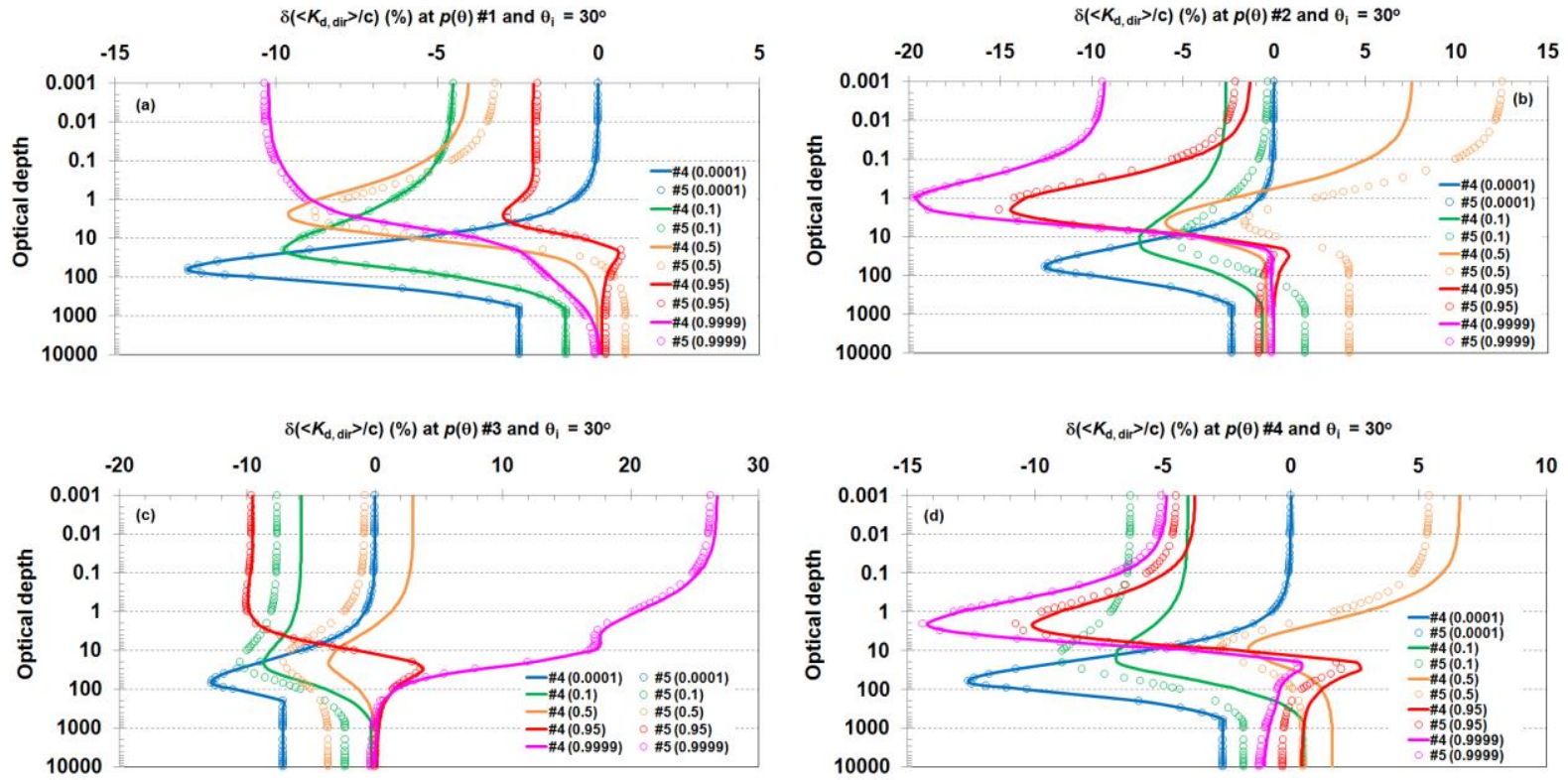
Fig. 14. The same as Fig. 13, but for $\theta_{i}=30^{\circ}$.
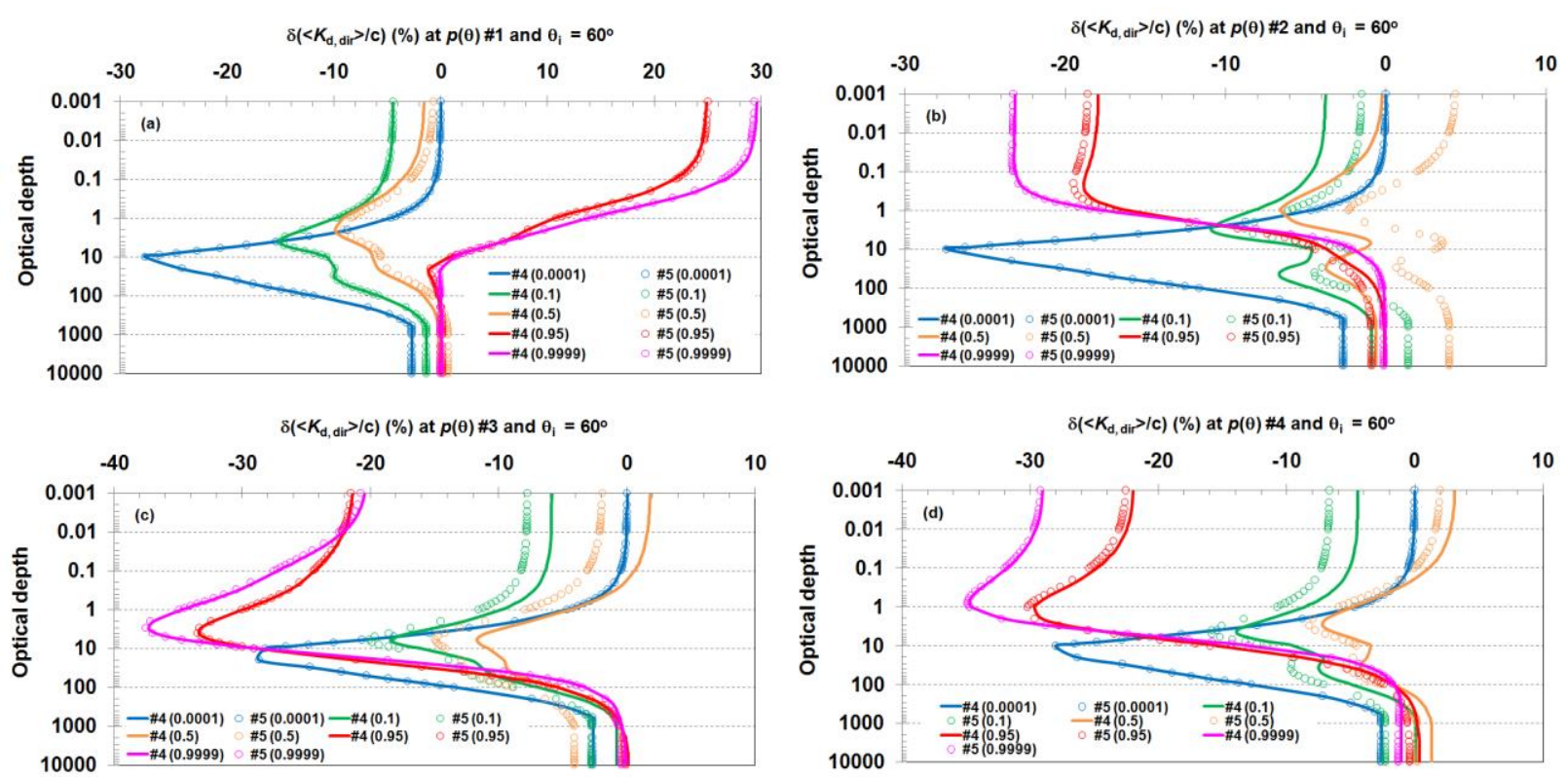

Fig. 15. The same as Fig. 13, but for $\theta i=60^{\circ}$.

Table 7. Plane transmittance by layer for normal light incidence as a function of optical depth $\tau$ asymmetry parameter $g$, and single-scattering albedo $\omega_{0}$. Position of the numbers in cells, top-to-bottom, corresponds to the computations performed by IPOL, adding-doubling method, and HKS 2 approximation (\#4 in Table 6), respectively.

\begin{tabular}{ccccc}
\hline & \multicolumn{5}{c}{$\boldsymbol{g = 0}$} \\
\hline$\tau / \omega_{0}$ & $\mathbf{0 . 2}$ & $\mathbf{0 . 4}$ & $\mathbf{0 . 6}$ & $\mathbf{0 . 8}$ \\
\hline \multirow{2}{*}{$\mathbf{2}^{-15}$} & 0.99997253 & 0.99997559 & 0.99997864 & 0.99998169 \\
& 1.0000 & 1.0000 & 1.0000 & 1.0000 \\
& 0.9999743 & 0.9999770 & 0.9999792 & 0.9999818 \\
& 0.99994507 & 0.99995117 & 0.99995728 & 0.99996338 \\
$\mathbf{2}^{-14}$ & 0.9999 & 0.9999 & 1.0000 & 1.0000 \\
& 0.9999486 & 0.9999540 & 0.9999584 & 0.9999635 \\
$\mathbf{2}^{-13}$ & 0.99989014 & 0.99990235 & 0.99991456 & 0.99992677 \\
& 0.9999 & 0.9999 & 0.9999 & 0.9999
\end{tabular}




\begin{tabular}{|c|c|c|c|c|}
\hline & 0.9998972 & 0.9999080 & 0.9999168 & 0.9999271 \\
\hline $2^{-12}$ & $\begin{array}{c}0.99978029 \\
0.9998 \\
0.999794\end{array}$ & $\begin{array}{c}0.99980470 \\
0.9998 \\
0.999816\end{array}$ & $\begin{array}{c}0.99982911 \\
0.9998 \\
0.999834\end{array}$ & $\begin{array}{c}0.99985354 \\
0.9999 \\
0.999854\end{array}$ \\
\hline $2^{-11}$ & $\begin{array}{c}0.99956061 \\
0.9996 \\
0.999589\end{array}$ & $\begin{array}{c}0.99960941 \\
0.9996 \\
0.999632\end{array}$ & $\begin{array}{c}0.99965823 \\
0.9997 \\
0.999667\end{array}$ & $\begin{array}{c}0.99970708 \\
0.9997 \\
0.999708\end{array}$ \\
\hline $2^{-10}$ & $\begin{array}{c}0.99912133 \\
0.9991 \\
0.999178\end{array}$ & $\begin{array}{c}0.99921885 \\
0.9992 \\
0.999264\end{array}$ & $\begin{array}{c}0.99931647 \\
0.9993 \\
0.999334\end{array}$ & $\begin{array}{c}0.99941420 \\
0.9994 \\
0.999417\end{array}$ \\
\hline $2^{-9}$ & $\begin{array}{c}0.99824312 \\
0.9982 \\
0.99836\end{array}$ & $\begin{array}{c}0.99843786 \\
0.9984 \\
0.99853\end{array}$ & $\begin{array}{c}0.99863301 \\
0.9986 \\
0.99867\end{array}$ & $\begin{array}{c}0.99882857 \\
0.9988 \\
0.99883\end{array}$ \\
\hline $2^{-8}$ & $\begin{array}{c}0.99648808 \\
0.9965 \\
0.99672\end{array}$ & $\begin{array}{c}0.99687638 \\
0.9969 \\
0.99706\end{array}$ & $\begin{array}{c}0.99726629 \\
0.9973 \\
0.99734\end{array}$ & $\begin{array}{c}0.99765782 \\
0.9977 \\
0.99767\end{array}$ \\
\hline $2^{-7}$ & $\begin{array}{c}0.99298381 \\
0.9930 \\
0.99344\end{array}$ & $\begin{array}{c}0.99375582 \\
0.9938 \\
0.99413\end{array}$ & $\begin{array}{c}0.99453405 \\
0.9945 \\
0.99469\end{array}$ & $\begin{array}{c}0.99531857 \\
0.9953 \\
0.99534\end{array}$ \\
\hline $2^{-6}$ & $\begin{array}{c}0.98600000 \\
0.9860 \\
0.9869\end{array}$ & $\begin{array}{c}0.98752647 \\
0.9875 \\
0.9883\end{array}$ & $\begin{array}{c}0.98907637 \\
0.9891 \\
0.9894\end{array}$ & $\begin{array}{c}0.99065024 \\
0.9906 \\
0.9907\end{array}$ \\
\hline $2^{-5}$ & $\begin{array}{c}0.97214014 \\
0.9721 \\
0.9740\end{array}$ & $\begin{array}{c}0.97512811 \\
0.9751 \\
0.9767\end{array}$ & $\begin{array}{c}0.97820061 \\
0.9782 \\
0.9789\end{array}$ & $\begin{array}{c}0.98136127 \\
0.9814 \\
0.9815\end{array}$ \\
\hline $2^{-4}$ & $\begin{array}{c}0.94488885 \\
0.9449 \\
0.9487\end{array}$ & $\begin{array}{c}0.95063215 \\
0.9506 \\
0.9539\end{array}$ & $\begin{array}{c}0.95666311 \\
0.9567 \\
0.9582\end{array}$ & $\begin{array}{c}0.96300395 \\
0.9630 \\
0.9634\end{array}$ \\
\hline $2^{-3}$ & $\begin{array}{c}0.89235095 \\
0.8924 \\
0.9000\end{array}$ & $\begin{array}{c}0.90301916 \\
0.9030 \\
0.9099\end{array}$ & $\begin{array}{c}0.91460706 \\
0.9146 \\
0.9182\end{array}$ & $\begin{array}{c}0.92723927 \\
0.9272 \\
0.9283\end{array}$ \\
\hline $2^{-2}$ & $\begin{array}{c}0.79509490 \\
0.7951 \\
0.8097\end{array}$ & $\begin{array}{c}0.81361713 \\
0.8136 \\
0.8276\end{array}$ & $\begin{array}{c}0.83485819 \\
0.8348 \\
0.8428\end{array}$ & $\begin{array}{c}0.85946419 \\
0.8595 \\
0.8621\end{array}$ \\
\hline 0.5 & $\begin{array}{c}0.62957661 \\
0.6296 \\
0.6548\end{array}$ & $\begin{array}{c}0.65770904 \\
0.6577 \\
0.6835\end{array}$ & $\begin{array}{c}0.6928068 \\
0.6928 \\
0.7093\end{array}$ & $\begin{array}{c}0.73779878 \\
0.7378 \\
0.7442\end{array}$ \\
\hline 1 & $\begin{array}{c}0.39226229 \\
0.3923 \\
0.4265\end{array}$ & $\begin{array}{c}0.42506227 \\
0.4251 \\
0.4633\end{array}$ & $\begin{array}{c}0.47138775 \\
0.4714 \\
0.4994\end{array}$ & $\begin{array}{c}0.5414205 \\
0.5414 \\
0.5548\end{array}$ \\
\hline
\end{tabular}




\begin{tabular}{|c|c|c|c|c|}
\hline \multirow{4}{*}{2} & & & & \\
\hline & 0.15024257 & 0.1729701 & 0.21114949 & 0.28596783 \\
\hline & 0.1502 & 0.1730 & 0.2111 & 0.2859 \\
\hline & 0.1782 & 0.2072 & 0.2405 & 0.3050 \\
\hline \multirow{3}{*}{4} & 0.021502064 & 0.027199569 & 0.039376747 & 0.075093197 \\
\hline & 0.0215 & 0.0272 & 0.0394 & 0.0751 \\
\hline & 0.0294 & 0.0374 & 0.0496 & 0.0851 \\
\hline \multirow{3}{*}{8} & 4.2338879E-4 & $6.1694335 \mathrm{E}-4$ & 1.2085188E-3 & 4.6482094E-3 \\
\hline & 0.0004 & 0.0006 & 0.0012 & 0.0046 \\
\hline & $6.7 E-4$ & $9.0 \mathrm{E}-4$ & $1.5 \mathrm{E}-3$ & $5.1 \mathrm{E}-3$ \\
\hline \multirow{3}{*}{16} & $1.5524709 \mathrm{E}-7$ & $2.7941926 \mathrm{E}-7$ & 9.6014032E-7 & 1.6134489E-5 \\
\hline & 0.0000 & 0.0000 & 0.0000 & 0.0000 \\
\hline & 2.3E-7 & $3.0 \mathrm{E}-7$ & 8.7E-7 & $1.4 \mathrm{E}-5$ \\
\hline \multirow{3}{*}{32} & $1.9418148 \mathrm{E}-14$ & 4.8259663E-14 & $5.0738270 \mathrm{E}-13$ & $1.8718950 \mathrm{E}-10$ \\
\hline & 0.0000 & 0.0000 & 0.0000 & 0.0000 \\
\hline & 1.8E-14 & 3.0E-14 & $3.6 \mathrm{E}-13$ & $1.5 \mathrm{E}-10$ \\
\hline \multirow{3}{*}{64} & $2.7777169 \mathrm{E}-28$ & 1.1602353E-27 & 1.2652846E-25 & 2.5105928E-20 \\
\hline & 0.0000 & 0.0000 & 0.0000 & 0.0000 \\
\hline & 1.7E-28 & $6.5 E-28$ & $1.1 \mathrm{E}-25$ & $2.4 \mathrm{E}-20$ \\
\hline \multirow{3}{*}{128} & $5.0214676 \mathrm{E}-56$ & $5.1415276 E-55$ & $7.6468406 \mathrm{E}-51$ & $4.5159871 \mathrm{E}-40$ \\
\hline & 0.0000 & 0.0000 & 0.0000 & 0.0000 \\
\hline & 1.3904711E-111 & $7.5295881 \mathrm{E}-110$ & $2.7889464 \mathrm{E}-101$ & 1.4611861E-79 \\
\hline \multirow[t]{2}{*}{256} & 0.0000 & 0.0000 & 0.0000 & 0.0000 \\
\hline & 7.6E-112 & 1.8E-109 & $1.9 E-100$ & $4.0 \mathrm{E}-79$ \\
\hline \multirow{3}{*}{512} & 9.3708033E-223 & 1.3270825E-219 & 3.7098365E-202 & $1.5297174 \mathrm{E}-158$ \\
\hline & 0.0000 & 0.0000 & 0.0000 & 0.0000 \\
\hline & 5.8E-223 & 3.1E-218 & 3.6E-200 & 1.7E-157 \\
\hline \multicolumn{5}{|c|}{$g=0$} \\
\hline$\tau / \omega 0$ & 0.9 & 0.95 & 0.99 & 1 \\
\hline \multirow{3}{*}{$2^{-15}$} & 0.99998322 & 0.99998398 & 0.99998459 & 0.99998474 \\
\hline & 1.0000 & 1.0000 & 1.0000 & 1.0000 \\
\hline & 0.9999836 & 0.9999849 & 0.9999865 & 0.9999874 \\
\hline \multirow{3}{*}{$2^{-14}$} & 0.99996644 & 0.99996796 & 0.99996918 & 0.99996949 \\
\hline & 1.0000 & 1.0000 & 1.0000 & 1.0000 \\
\hline & 0.9999673 & 0.9999699 & 0.9999730 & 0.9999748 \\
\hline \multirow{3}{*}{$2^{-13}$} & 0.99993287 & 0.99993592 & 0.99993837 & 0.99993898 \\
\hline & 0.9999 & 0.9999 & 0.9999 & 0.9999 \\
\hline & 0.9999346 & 0.9999398 & 0.9999459 & 0.9999495 \\
\hline \multirow{3}{*}{$2^{-12}$} & 0.99986575 & 0.99987186 & 0.99987674 & 0.99987796 \\
\hline & 0.9999 & 0.9999 & 0.9999 & 0.9999 \\
\hline & 0.999869 & 0.999880 & 0.999892 & 0.999899 \\
\hline $2^{-11}$ & 0.99973151 & 0.99974373 & 0.99975351 & 0.99975596 \\
\hline
\end{tabular}




\begin{tabular}{|c|c|c|c|c|}
\hline & $\begin{array}{c}0.9997 \\
0.999738\end{array}$ & $\begin{array}{c}0.9997 \\
0.999759\end{array}$ & $\begin{array}{c}0.9998 \\
0.999784\end{array}$ & $\begin{array}{c}0.9998 \\
0.999798\end{array}$ \\
\hline $2^{-10}$ & $\begin{array}{c}0.99946310 \\
0.9995 \\
0.999477\end{array}$ & $\begin{array}{c}0.99948756 \\
0.9995 \\
0.999518\end{array}$ & $\begin{array}{c}0.99950714 \\
0.9995 \\
0.999567\end{array}$ & $\begin{array}{c}0.99951203 \\
0.9995 \\
0.999596\end{array}$ \\
\hline $2^{-9}$ & $\begin{array}{c}0.99892650 \\
0.9989 \\
0.99895\end{array}$ & $\begin{array}{c}0.99897550 \\
0.9990 \\
0.99904\end{array}$ & $\begin{array}{c}0.99901473 \\
0.9990 \\
0.99914\end{array}$ & $\begin{array}{c}0.99902453 \\
0.9990 \\
0.99919\end{array}$ \\
\hline $2^{-8}$ & $\begin{array}{c}0.99785419 \\
0.9979 \\
0.99791\end{array}$ & $\begin{array}{c}0.99795252 \\
0.9980 \\
0.99808\end{array}$ & $\begin{array}{c}0.99803127 \\
0.9980 \\
0.99827\end{array}$ & $\begin{array}{c}0.99805096 \\
0.9981 \\
0.99839\end{array}$ \\
\hline $2^{-7}$ & $\begin{array}{c}0.99571321 \\
0.9957 \\
0.99582\end{array}$ & $\begin{array}{c}0.99591113 \\
0.9959 \\
0.99616\end{array}$ & $\begin{array}{c}0.99606975 \\
0.9961 \\
0.99655\end{array}$ & $\begin{array}{c}0.99610945 \\
0.9961 \\
0.99678\end{array}$ \\
\hline $2^{-6}$ & $\begin{array}{c}0.99144634 \\
0.9914 \\
0.9917\end{array}$ & $\begin{array}{c}0.99184672 \\
0.9918 \\
0.9923\end{array}$ & $\begin{array}{c}0.99216814 \\
0.9921 \\
0.9931\end{array}$ & $\begin{array}{c}0.99224865 \\
0.9922 \\
0.9936\end{array}$ \\
\hline $2^{-5}$ & $\begin{array}{c}0.98297585 \\
0.9830 \\
0.9834\end{array}$ & $\begin{array}{c}0.98379193 \\
0.9838 \\
0.9848\end{array}$ & $\begin{array}{c}0.98444906 \\
0.9844 \\
0.9863\end{array}$ & $\begin{array}{c}0.98461394 \\
0.9846 \\
0.9872\end{array}$ \\
\hline $2^{-4}$ & $\begin{array}{c}0.96629816 \\
0.9663 \\
0.9672\end{array}$ & $\begin{array}{c}0.96797764 \\
0.9680 \\
0.9698\end{array}$ & $\begin{array}{c}0.96933714 \\
0.9693 \\
0.9729\end{array}$ & $\begin{array}{c}0.96967926 \\
0.9697 \\
0.9747\end{array}$ \\
\hline $2^{-3}$ & $\begin{array}{c}0.93399223 \\
0.9340 \\
0.9358\end{array}$ & $\begin{array}{c}0.93748685 \\
0.9375 \\
0.9410\end{array}$ & $\begin{array}{c}0.94034180 \\
0.9403 \\
0.9471\end{array}$ & $\begin{array}{c}0.94106397 \\
0.9411 \\
0.9506\end{array}$ \\
\hline $2^{-2}$ & $\begin{array}{c}0.87328918 \\
0.8733 \\
0.8767\end{array}$ & $\begin{array}{c}0.88063824 \\
0.8806 \\
0.8869\end{array}$ & $\begin{array}{c}0.88674408 \\
0.8867 \\
0.8988\end{array}$ & $\begin{array}{c}0.88830336 \\
0.8883 \\
0.9054\end{array}$ \\
\hline 0.5 & $\begin{array}{c}0.76540120 \\
0.7654 \\
0.7716\end{array}$ & $\begin{array}{c}0.78082807 \\
0.7808 \\
0.7911\end{array}$ & $\begin{array}{c}0.79406935 \\
0.7940 \\
0.8136\end{array}$ & $\begin{array}{c}0.79751479 \\
0.7975 \\
0.8255\end{array}$ \\
\hline 1 & $\begin{array}{c}0.59164929 \\
0.5916 \\
0.6025\end{array}$ & $\begin{array}{c}0.62259306 \\
0.6226 \\
0.6375\end{array}$ & $\begin{array}{c}0.65100478 \\
0.6510 \\
0.6784\end{array}$ & $\begin{array}{c}0.65869879 \\
0.6587 \\
0.6986\end{array}$ \\
\hline 2 & $\begin{array}{c}0.35652379 \\
0.3565 \\
0.3720\end{array}$ & $\begin{array}{c}0.40933412 \\
0.4093 \\
0.4273\end{array}$ & $\begin{array}{c}0.46572698 \\
0.4657 \\
0.4959\end{array}$ & $\begin{array}{c}0.48251337 \\
0.4825 \\
0.5276\end{array}$ \\
\hline 4 & $\begin{array}{c}0.12853767 \\
0.1285\end{array}$ & $\begin{array}{c}0.18693361 \\
0.1869\end{array}$ & $\begin{array}{c}0.27550228 \\
0.2755\end{array}$ & $\begin{array}{c}0.30909669 \\
0.3091\end{array}$ \\
\hline
\end{tabular}




\begin{tabular}{|c|c|c|c|c|}
\hline & 0.1395 & 0.2002 & 0.2978 & 0.3447 \\
\hline 8 & $\begin{array}{c}0.016022787 \\
0.0160 \\
0.0171\end{array}$ & $\begin{array}{c}0.040767504 \\
0.0408 \\
0.0430\end{array}$ & $\begin{array}{c}0.12208116 \\
0.1221 \\
0.1299\end{array}$ & $\begin{array}{c}0.17821187 \\
0.1782 \\
0.1962\end{array}$ \\
\hline 16 & $\begin{array}{c}2.402488 \mathrm{E}-4 \\
0.0002 \\
2.2 \mathrm{E}-4\end{array}$ & $\begin{array}{c}1.9584513 \mathrm{E}-3 \\
0.0020 \\
1.8 \mathrm{E}-3\end{array}$ & $\begin{array}{c}0.029602855 \\
0.0296 \\
0.0298\end{array}$ & $\begin{array}{c}0.096377248 \\
0.0964 \\
0.1033\end{array}$ \\
\hline 32 & $\begin{array}{c}5.3677625 \mathrm{E}-8 \\
0.0000 \\
4.5 \mathrm{E}-8\end{array}$ & $\begin{array}{c}4.5196595 \mathrm{E}-6 \\
0.0000 \\
4.0 \mathrm{E}-6\end{array}$ & $\begin{array}{c}1.8689861 \mathrm{E}-3 \\
0.0019 \\
1.8 \mathrm{E}-13\end{array}$ & $\begin{array}{c}0.050237361 \\
0.0502 \\
0.0448\end{array}$ \\
\hline 64 & $\begin{array}{c}2.6791877 \mathrm{E}-15 \\
0.0000 \\
2.4 \mathrm{E}-15\end{array}$ & $\begin{array}{c}2.4070728 \mathrm{E}-11 \\
0.0000 \\
2.2 \mathrm{E}-11\end{array}$ & $\begin{array}{c}\text { 7.4861946E-6 } \\
0.0000 \\
7.3 E-6\end{array}$ & $\begin{array}{c}0.025663887 \\
0.0257 \\
0.0275\end{array}$ \\
\hline 128 & $\begin{array}{c}6.6745624 \mathrm{E}-30 \\
0.0000 \\
6.8 \mathrm{E}-30\end{array}$ & $\begin{array}{c}6.8274138 \mathrm{E}-22 \\
0.0000 \\
6.4 \mathrm{E}-22\end{array}$ & $\begin{array}{c}1.2011014 \mathrm{E}-10 \\
0.0000 \\
1.2 \mathrm{E}-10\end{array}$ & $\begin{array}{c}0.012972029 \\
0.0130 \\
0.0140\end{array}$ \\
\hline 256 & $\begin{array}{c}4.1424963 \mathrm{E}-59 \\
0.0000 \\
5.6 \mathrm{E}-59\end{array}$ & $\begin{array}{c}5.4927549 \mathrm{E}-43 \\
0.0000 \\
5.8 \mathrm{E}-43\end{array}$ & $\begin{array}{c}3.0918387 \mathrm{E}-20 \\
0.0000 \\
3.0 \mathrm{E}-20\end{array}$ & $\begin{array}{c}6.5202049 \mathrm{E}-3 \\
0.0065 \\
7.0 \mathrm{E}-3\end{array}$ \\
\hline 512 & $\begin{array}{c}1.5956616 \mathrm{E}-117 \\
0.0000 \\
3.8 \mathrm{E}-117\end{array}$ & $\begin{array}{c}3.5551524 \mathrm{E}-85 \\
0.0000 \\
4.6 \mathrm{E}-85\end{array}$ & $\begin{array}{c}2.0487601 \mathrm{E}-39 \\
0.0000 \\
2.0 \mathrm{E}-39\end{array}$ & $\begin{array}{c}3.2659031 \mathrm{E}-3 \\
0.0033 \\
3.5 \mathrm{E}-3\end{array}$ \\
\hline \multicolumn{5}{|c|}{$g=0.5$} \\
\hline$\tau / \omega 0$ & 0.2 & 0.4 & 0.6 & 0.8 \\
\hline $2^{-15}$ & $\begin{array}{c}0.99997454 \\
- \\
0.9999749\end{array}$ & $\begin{array}{c}0.99997960 \\
- \\
0.9999786\end{array}$ & $\begin{array}{c}0.99998467 \\
1.0000 \\
0.9999829\end{array}$ & $\begin{array}{c}0.99998973 \\
- \\
0.9999886\end{array}$ \\
\hline $2^{-14}$ & $\begin{array}{c}0.99994909 \\
- \\
0.9999498\end{array}$ & $\begin{array}{c}0.99995921 \\
- \\
0.9999572\end{array}$ & $\begin{array}{c}0.99996933 \\
1.0000 \\
0.9999657\end{array}$ & $\begin{array}{c}0.99997945 \\
- \\
0.9999772\end{array}$ \\
\hline $2^{-13}$ & $\begin{array}{c}0.99989818 \\
- \\
0.9998997\end{array}$ & $\begin{array}{c}0.99991842 \\
- \\
0.9999144\end{array}$ & $\begin{array}{c}0.99993866 \\
0.9999 \\
0.9999314\end{array}$ & $\begin{array}{c}0.99995890 \\
- \\
0.9999543\end{array}$ \\
\hline $2^{-12}$ & $\begin{array}{c}0.99979636 \\
- \\
0.999799\end{array}$ & $\begin{array}{c}0.99983684 \\
- \\
0.999829\end{array}$ & $\begin{array}{c}0.99987732 \\
0.9999 \\
0.999863\end{array}$ & $\begin{array}{c}0.99991780 \\
- \\
0.999909\end{array}$ \\
\hline $2^{-11}$ & $\begin{array}{c}0.99959275 \\
- \\
0.999599\end{array}$ & $\begin{array}{c}0.99967368 \\
- \\
0.999657\end{array}$ & $\begin{array}{c}0.99975462 \\
0.9998 \\
0.999726\end{array}$ & $\begin{array}{c}0.99983559 \\
- \\
0.999817\end{array}$ \\
\hline $2^{-10}$ & 0.99918560 & 0.99934737 & 0.99950921 & 0.99967113 \\
\hline
\end{tabular}




\begin{tabular}{|c|c|c|c|c|}
\hline & 0 & & 0.9995 & \\
\hline & 0.999198 & 0.999315 & 0.999451 & 0.999634 \\
\hline $2^{-9}$ & $\begin{array}{c}0.99837165 \\
- \\
0.99840\end{array}$ & $\begin{array}{c}0.99869482 \\
- \\
0.99863\end{array}$ & $\begin{array}{c}0.99901829 \\
0.9990 \\
0.99890\end{array}$ & $\begin{array}{c}0.99934207 \\
- \\
0.99927\end{array}$ \\
\hline $2^{-8}$ & $\begin{array}{c}0.99674508 \\
- \\
0.99680\end{array}$ & $\begin{array}{c}0.99738997 \\
- \\
0.99726\end{array}$ & $\begin{array}{c}0.99803607 \\
0.9980 \\
0.99781\end{array}$ & $\begin{array}{c}0.99868337 \\
- \\
0.99854\end{array}$ \\
\hline $2^{-7}$ & $\begin{array}{c}0.99349741 \\
- \\
0.9936\end{array}$ & $\begin{array}{c}0.99478151 \\
- \\
0.9945\end{array}$ & $\begin{array}{c}0.99607030 \\
0.9961 \\
0.9956\end{array}$ & $\begin{array}{c}0.99736383 \\
- \\
0.9971\end{array}$ \\
\hline $2^{-6}$ & $\begin{array}{c}0.98702484 \\
- \\
0.9872\end{array}$ & $\begin{array}{c}0.98957082 \\
- \\
0.9891\end{array}$ & $\begin{array}{c}0.99213477 \\
0.9921 \\
0.9913\end{array}$ & $\begin{array}{c}0.99471708 \\
- \\
0.9942\end{array}$ \\
\hline $2^{-5}$ & $\begin{array}{c}0.97417566 \\
- \\
0.9746\end{array}$ & $\begin{array}{c}0.97918235 \\
- \\
0.9783\end{array}$ & $\begin{array}{c}0.98425581 \\
0.9843 \\
0.9826\end{array}$ & $\begin{array}{c}0.98939870 \\
- \\
0.9884\end{array}$ \\
\hline $2^{-4}$ & $\begin{array}{c}0.94888224 \\
- \\
0.9499\end{array}$ & $\begin{array}{c}0.95857497 \\
- \\
0.9570\end{array}$ & $\begin{array}{c}0.96850597 \\
0.9685 \\
0.9654\end{array}$ & $\begin{array}{c}0.97869161 \\
- \\
0.9768\end{array}$ \\
\hline $2^{-3}$ & $\begin{array}{c}0.89996643 \\
- \\
0.9023\end{array}$ & $\begin{array}{c}0.91817097 \\
- \\
0.9158\end{array}$ & $\begin{array}{c}0.93718895 \\
0.9372 \\
0.9319\end{array}$ & $\begin{array}{c}0.95711380 \\
- \\
0.9541\end{array}$ \\
\hline $2^{-2}$ & $\begin{array}{c}0.80877912 \\
- \\
0.8138\end{array}$ & $\begin{array}{c}0.84099202 \\
- \\
0.8384\end{array}$ & $\begin{array}{c}0.87582039 \\
0.8758 \\
0.8681\end{array}$ & $\begin{array}{c}0.91376447 \\
- \\
0.9098\end{array}$ \\
\hline 0.5 & $\begin{array}{c}0.65132128 \\
- \\
0.6616\end{array}$ & $\begin{array}{c}0.70201261 \\
- \\
0.7017\end{array}$ & $\begin{array}{c}0.76020392 \\
0.7602 \\
0.7520\end{array}$ & $\begin{array}{c}0.82830799 \\
- \\
0.8261\end{array}$ \\
\hline 1 & $\begin{array}{c}0.41916822 \\
- \\
0.4357\end{array}$ & $\begin{array}{c}0.4824385 \\
- \\
0.4890\end{array}$ & $\begin{array}{c}0.56289917 \\
0.5629 \\
0.5609\end{array}$ & $\begin{array}{c}0.67004078 \\
- \\
0.6772\end{array}$ \\
\hline 2 & $\begin{array}{c}0.17038876 \\
- \\
0.1866\end{array}$ & $\begin{array}{c}0.22020607 \\
- \\
0.2329\end{array}$ & $\begin{array}{c}0.29548964 \\
0.2955 \\
0.3047\end{array}$ & $\begin{array}{c}0.42147850 \\
- \\
0.4447\end{array}$ \\
\hline 4 & $\begin{array}{c}0.027039146 \\
- \\
0.0326\end{array}$ & $\begin{array}{c}0.042668678 \\
- \\
0.0492\end{array}$ & $\begin{array}{c}0.074352914 \\
0.0744 \\
0.0826\end{array}$ & $\begin{array}{c}0.15388166 \\
- \\
0.1758\end{array}$ \\
\hline 8 & 6.3096939E-4 & 1.4080573E-3 & $\begin{array}{c}4.0698841 \mathrm{E}-3 \\
0.0041\end{array}$ & 0.018359019 \\
\hline
\end{tabular}


8.5E-4

3.0328924E-7

16

$3.9 \mathrm{E}-7$

5.8654054E-14

32

64

$$
\text { 4.7E-14 }
$$

1.7557793E-27

7.6E-28

$1.2275246 \mathrm{E}-54$

128

256

512

$$
\text { 4.6E-55 }
$$

4.9170644E-109

2.1E-109

7.3719268E-218
$1.8 \mathrm{E}-3$

1.275935E-6

1.4E-6

8.6863526E-13

5.8E-13

3.6249777E-25

$1.8 \mathrm{E}-25$

6.188342E-50

2.9E-50

1.8024131E-99

8.5E-100
4.7E-3

$1.0443475 \mathrm{E}-5$

0.0000

9.9E-6

6.3401196E-11

0.0000

4.1E-11

2.3079825E-21

0.0000

1.3E-21

3.0577749E-42

0.0000

1.7E-42

5.3672459E-84

0.0000

3.1E-84
0.0213

2.4248186E-4

2.2E-4

4.1660606E-8

3.0E-8

$1.2292318 \mathrm{E}-15$

8.5E-16

1.0701634E-30

7.4E-31

8.1111555E-61

5.6E-61

4.6595928E-121 0.0000

9.5E-168

$$
g=0.5
$$

\begin{tabular}{ccccc}
\hline$\tau / \omega_{0}$ & $\mathbf{0 . 9}$ & $\mathbf{0 . 9 5}$ & $\mathbf{0 . 9 9}$ & $\mathbf{1}$ \\
\hline \multirow{2}{*}{$\mathbf{2}^{-15}$} & 0.99999226 & 0.99999352 & 0.99999453 & 0.99999479 \\
& 1.0000 & - & 1.0000 & - \\
& 0.9999918 & 0.9999935 & 0.9999949 & 0.9999999 \\
$\mathbf{2}^{-14}$ & 0.99998451 & 0.99998704 & 0.99998907 & 0.99998957 \\
& 1.0000 & - & 1.0000 & - \\
& 0.9999837 & 0.9999870 & 0.9999897 & 0.9999999 \\
$\mathbf{2}^{-13}$ & 0.99996902 & 0.99997409 & 0.99997813 & 0.99997915 \\
& 1.0000 & - & 1.0000 & - \\
& 0.9999673 & 0.9999740 & 0.9999795 & 0.9999998 \\
$\mathbf{2}^{-12}$ & 0.99993805 & 0.99994817 & 0.99995627 & 0.99995829 \\
& 0.9999 & - & 1.0000 & - \\
& 0.999935 & 0.999948 & 0.999959 & 0.9999996 \\
$\mathbf{2}^{-11}$ & 0.99987608 & 0.99989633 & 0.99991253 & 0.99991658 \\
& 0.9999 & - & 0.9999 & - \\
& 0.999869 & 0.999896 & 0.999918 & 0.9999991 \\
$\mathbf{2}^{-10}$ & 0.99975212 & 0.99979262 & 0.99982503 & 0.99983313 \\
& 0.9998 & - & 0.9998 & - \\
\multirow{2}{*}{$\mathbf{2}^{-9}$} & 0.999738 & 0.999792 & 0.999836 & 0.999998 \\
& 0.99950407 & 0.99958510 & 0.99964994 & 0.99966615 \\
& 0.9995 & - & 0.9996 & -
\end{tabular}




\begin{tabular}{|c|c|c|c|c|}
\hline & 0.99948 & 0.99958 & 0.99967 & 0.999996 \\
\hline $2^{-8}$ & $\begin{array}{c}0.99900748 \\
0.9990 \\
0.99895\end{array}$ & $\begin{array}{c}0.99916964 \\
- \\
0.99917\end{array}$ & $\begin{array}{c}0.99929943 \\
0.9993 \\
0.99934\end{array}$ & $\begin{array}{c}0.99933189 \\
- \\
0.999993\end{array}$ \\
\hline $2^{-7}$ & $\begin{array}{c}0.99801239 \\
0.9980 \\
0.9979\end{array}$ & $\begin{array}{c}0.99833712 \\
- \\
0.9983\end{array}$ & $\begin{array}{c}0.99859712 \\
0.9986 \\
0.9987\end{array}$ & $\begin{array}{c}0.99866215 \\
- \\
0.999986\end{array}$ \\
\hline $2^{-6}$ & $\begin{array}{c}0.99601524 \\
0.9960 \\
0.9958\end{array}$ & $\begin{array}{c}0.99666610 \\
- \\
0.9967\end{array}$ & $\begin{array}{c}0.99718765 \\
0.9972 \\
0.9974\end{array}$ & $\begin{array}{c}0.99731815 \\
- \\
0.999971\end{array}$ \\
\hline $2^{-5}$ & $\begin{array}{c}0.99199705 \\
0.9820 \\
0.9817\end{array}$ & $\begin{array}{c}0.99330312 \\
- \\
0.9933\end{array}$ & $\begin{array}{c}0.99435132 \\
0.9944 \\
0.9948\end{array}$ & $\begin{array}{c}0.99461384 \\
- \\
0.999943\end{array}$ \\
\hline $2^{-4}$ & $\begin{array}{c}0.98388556 \\
0.9839 \\
0.9834\end{array}$ & $\begin{array}{c}0.98650889 \\
- \\
0.9867\end{array}$ & $\begin{array}{c}0.98862050 \\
0.9886 \\
0.9895\end{array}$ & $\begin{array}{c}0.98915022 \\
- \\
0.99989\end{array}$ \\
\hline $2^{-3}$ & $\begin{array}{c}0.96745054 \\
0.9675 \\
0.9669\end{array}$ & $\begin{array}{c}0.97271931 \\
- \\
0.9736\end{array}$ & $\begin{array}{c}0.97698447 \\
0.9770 \\
0.9791\end{array}$ & $\begin{array}{c}0.97805788 \\
- \\
0.99977\end{array}$ \\
\hline $2^{-2}$ & $\begin{array}{c}0.93410667 \\
0.9341 \\
0.9346\end{array}$ & $\begin{array}{c}0.94466395 \\
- \\
0.9476\end{array}$ & $\begin{array}{c}0.95330846 \\
0.9533 \\
0.9586\end{array}$ & $\begin{array}{c}0.95549825 \\
- \\
0.99953\end{array}$ \\
\hline 0.5 & $\begin{array}{c}0.86720208 \\
0.8672 \\
0.8723\end{array}$ & $\begin{array}{c}0.88813706 \\
- \\
0.8972\end{array}$ & $\begin{array}{c}0.90569347 \\
0.9057 \\
0.9187\end{array}$ & $\begin{array}{c}0.91020283 \\
- \\
0.99905\end{array}$ \\
\hline 1 & $\begin{array}{c}0.73909114 \\
0.7391 \\
0.7567\end{array}$ & $\begin{array}{c}0.77914519 \\
- \\
0.8022\end{array}$ & $\begin{array}{c}0.81451629 \\
0.8145 \\
0.8432\end{array}$ & $\begin{array}{c}0.82388501 \\
- \\
0.9980\end{array}$ \\
\hline 2 & $\begin{array}{c}0.52327333 \\
0.5233 \\
0.5602\end{array}$ & $\begin{array}{c}0.59210338 \\
- \\
0.6356\end{array}$ & $\begin{array}{c}0.66030018 \\
0.6603 \\
0.7096\end{array}$ & $\begin{array}{c}0.67969982 \\
- \\
0.9957\end{array}$ \\
\hline 4 & $\begin{array}{c}0.25053246 \\
0.2505 \\
0.2884\end{array}$ & $\begin{array}{c}0.33904538 \\
- \\
0.3859\end{array}$ & $\begin{array}{c}0.45265050 \\
0.4527 \\
0.5052\end{array}$ & $\begin{array}{c}0.49103334 \\
- \\
0.9904\end{array}$ \\
\hline 8 & $\begin{array}{c}0.054391556 \\
0.0544 \\
0.0630\end{array}$ & $\begin{array}{c}0.11250104 \\
- \\
0.1278\end{array}$ & $\begin{array}{c}0.24383127 \\
0.2438 \\
0.2674\end{array}$ & $\begin{array}{c}0.31102747 \\
- \\
0.9776\end{array}$ \\
\hline 16 & $\begin{array}{c}2.4917786 \mathrm{E}-3 \\
0.0025 \\
2.3 \mathrm{E}-3\end{array}$ & $\begin{array}{c}0.01260914 \\
- \\
0.0122\end{array}$ & $\begin{array}{c}0.086018839 \\
0.0860 \\
0.0859\end{array}$ & $\begin{array}{c}0.17902418 \\
- \\
0.9499\end{array}$ \\
\hline
\end{tabular}




\begin{tabular}{|c|c|c|c|c|}
\hline 32 & $\begin{array}{c}5.2117601 \mathrm{E}-6 \\
0.0000 \\
4.1 \mathrm{E}-6\end{array}$ & $\begin{array}{c}1.5897942 \mathrm{E}-4 \\
- \\
1.4 \mathrm{E}-14\end{array}$ & $\begin{array}{c}0.012012305 \\
0.0120 \\
0.0112\end{array}$ & $\begin{array}{c}0.09682697 \\
- \\
0.9008\end{array}$ \\
\hline 64 & $\begin{array}{c}2.2799263 \mathrm{E}-11 \\
0.0000 \\
1.8 \mathrm{E}-11\end{array}$ & $\begin{array}{c}2.5274213 \mathrm{E}-8 \\
- \\
2.1 \mathrm{E}-8\end{array}$ & $\begin{array}{c}2.3880636 \mathrm{E}-4 \\
0.0002 \\
2.2 \mathrm{E}-4\end{array}$ & $\begin{array}{c}0.050475567 \\
- \\
0.8191\end{array}$ \\
\hline 128 & $\begin{array}{c}4.3630845 \mathrm{E}-22 \\
0.0000 \\
3.4 \mathrm{E}-22\end{array}$ & $\begin{array}{c}6.3877966 \mathrm{E}-16 \\
- \\
5.4 \mathrm{E}-16\end{array}$ & $\begin{array}{c}9.4418098 \mathrm{E}-8 \\
0.0000 \\
8.8 \mathrm{E}-8\end{array}$ & $\begin{array}{c}0.025786253 \\
- \\
0.6936\end{array}$ \\
\hline 256 & $\begin{array}{c}1.5978619 \mathrm{E}-43 \\
0.0000 \\
1.2 \mathrm{E}-43\end{array}$ & $\begin{array}{c}4.0803551 \mathrm{E}-31 \\
- \\
3.4 \mathrm{E}-31\end{array}$ & $\begin{array}{c}1.4759581 \mathrm{E}-14 \\
0.0000 \\
1.4 \mathrm{E}-14\end{array}$ & $\begin{array}{c}0.013033350 \\
- \\
0.5309\end{array}$ \\
\hline 512 & $\begin{array}{c}2.1430408 \mathrm{E}-86 \\
0.0000 \\
1.7 \mathrm{E}-86\end{array}$ & $\begin{array}{c}1.6649137 \mathrm{E}-61 \\
- \\
1.4 \mathrm{E}-61\end{array}$ & $\begin{array}{c}3.6067131 \mathrm{E}-28 \\
0.0000 \\
3.4 \mathrm{E}-28\end{array}$ & $\begin{array}{c}6.5494782 \mathrm{E}-3 \\
- \\
0.3614\end{array}$ \\
\hline \multicolumn{5}{|c|}{$g=0.875$} \\
\hline$\tau / \omega_{0}$ & 0.2 & 0.4 & 0.6 & 0.8 \\
\hline $2^{-15}$ & $\begin{array}{c}0.99997541 \\
- \\
0.9999764\end{array}$ & $\begin{array}{c}0.99998133 \\
- \\
0.9999807\end{array}$ & $\begin{array}{c}0.99998726 \\
1.0000 \\
0.9999860\end{array}$ & $\begin{array}{c}0.99999318 \\
- \\
0.9999928\end{array}$ \\
\hline $2^{-14}$ & $\begin{array}{c}0.99995081 \\
- \\
0.9999529\end{array}$ & $\begin{array}{c}0.99996266 \\
- \\
0.9999615\end{array}$ & $\begin{array}{c}0.99997451 \\
1.0000 \\
0.9999720\end{array}$ & $\begin{array}{c}0.99998636 \\
- \\
0.9999856\end{array}$ \\
\hline $2^{-13}$ & $\begin{array}{c}0.99990163 \\
- \\
0.9999057\end{array}$ & $\begin{array}{c}0.99992533 \\
- \\
0.9999230\end{array}$ & $\begin{array}{c}0.99994902 \\
0.9999 \\
0.9999441\end{array}$ & $\begin{array}{c}0.99997272 \\
- \\
0.9999711\end{array}$ \\
\hline $2^{-12}$ & $\begin{array}{c}0.99980327 \\
- \\
0.999811\end{array}$ & $\begin{array}{c}0.99985066 \\
- \\
0.999846\end{array}$ & $\begin{array}{c}0.99989804 \\
0.9999 \\
0.999888\end{array}$ & $\begin{array}{c}0.99994543 \\
- \\
0.999942\end{array}$ \\
\hline $2^{-11}$ & $\begin{array}{c}0.99960658 \\
- \\
0.999623\end{array}$ & $\begin{array}{c}0.99970133 \\
- \\
0.999692\end{array}$ & $\begin{array}{c}0.99979609 \\
0.9998 \\
0.999776\end{array}$ & $\begin{array}{c}0.99989087 \\
- \\
0.999884\end{array}$ \\
\hline $2^{-10}$ & $\begin{array}{c}0.99921329 \\
- \\
0.999246\end{array}$ & $\begin{array}{c}0.99940272 \\
- \\
0.999384\end{array}$ & $\begin{array}{c}0.99959220 \\
0.9996 \\
0.999553\end{array}$ & $\begin{array}{c}0.99978173 \\
- \\
0.999769\end{array}$ \\
\hline $2^{-9}$ & $\begin{array}{c}0.99842714 \\
- \\
0.99849\end{array}$ & $\begin{array}{c}0.99880570 \\
- \\
0.99877\end{array}$ & $\begin{array}{c}0.99918446 \\
0.9992 \\
0.99911\end{array}$ & $\begin{array}{c}0.99956343 \\
- \\
0.99954\end{array}$ \\
\hline $2^{-8}$ & 0.99685654 & 0.99761250 & 0.99836925 & 0.99912679 \\
\hline
\end{tabular}




\begin{tabular}{|c|c|c|c|c|}
\hline & 0.99699 & 0.99754 & 0.99821 & 0.99908 \\
\hline $2^{-7}$ & $\begin{array}{c}0.99372220 \\
- \\
0.9940\end{array}$ & $\begin{array}{c}0.99522951 \\
- \\
0.9951\end{array}$ & $\begin{array}{c}0.99673990 \\
0.9967 \\
0.9964\end{array}$ & $\begin{array}{c}0.99825340 \\
- \\
0.9982\end{array}$ \\
\hline $2^{-6}$ & $\begin{array}{c}0.98748111 \\
- \\
0.9880\end{array}$ & $\begin{array}{c}0.99047754 \\
- \\
0.9902\end{array}$ & $\begin{array}{c}0.99348589 \\
0.9935 \\
0.9929\end{array}$ & $\begin{array}{c}0.99650635 \\
- \\
0.9963\end{array}$ \\
\hline $2^{-5}$ & $\begin{array}{c}0.97510948 \\
- \\
0.9761\end{array}$ & $\begin{array}{c}0.98103067 \\
- \\
0.9805\end{array}$ & $\begin{array}{c}0.98699783 \\
0.9870 \\
0.9858\end{array}$ & $\begin{array}{c}0.99301206 \\
- \\
0.9926\end{array}$ \\
\hline $2^{-4}$ & $\begin{array}{c}0.95080631 \\
- \\
0.9528\end{array}$ & $\begin{array}{c}0.96236892 \\
- \\
0.9613\end{array}$ & $\begin{array}{c}0.97410669 \\
0.9741 \\
0.9717\end{array}$ & $\begin{array}{c}0.98602633 \\
- \\
0.9853\end{array}$ \\
\hline $2^{-3}$ & $\begin{array}{c}0.90392813 \\
- \\
0.9079\end{array}$ & $\begin{array}{c}0.92597945 \\
- \\
0.9241\end{array}$ & $\begin{array}{c}0.94868398 \\
0.9487 \\
0.9443\end{array}$ & $\begin{array}{c}0.97208165 \\
- \\
0.9707\end{array}$ \\
\hline $2^{-2}$ & $\begin{array}{c}0.8167716 \\
- \\
0.8241\end{array}$ & $\begin{array}{c}0.85689088 \\
- \\
0.8358\end{array}$ & $\begin{array}{c}0.89934479 \\
0.8993 \\
0.8914\end{array}$ & $\begin{array}{c}0.94436688 \\
- \\
0.9421\end{array}$ \\
\hline 0.5 & $\begin{array}{c}0.66628676 \\
- \\
0.6786\end{array}$ & $\begin{array}{c}0.73272216 \\
- \\
0.7285\end{array}$ & $\begin{array}{c}0.80683198 \\
0.8068 \\
0.7941\end{array}$ & $\begin{array}{c}0.88992968 \\
- \\
0.8866\end{array}$ \\
\hline 1 & $\begin{array}{c}0.44222048 \\
- \\
0.4593\end{array}$ & $\begin{array}{c}0.53331657 \\
- \\
0.5292\end{array}$ & $\begin{array}{c}0.6457714 \\
0.6458 \\
0.6287\end{array}$ & $\begin{array}{c}0.78624768 \\
- \\
0.7829\end{array}$ \\
\hline 2 & $\begin{array}{c}0.19330774 \\
- \\
0.2087\end{array}$ & $\begin{array}{c}0.27873559 \\
- \\
0.2771\end{array}$ & $\begin{array}{c}0.40672784 \\
0.4067 \\
0.3907\end{array}$ & $\begin{array}{c}0.60372727 \\
- \\
0.6035\end{array}$ \\
\hline 4 & $\begin{array}{c}0.036162388 \\
- \\
0.0418\end{array}$ & $\begin{array}{c}0.073343488 \\
- \\
0.0739\end{array}$ & $\begin{array}{c}0.15387510 \\
0.1539 \\
0.1463\end{array}$ & $\begin{array}{c}0.33942368 \\
- \\
0.3438\end{array}$ \\
\hline 8 & $\begin{array}{c}1.1993751 \mathrm{E}-3 \\
- \\
1.5 \mathrm{E}-3\end{array}$ & $\begin{array}{c}4.6333562 \mathrm{E}-3 \\
- \\
4.8 \mathrm{E}-3\end{array}$ & $\begin{array}{c}0.019672064 \\
0.0197 \\
0.0186\end{array}$ & $\begin{array}{c}0.095921527 \\
- \\
0.0977\end{array}$ \\
\hline 16 & $\begin{array}{c}1.1711948 \mathrm{E}-6 \\
- \\
1.5 \mathrm{E}-6\end{array}$ & $\begin{array}{c}1.5441339 \mathrm{E}-5 \\
- \\
1.5 \mathrm{E}-5\end{array}$ & $\begin{array}{c}2.6444028 \mathrm{E}-4 \\
0.0003 \\
2.4 \mathrm{E}-4\end{array}$ & $\begin{array}{c}6.4609227 \mathrm{E}-3 \\
- \\
5.9 \mathrm{E}-3\end{array}$ \\
\hline 32 & $\begin{array}{c}9.0503787 \mathrm{E}-13 \\
- \\
8.8 \mathrm{E}-13\end{array}$ & $\begin{array}{c}1.3633541 \mathrm{E}-10 \\
- \\
1.1 \mathrm{E}-10\end{array}$ & $\begin{array}{c}3.9983094 \mathrm{E}-8 \\
0.0000 \\
2.8 \mathrm{E}-8\end{array}$ & $\begin{array}{c}2.6204955 \mathrm{E}-5 \\
- \\
1.8 \mathrm{E}-5\end{array}$ \\
\hline
\end{tabular}


4.2514057E-25 9.3764725E-21 8.6876189E-16 4.2345203E-10

64

$2.5 \mathrm{E}-25$

5.2E-21

0.0000

4.9E-16

2.6E-10

$8.3442979 \mathrm{E}-50$

4.3667384E-41

4.0930337E-31

0.0000

3.9E-50

2.2E-41

2.2E-31

1.1054191E-19

3.1741068E-99

$9.4695066 \mathrm{E}-82$

9.0851738E-62

$6.8 \mathrm{E}-20$

256

1.4E-99

4.7E-82

0.0000

$4.9 \mathrm{E}-62$

4.6E-39

4.5924438E-198 4.4531539E-163

512

2.1E-198

2.2E-163

4.4761960E-123

3.4983517E-77

0.0000

$g=0.875$

$2.4 \mathrm{E}-123$

2.2E-77

\begin{tabular}{ccccc}
\hline$\tau / \omega 0$ & $\mathbf{0 . 9}$ & $\mathbf{0 . 9 5}$ & $\mathbf{0 . 9 9}$ & $\mathbf{1}$ \\
\hline \multirow{2}{*}{$\mathbf{2}^{-15}$} & 0.99999614 & 0.99999762 & 0.99999881 & 0.99999910 \\
& 1.0000 & - & 1.0000 & - \\
& 0.9999960 & 0.9999975 & 0.9999986 & 0.9999999 \\
$\mathbf{2}^{-14}$ & 0.99999228 & 0.99999525 & 0.99999761 & 0.99999821 \\
& 1.0000 & - & 1.0000 & - \\
& 0.9999920 & 0.9999950 & 0.9999973 & 0.9999997 \\
$\mathbf{2}^{-13}$ & 0.99998457 & 0.99999049 & 0.99999523 & 0.99999641 \\
& 1.0000 & - & 1.0000 & - \\
& 0.9999840 & 0.9999899 & 0.9999945 & 0.9999994 \\
$\mathbf{2}^{-12}$ & 0.99996913 & 0.99998098 & 0.99999046 & 0.99999283 \\
& 1.0000 & - & 1.0000 & - \\
& 0.999968 & 0.999980 & 0.999989 & 0.99999 \\
$\mathbf{2}^{-11}$ & 0.99993826 & 0.99996196 & 0.99998092 & 0.99998566 \\
& 0.9999 & - & 1.0000 & - \\
& 0.999936 & 0.999960 & 0.999978 & 0.999998 \\
$\mathbf{2}^{-10}$ & 0.99987651 & 0.99992391 & 0.99996183 & 0.99997131 \\
& 0.9999 & - & 1.0000 & - \\
& 0.999872 & 0.999919 & 0.999956 & 0.999995 \\
$\mathbf{2}^{-9}$ & 0.99975299 & 0.99984779 & 0.99992364 & 0.99994260 \\
& 0.9998 & - & 0.9999 & - \\
& 0.99974 & 0.99984 & 0.99991 & 0.99999 \\
$\mathbf{2}^{-8}$ & 0.99950586 & 0.99969548 & 0.99984720 & 0.99988514 \\
& 0.9995 & - & 0.9998 & - \\
& 0.99949 & 0.99968 & 0.99983 & 0.99998 \\
$\mathbf{2}^{-7}$ & 0.99901132 & 0.99939058 & 0.99969412 & 0.99977003 \\
& 0.9990 & - & 0.9997 & - \\
& 0.9990 & 0.9994 & 0.9997 & 0.99996 \\
& & & &
\end{tabular}




\begin{tabular}{|c|c|c|c|c|}
\hline $2^{-6}$ & $\begin{array}{c}0.99802118 \\
0.9980 \\
0.9980\end{array}$ & $\begin{array}{c}0.99877976 \\
- \\
0.9987\end{array}$ & $\begin{array}{c}0.99938718 \\
0.9994 \\
0.9993\end{array}$ & $\begin{array}{c}0.99953911 \\
- \\
0.99993\end{array}$ \\
\hline $2^{-5}$ & $\begin{array}{c}0.99603721 \\
0.9960 \\
0.9959\end{array}$ & $\begin{array}{c}0.99755437 \\
- \\
0.9974\end{array}$ & $\begin{array}{c}0.99877032 \\
0.9988 \\
0.9986\end{array}$ & $\begin{array}{c}0.99907461 \\
- \\
0.999986\end{array}$ \\
\hline $2^{-4}$ & $\begin{array}{c}0.99205677 \\
0.9921 \\
0.9918\end{array}$ & $\begin{array}{c}0.99509011 \\
- \\
0.9948\end{array}$ & $\begin{array}{c}0.99752563 \\
0.9975 \\
0.9972\end{array}$ & $\begin{array}{c}0.99813575 \\
- \\
0.9997\end{array}$ \\
\hline $2^{-3}$ & $\begin{array}{c}0.98405552 \\
0.9841 \\
0.9836\end{array}$ & $\begin{array}{c}0.99011435 \\
- \\
0.9896\end{array}$ & $\begin{array}{c}0.99499686 \\
0.9950 \\
0.9944\end{array}$ & $\begin{array}{c}0.99622248 \\
- \\
0.9994\end{array}$ \\
\hline $2^{-2}$ & $\begin{array}{c}0.96793463 \\
0.9679 \\
0.9673\end{array}$ & $\begin{array}{c}0.98000346 \\
- \\
0.9792\end{array}$ & $\begin{array}{c}0.98980188 \\
0.9898 \\
0.9886\end{array}$ & $\begin{array}{c}0.99227194 \\
- \\
0.9988\end{array}$ \\
\hline 0.5 & $\begin{array}{c}0.93541354 \\
0.9354 \\
0.9348\end{array}$ & $\begin{array}{c}0.95927562 \\
- \\
0.9580\end{array}$ & $\begin{array}{c}0.97895027 \\
0.9790 \\
0.9769\end{array}$ & $\begin{array}{c}0.98395429 \\
- \\
0.9976\end{array}$ \\
\hline 1 & $\begin{array}{c}0.87016613 \\
0.8702 \\
0.8705\end{array}$ & $\begin{array}{c}0.91640836 \\
- \\
0.9149\end{array}$ & $\begin{array}{c}0.95579846 \\
0.9558 \\
0.9525\end{array}$ & $\begin{array}{c}0.96601138 \\
- \\
0.9950\end{array}$ \\
\hline 2 & $\begin{array}{c}0.74319369 \\
0.7432 \\
0.7466\end{array}$ & $\begin{array}{c}0.82809607 \\
- \\
0.8269\end{array}$ & $\begin{array}{c}0.90566549 \\
0.9057 \\
0.9007\end{array}$ & $\begin{array}{c}0.92666165 \\
- \\
0.9891\end{array}$ \\
\hline 4 & $\begin{array}{c}0.52119310 \\
0.5212 \\
0.5272\end{array}$ & $\begin{array}{c}0.65633478 \\
- \\
0.6534\end{array}$ & $\begin{array}{c}0.80012837 \\
0.8001 \\
0.7908\end{array}$ & $\begin{array}{c}0.84310693 \\
- \\
0.9753\end{array}$ \\
\hline 8 & $\begin{array}{c}0.23345288 \\
0.2335 \\
0.2317\end{array}$ & $\begin{array}{c}0.38468650 \\
- \\
0.3701\end{array}$ & $\begin{array}{c}0.60807846 \\
0.6081 \\
0.5853\end{array}$ & $\begin{array}{c}0.69193279 \\
- \\
0.9417\end{array}$ \\
\hline 16 & $\begin{array}{c}0.04098367 \\
0.0410 \\
0.0355\end{array}$ & $\begin{array}{c}0.12049681 \\
- \\
0.1029\end{array}$ & $\begin{array}{c}0.35211631 \\
0.3521 \\
0.3194\end{array}$ & $\begin{array}{c}0.49445767 \\
- \\
0.8718\end{array}$ \\
\hline 32 & $\begin{array}{c}1.1732172 \mathrm{E}-3 \\
0.0012 \\
8.4 \mathrm{E}-4\end{array}$ & $\begin{array}{c}0.011312487 \\
- \\
8.7 E-3\end{array}$ & $\begin{array}{c}0.12625769 \\
0.1263 \\
0.1105\end{array}$ & $\begin{array}{c}0.31239611 \\
- \\
0.7629\end{array}$ \\
\hline 64 & $\begin{array}{c}9.5317157 \mathrm{E}-7 \\
0.0000 \\
6.6 \mathrm{E}-7\end{array}$ & $\begin{array}{c}9.9308855 E-5 \\
- \\
7.5 E-5\end{array}$ & $\begin{array}{c}0.017114374 \\
0.0171 \\
0.0149\end{array}$ & $\begin{array}{c}0.17986057 \\
- \\
0.6159\end{array}$ \\
\hline 128 & $\begin{array}{c}6.2910498 \mathrm{E}-13 \\
0.0000\end{array}$ & $\begin{array}{c}\text { 7.6531156E-9 } \\
-\end{array}$ & $\begin{array}{c}3.1726422 E-4 \\
0.0003\end{array}$ & $\begin{array}{c}0.097299592 \\
-\end{array}$ \\
\hline
\end{tabular}




\begin{tabular}{ccccc} 
& $4.3 \mathrm{E}-13$ & $5.8 \mathrm{E}-9$ & $2.8 \mathrm{E}-4$ & 0.4450 \\
& $2.7404835 \mathrm{E}-25$ & $4.5450522 \mathrm{E}-17$ & $1.0904721 \mathrm{E}-7$ & 0.050726846 \\
256 & 0.0000 & - & 0.0000 & - \\
& $1.9 \mathrm{E}-25$ & $3.4 \mathrm{E}-17$ & $9.5 \mathrm{E}-8$ & 0.2681 \\
& $5.2003833 \mathrm{E}-50$ & $1.6030242 \mathrm{E}-33$ & $1.2882540 \mathrm{E}-14$ & 0.025913835 \\
$\mathbf{5 1 2}$ & 0.0000 & - & 0.0000 & - \\
& $3.6 \mathrm{E}-50$ & $1.2 \mathrm{E}-33$ & $1.1 \mathrm{E}-14$ & 0.1670 \\
\hline
\end{tabular}

Our estimations of the maxima of relative errors in Prahl's (1995) $T_{\mathrm{p}}(\tau)$ values relative to IPOL's values are $-17 \%,-16 \%$, and $13 \%$ at $\tau \leq 64, \omega_{0} \leq 0.99$, and $g=0,0.5$, and 0.875 , respectively. For the same conditions, the maxima of relative errors $\delta T_{p}(\tau)$ derived from the HKS 2 model (\#4 in Table 6) are $-44 \%,-57 \%$, and $-44 \%$ (Fig. 16). Normally, errors increased with $\tau$ for the both Prahl's (1995) and HKS $2 T_{\mathrm{p}}(\tau)$ computations.
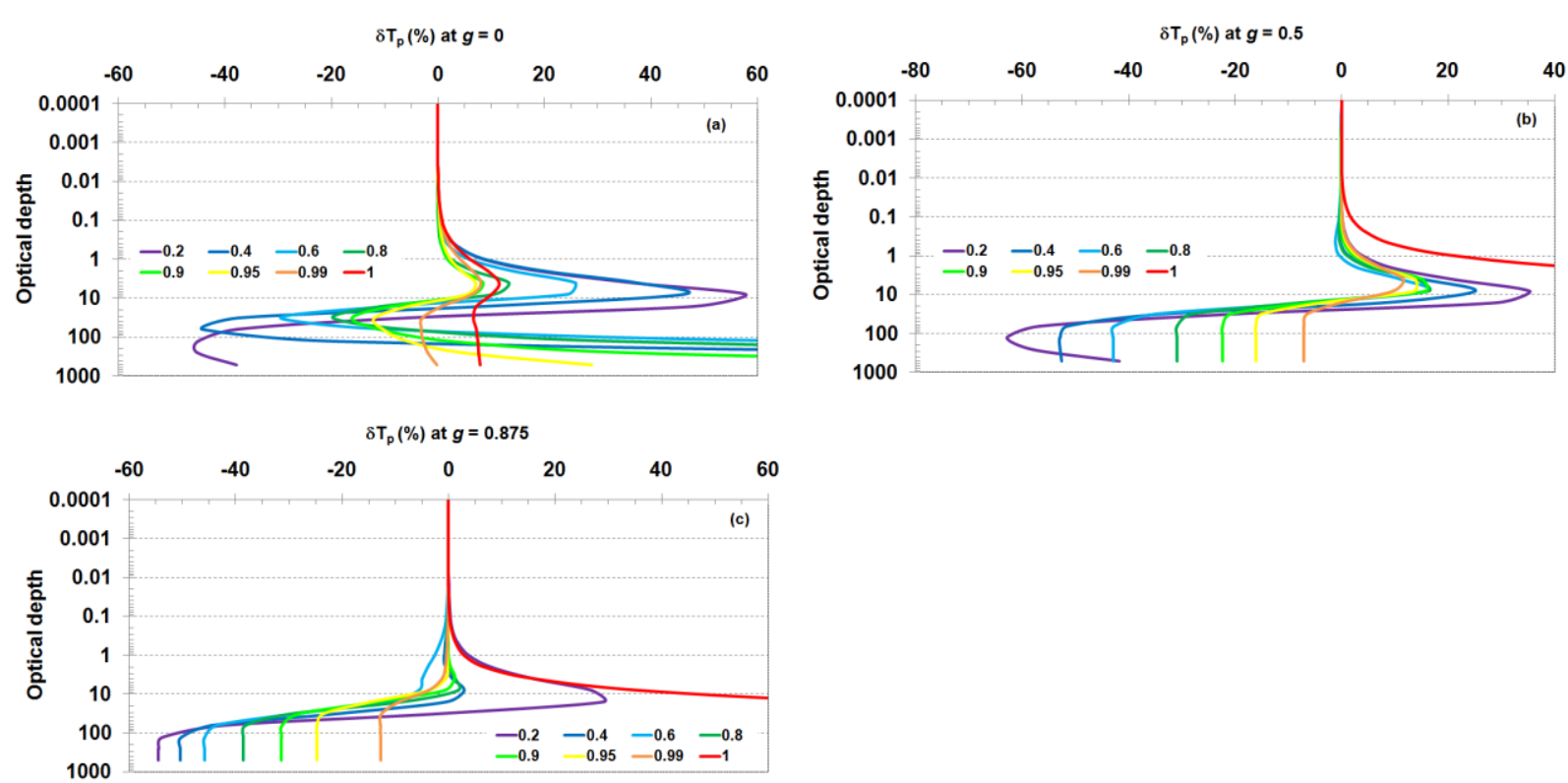

Fig. 16. The vertical profiles of relative errors in $T_{p}(\tau)$ derived by the HKS 2 model (corresponds to \#4 in Table 6) relative to IPOL-based values for the Henyey-Greenstein 
$p(\theta)$ at three values of $g: 0(a), 0.5(b)$, and $0.875(\mathrm{c}) ; \theta_{i}=0^{\circ}$; and selected values of $\omega_{0}$ (shown in the legends).

However, after conversion of $T_{\mathrm{p}}(\tau)$ to $\bar{K}_{\mathrm{d} \text {,dir }}(0-\rightarrow \tau) / c$ values, a character of errors $\delta \bar{K}_{\mathrm{d}, \text { dir }}(0-\rightarrow \tau) / c$ becomes different from the $\delta T_{\mathrm{p}}(\tau): \delta \bar{K}_{\mathrm{d}, \mathrm{dir}}(0-\rightarrow \tau) / c$ generally decreases with the optical depth for the both Prahl's (1995) and HKS 2 methods. For example, $\delta \bar{K}_{\mathrm{d} \text {,dir }}(0-\rightarrow \tau) / c$ for Prahl's (1995) method is $105 \%, 63 \%$, and $96 \%$ at $\tau \leq 2^{-13}$, $\omega_{0} \leq 0.99$, and $g=0,0.5$, and 0.875 , respectively; however, errors do not exceed $2.4 \%$ at $\tau \geq 2^{-5}$. The maximal values of errors $\delta \bar{K}_{\mathrm{d} \text {,ir }}(0-\rightarrow \tau) / c$ found for the HKS 2 model (\#4 in Table 6) are $-12 \%, 12 \%$, and $15 \%$ at $\tau \leq 2^{-13}, \omega_{0} \leq 0.99$, and $g=0,0.5$, and 0.875 , respectively (Fig. 17). At $\tau>2^{-13}$, errors strongly decreased at $g=0$ and $g=0.875$; however, at $g=0.5$, HKS 2 -induced errors achieve a maximum of $-17 \%$ at $\tau=2$ and $\omega_{0}$ $=0.99$; after that, errors sharply decreased (Fig. 17). Note that at $\omega_{0}=1$ combined with $g=0.5$ or 0.875 , errors in HKS 2 are very large (from $-51 \%$ to $-99 \%$ ) and not shown here. All these results are in agreement with the results obtained for normal light incidence on a layer characterizing by the four other scattering phase functions (Fig. 13). 

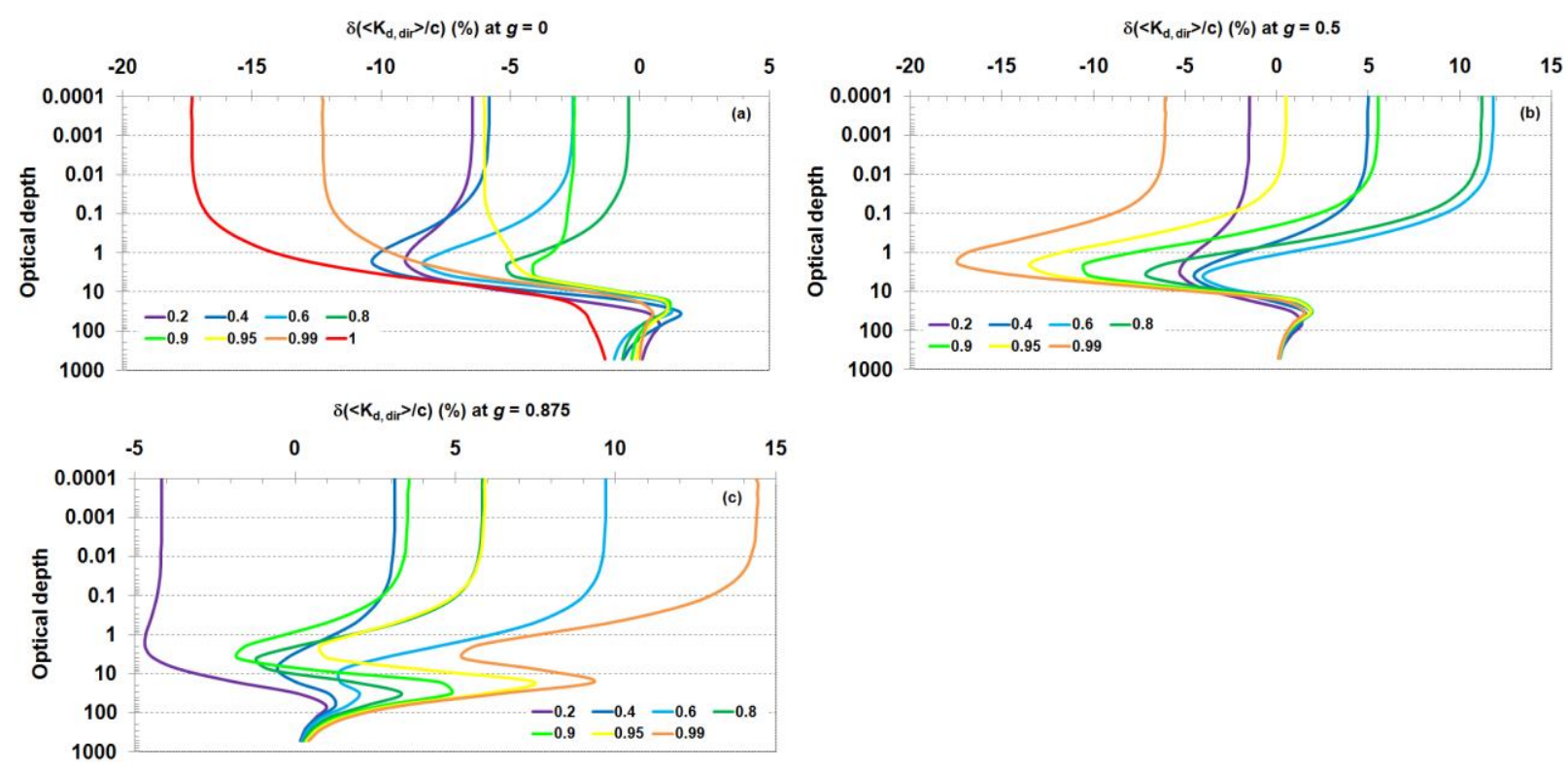

Fig. 17. The same as Fig. 16 , but for $\bar{K}_{\mathrm{d}, \mathrm{dir}}(0-\rightarrow \tau) / c$.

\subsection{Combined (direct and diffuse) illumination}

It is easy to derive equations for combined (direct and diffuse) illumination. The transmittance through the optical depth (thickness) under combined illumination, $T_{\mathrm{c}}(\tau)$ :

$$
T_{\mathrm{c}}(\tau)=\frac{E_{\mathrm{d}}(\tau)}{E_{\mathrm{d}}(0-)}=\frac{E_{\mathrm{d}, \mathrm{dir}}(0-) T_{\mathrm{p}}(\tau)+E_{\mathrm{d}, \mathrm{dif}}(0-) t(\tau)}{E_{\mathrm{d}}(0-)}=\left[1-d_{\mathrm{E}}(0-)\right] T_{\mathrm{p}}(\tau)+d_{\mathrm{E}}(0-) t(\tau)
$$

where $d_{E}(0-)$ is the diffuseness of irradiance, defined as a contribution of diffuse irradiance $E_{\mathrm{d} \text {, dif }}(0-)$ to the total irradiance, $E_{\mathrm{d}}(0-)$ just after refraction on the boundary between two media. This parameter is related to the irradiance diffuseness just before refraction, $d_{E}(0+)$ :

$$
d_{\mathrm{E}}(0-)=\frac{E_{\mathrm{d}, \mathrm{dif}}(0-)}{E_{\mathrm{d}}(0-)}=\frac{E_{\mathrm{d}, \mathrm{dif}}(0+)[1-r(0+)]}{E_{\mathrm{d}}(0+)\left[1-R_{\mathrm{c}}(0+)\right]}=\frac{1-r(0+)}{1-R_{\mathrm{c}}(0+)} d_{\mathrm{E}}(0+),
$$


where $r\left(0_{+}\right)$is a spherical albedo just before refraction, which may be expressed via the relative index of refraction $n$ (see, e.g., Eq. 6 by Molenaar et al., 1999); $R_{c}\left(0_{+}\right)$is a reflectance from the upper boundary under combined illumination:

$$
R_{\mathrm{c}}(0+)=\left[1-d_{\mathrm{E}}(0+)\right] R_{\mathrm{p}}(0+)+d_{\mathrm{E}}(0+) r(+),
$$

where $R_{p}\left(0_{+}\right)$is a plane albedo just before refraction, which may be calculated via incident angle before refraction $\theta_{0}$ and refraction index $n$ by the classical Fresnel's law (see, e.g., Eq. 4 by Molenaar et al., 1999).

In the case an outer layer is the Earth's atmosphere, parameter $d_{E}(0+)$ may be determined as a function of wavelength, solar zenith angle, and aerosol optical thickness (Højerslev, 2001; Sokoletsky et al., 2014b).

Now the average attenuation coefficient for downwelling irradiance under combined illumination, $\bar{K}_{\mathrm{dc}}(0-\rightarrow \tau)$ may be easily found as

$$
\bar{K}_{\mathrm{dc}}(0-\rightarrow \tau)=-\frac{c}{\tau} \ln T_{c}(\tau)
$$

\section{Discussion and conclusions}

The main aim of the study was to reveal the analytical approximations appropriate for estimation of transmitted light in the plane-parallel layer. To this end, we considered dozens of different models for direct and diffuse illumination. Analysis has shown that, generally, analytical models yield better results in the case of diffuse illumination. 19 $\bar{K}_{\mathrm{d} \text {, dif }}(0-\rightarrow \tau)$ models have shown acceptable results under maximal level of NRMSE varied from 14\% (models \#2-7, 9-13, 19-22, 33, and 34 in Table 4) to 21\% (models 17 and 18 in the same table) at all parameters values. The $\bar{K}_{\mathrm{d} \text {, dif }}(0-\rightarrow \tau)$ models based 
on Hulst's model (models \# 6 and 7) for $r_{\infty}$ and two different algorithms for the diffusion exponent $k$, yielded the best result with a total NRMSE of $7.8 \%$.

The situation appears more complicated for direct illumination at incident angles $\theta_{i}$ beginning approximately from $60^{\circ}$ and at high single-scattering albedo $\omega_{0}$ close to 1. The two best $\bar{K}_{\mathrm{d} \text {, dir }}(0-\rightarrow \tau)$ models based on the HKS 2 approximation for the plane albedo (Kokhanovsky and Sokoletsky, 2006b; Sokoletsky et al., 2013) give relative errors almost always within $\pm 20 \%$ at any scattering phase function $p(\theta)$, optical depth (thickness) $0.001 \leq \tau \leq 10000, \theta_{i} \leq 60^{\circ}$, and $\omega_{0} \leq 0.95$ (Figs. 13-15). A total NRMSE for the same models is approximately $4 \%, 6 \%$, and $12 \%$ for $\theta_{i}$ of $0^{\circ}, 30^{\circ}$, and $60^{\circ}$, respectively.

This range of parameters certainly covers the majority of possible situations in field or laboratory observations. For example, remote-sensing observations are generally limited by $70^{\circ}-75^{\circ}$ (Martins et al., 2002; Liu et al., 2009; Lee et al., 2017). Moreover, the atmospheric plane-parallel radiative transfer calculations are limited by nadir measurements with solar zenith angles up to $75^{\circ}$ due to the sphericity of the terrestrial atmosphere (Doicu et al., 2010; Rozanov et al., 2014). Thus, a maximal incident angle after refraction on the air-water boundary may be estimated as $\theta_{i}=\arcsin (75 \% 1.340) \approx$ $46^{\circ}$ at the middle of the visible spectrum. Concerning optical instruments in laboratories, they generally use rectangular cuvettes, light on which is incident normally or nearnormally, i.e., $\theta_{\mathrm{i}} \approx 0^{\circ}$.

Observations and calculations of the other optical parameter, $\omega_{0}$, give its ranges of 0.78 to 0.99 for aerosols (Dubovik et al., 2002), 0.01 to 0.98 for natural waters (Lee, 2006; Sokoletsky and Shen, 2014), and 0.50 to 0.94 for normal and cancerous human 
skin (Salomatina et al., 2006). Similar conclusions may be found concerning $g$ and $B$ parameters.

An important conclusion of this study is that the traditional direct models for the transmittance or diffuse attenuation coefficient (such as BLB, GKM, Gordon's, Lee et al. etc.) generally perform worse (for both direct and diffuse illumination) than the models based on combined use of additional optical parameters such as the diffusion exponent $k$ (Appendix $A$ ), the average cosine $\bar{\mu}_{d}$ of the layer's light field for the downwelling irradiance (Appendix B), and albedo of infinite or semi-infinite layer $R_{\infty}$ (Appendices C and D). Modeling these optical properties is an important independent task: different approaches for each of these parameters were analyzed and reliable solutions found.

It is also worth to mentioning a difficulty of interpretation $\bar{\mu}_{\mathrm{d}}$ in the frame of the twoflux approximation GKM (Eq. B.1), which may lead to non-physical values, as shown in Appendix B. As demonstrate Eqs. (B.1)-(B.5) and Fig. B.1, $\bar{\mu}_{d}(\tau)$ in our models may reach the value much more than 1 (instead of physically correct value of just one) while $\bar{\mu}_{\mathrm{d}}(\tau=\infty)$ is always equal 1 [instead of variations in the range from 0.5 to 1 depending on $\omega_{0}$ and $p(\theta)$ ]. Nevertheless, Eqs. (B.1)-(B.5) lead to physically correct and numerically accurate solutions for transmittance and diffuse attenuation coefficient that has been confirmed by analysis of sensitivity and approximations' accuracy performed in our study. Similar conclusions may be obtained for the vertically variable reflectances (albedos), which, however, were not within the scope of this study.

In order to achieve more generality of our conclusions, we have considered two different sets of scattering phase function $p(\theta)$ : four $p(\theta)$ given by the finite FourierLegendre series (Eq. 10, Table 2, Fig. 3) and the Henyey-Greenstein $p(\theta)$ with three 
values of $g$ taken from Prahl (1995). We provided numerical values (both for IPOL and HKS 2) of plane transmittance for the Henyey-Greenstein $p(\theta)$ that allows to use them as benchmark values for the other models.

Finally, we note that the successful modeling of reflective and transmitted properties for direct and diffuse illumination opens the door to the solution of optical problems under combined (arbitrary) illumination. We have considered this issue briefly in subsection 4.3 .

\section{Appendix A. Modeling the diffusion exponent $k$}

Diffusion exponent $k$ has been calculated by four different analytical methods as follows:

(1) Kattawar and Plass (1976) approximation

$$
\begin{gathered}
A=k^{-1} \ln [(1+k) /(1-k)], \quad \alpha=6+\left(k^{2}-3\right) A, \quad \beta=2\left(k^{2}-3\right)+\left(3 k^{4}-2 k^{2}+3\right) A, \\
\gamma=-(16 / 3) k^{4}, \quad \omega_{0}=\left[-\beta+\left(\beta^{2}-4 \alpha \gamma\right)^{1 / 2}\right] /(2 \alpha),
\end{gathered}
$$

which was used for the quasi-Raleigh $p(\theta) \# 1$ (i.e., at $g=0$ ) only;

(2) Sobolev (1975) (his Eq. 22) approximation where the diffusion exponent is derived from continuing fraction (see also Nakajima and King, 1992):

$$
1-\omega_{0}=\frac{k^{2}}{3\left(1-\omega_{0} g\right)-\frac{4 k^{2}}{5\left(1-\omega_{0} g^{2}\right)-\frac{9 k^{2}}{7\left(1-\omega_{0} g^{3}\right)-\frac{16 k^{2}}{9\left(1-\omega_{0} g^{4}\right)-\ldots}}}} .
$$

(3) King and Harshvardhan (1986) model derived for the Henyey-Greenstein $p(\theta)$ (Henyey and Greenstein, 1941) as follows: 


$$
k=\left(1-\omega_{0} g\right)\left[s \sqrt{3}-\frac{(0.985-0.253 s) s^{2}}{6.464-5.464 s}\right],
$$

where $s$ is the Hulst's similarity parameter defined as (Hulst, 1974, 1980)

$$
s=\sqrt{\frac{1-\omega_{0}}{1-\omega_{0} g}} .
$$

(4) Sokoletsky et al. (2013) model, derived from Eq. (15), in which the ratios of K/c and S/c are derived from Eqs. (C.11) below as

$$
\frac{K}{c}=\frac{1-\omega_{0}}{\eta}, \frac{S}{c}=\frac{\omega_{0}(1-g)}{\chi},
$$

where variables $\eta$ and $\chi$ are expressed through the transport single-scattering albedo according to Eqs. (C.20) and (C.21).

All four $k$ models were compared with the numerical results obtained by Hulst (1980) for isotropic scattering $(g=0)$, and by Dlugach \& Yanovitskij (1974) for any $g$ (Fig. A.1); errors of different analytical models shown in Figs. A.2 and A.3.

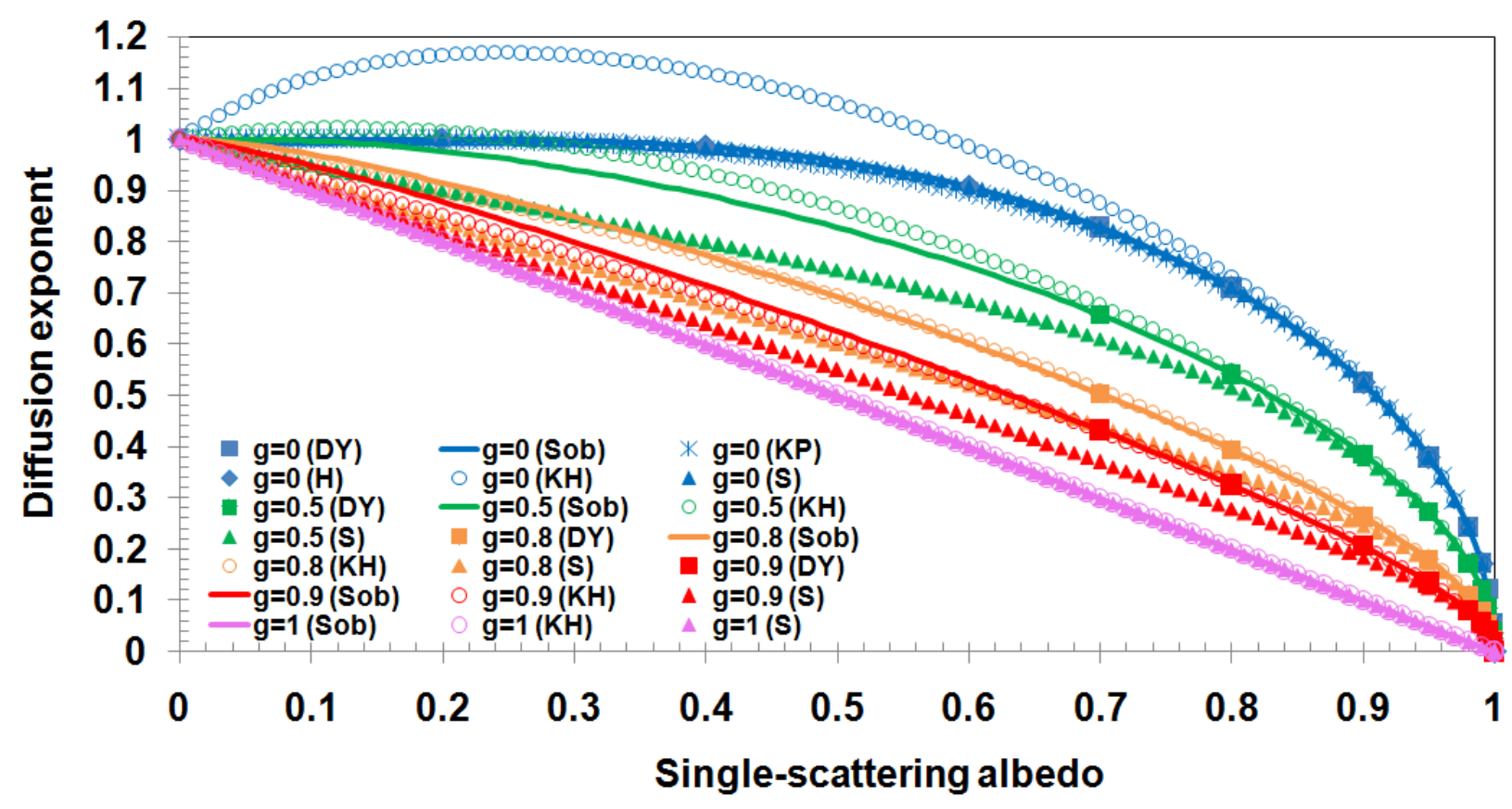


Fig. A.1. The diffusion exponent $k$ as a function of $\omega_{0}$ at selected values of $g$ plotted according to numerical computation by Dlugach \& Yanovitskij (1974) (abbreviated as "DY" and shown by squares) and Hulst (1980) ("H", rhombs), and analytical models by Sobolev (1975) ("Sob", solid curves), Kattawar and Plass (1976) ("KP", asterisks), King and Harshvardhan (1986) ("KH", circles), and Sokoletsky et al. (2013) ("S", triangles).
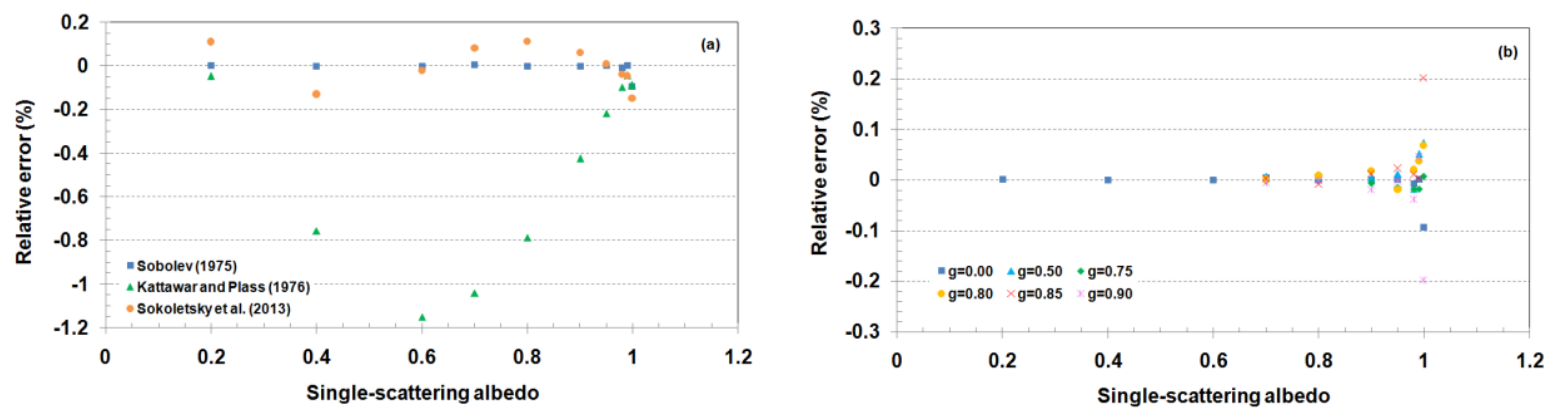

Fig. A.2. Relative errors for the diffusion exponent calculated by Sobolev's (1975) continuing fraction [Eq. (A.3) taken with 140 terms] (a, b) and by analytical approaches by Kattawar and Plass (1976) and Sokoletsky et al. (2013) for the case of $g=0$ (a) relative to the numerical computations performed by Dlugach \& Yanovitskij (1974) and Hulst (1980) for the Henyey-Greenstein $p(\theta)$.
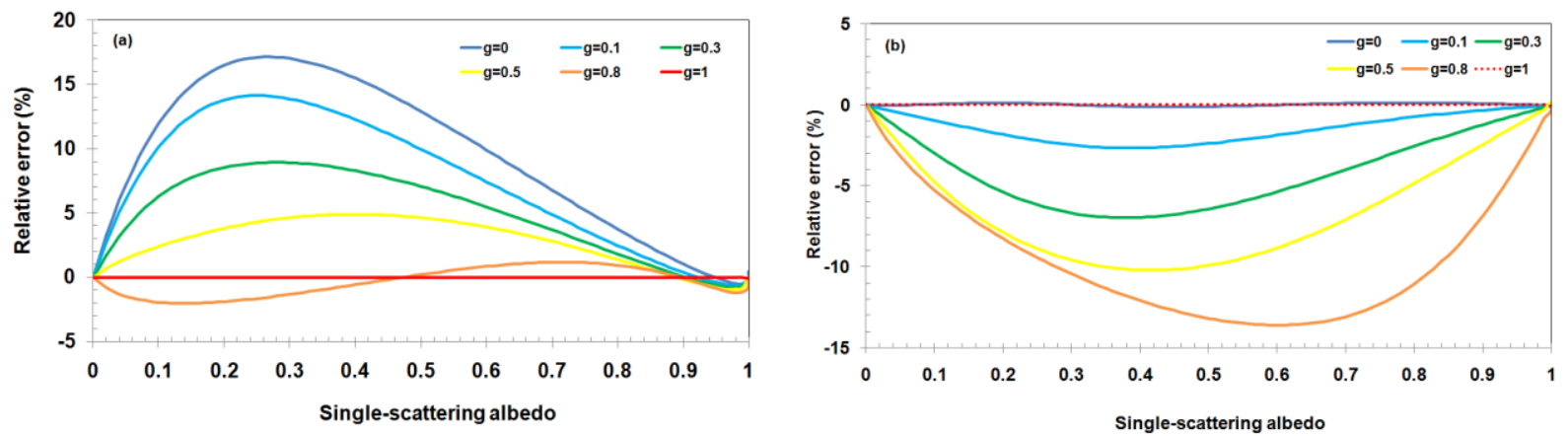

Fig. A.3. Relative errors calculated for the diffusion exponent $k$ calculated by King and Harshvardhan (1986) (a) and Sokoletsky et al. (2013) (b) relative to calculations carried 
out by Eqs. (A.1) and (A.2) (at $g=0$ ) and (A.3) (at $g>0$ ) for all $0 \leq \omega_{0} \leq 1$ at selected $g$ indicated in the legend.

Fig. A. 1 shows that $k=1$ at $\omega_{0}=0$ and $k=0$ at $\omega_{0}=1$ independent of $p(\theta)$ or $g$. Moreover, according to all models excluding the King and Harshvardhan (1986) model, the diffusion exponent is a strictly decreasing (concave downward) function of $\omega_{0}$ at all $g$ excluding $g=1$ when all models give a strictly linear function $k=1-\omega_{0}$. The last is reasonable because the case of $g=1$ means total forward scattering at which there is no reflected light $\left[r(\tau)=R_{\mathrm{p}}(\tau)=0\right]$ and transmitted light depends only on absorption. Mathematically, it is equivalent to the generalized BLB law:

$$
\left.T(\tau)\right|_{g=1}=\exp \left(-k \tau_{\mathrm{ef}}\right)=\exp \left[-\left(1-\omega_{0}\right) \tau_{\mathrm{ef}}\right]=\exp \left(-a z / \bar{\mu}_{\mathrm{d}}\right)
$$

as it follows from Eq. (16) at $R_{\infty}=0$ and $k=1-\omega 0$.

The main model for the diffusion exponent $k$ used for this study, namely, Sobolev's (1975) (Eq. A.3 above) model, has shown excellent results with the relative errors less than $0.2 \%$ for $p(\theta)$ with $0 \leq g \leq 0.9$ at $0 \leq \omega_{0} \leq 1$ (Fig. A.2). However, Sobolev's (1975) method has three disadvantages: 1) this is not a direct method, generally demanding a large number of the terms of continuing fraction (Eq. A.3); 2) the solutions for $k$ are not unique, which necessitates preliminary establishment of the ranges for $k$ at each set of $g$ and $\omega_{0}$; and 3 ) at $\omega_{0}$ close to $0, k$ may be slightly more than 1 (Fig. A.4), which is physically incorrect. Therefore, a model by Kattawar and Plass (1976), Eqs. (A.1) and (A.2), which always yields $k \leq 1$ (Fig. A.4), has been used for the case of $p(\theta) \# 1$. 


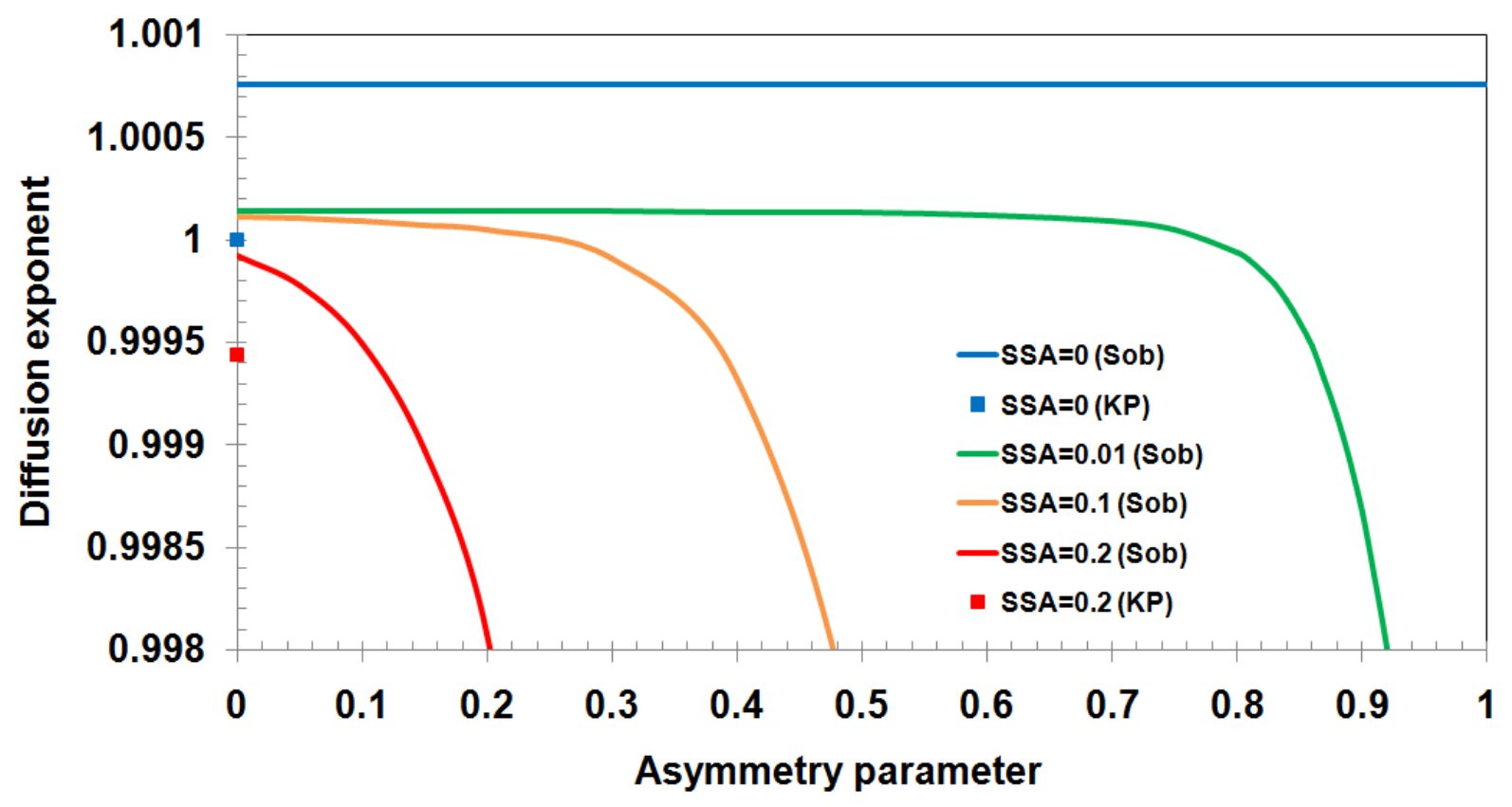

Fig. A.4. Diffusion exponent calculated by Sobolev's (1975) method (abbreviated as "Sob" and shown by solid curves) for all $0 \leq g \leq 1$ and by Kattawar and Plass (1976) model ("KP", squares) for $g=0$ at selected single-scattering albedo (SSA) indicated in the legend.

An analysis of accuracy of two analytical models yielding a direct solution for $k$, namely the King and Harshvardhan (1986) and Sokoletsky et al. (2013) models, shows that the first model differs from the numerical results at $g=0$ by up to $1.15 \%$ while the second model does so by no more than $0.15 \%$ (Fig. A.2a). Overall, at all values of $g$, the King and Harshvardhan (1986) model has an accuracy better than $9 \%$ at $g \geq 0.3$ (Fig. A.3a), which is the best result among all models tested (not shown here), and the Sokoletsky et al. (2013) model is superior at $g \leq 0.3$ (Fig. A.3b) with a relative error less than 7\%. Thus, the Kattawar and Plass (1976) and Sokoletsky et al. (2013) models 
were ultimately used for the quasi-Raleigh $p(\theta) \# 1$, while the Sobolev (1975) and King and Harshvardhan (1986) models were applied for the other phase functions.

\section{Appendix B. Modeling the average cosine for the downwelling irradiance $\bar{\mu}_{d}(\tau)$}

Eq. (16) can be easily resolved regarding $\bar{\mu}_{\mathrm{d}}(\tau)$ as

$$
\bar{\mu}_{\mathrm{d}}(\tau)=\frac{k \tau}{\ln \left\{\frac{2 T(\tau) R_{\infty}^{2}}{\sqrt{\left(1-R_{\infty}^{2}\right)^{2}+\left[2 T(\tau) R_{\infty}\right]^{2}}-\left(1-R_{\infty}^{2}\right)}\right\}} .
$$

Eq. (B.1) will be exploited in the current research for establishment of benchmark values of $\bar{\mu}_{\mathrm{d}}(\tau)$ using the $R_{\infty}$ and $T(\tau)$ values derived from the numerical solution (IPOL) of the radiative transfer equation. Further, the values of $\bar{\mu}_{d}(\tau)$ derived from Eq. (B.1) were used for development of an analytical model of $\bar{\mu}_{\mathrm{d}}(\tau)$ in the form similar to that was successfully used by Berwald et al. (1995), Sokoletsky et al. (2003), and Sokoletsky and Budak (2016b, 2017) :

$$
\bar{\mu}_{\mathrm{d}}(\tau)=\bar{\mu}_{\mathrm{d}, \infty}+\left(\bar{\mu}_{\mathrm{d}, 0}-\bar{\mu}_{\mathrm{d}, \infty}\right) \exp (-P \tau)
$$

where $\bar{\mu}_{\mathrm{d}, 0}$ and $\bar{\mu}_{\mathrm{d}, \infty}$ are the average cosine for the downwelling irradiance at $\tau=0$ - and $\tau=\infty$, respectively; $P$ is the rate of change of $\bar{\mu}_{\mathrm{d}}(\tau)$ with $\tau$. An algorithm was created for two different cases: 1) $\bar{\mu}_{\mathrm{d}}(\tau)$ for the downwelling direct irradiance $\left[\bar{\mu}_{\mathrm{d} \text {, dir }}(\tau)\right]$ and 2) $\bar{\mu}_{\mathrm{d}}(\tau)$ for the downwelling diffuse irradiance $\left[\bar{\mu}_{\mathrm{d} \text {, dif }}(\tau)\right]$ as follows:

$$
\bar{\mu}_{\mathrm{d}, \mathrm{dir}, 0}=\left(1+\sum_{\mathrm{i}=1}^{2} a_{1 \mathrm{i}}\left(\omega_{0}\right)^{\mathrm{i}}+g \sum_{\mathrm{j}=1}^{3} a_{2 \mathrm{j}}\left(\omega_{0}\right)^{\mathrm{j}}+\omega_{0} \sum_{\mathrm{k}=2}^{3} a_{3 \mathrm{k}} g^{\mathrm{k}}\right) \mu_{\mathrm{i}}
$$




$$
\begin{gathered}
\bar{\mu}_{\mathrm{d}, \mathrm{dif}, 0}=0.5+\sum_{\mathrm{i}=1}^{5} b_{1 \mathrm{i}}\left(\omega_{0}\right)^{\mathrm{i}}+g \sum_{\mathrm{j}=1}^{3} b_{2 \mathrm{j}}\left(\omega_{0}\right)^{\mathrm{j}}+\omega_{0} \sum_{\mathrm{k}=2}^{3} b_{3 \mathrm{k}} g^{\mathrm{k}}, \\
P_{\mathrm{dir}}=\sum_{\mathrm{i}=0}^{2} \alpha_{\mathrm{i}} s^{\mathrm{i}}, P_{\mathrm{dif}}=\sum_{\mathrm{i}=0}^{2} \beta_{\mathrm{i}} s^{\mathrm{i}}, \bar{\mu}_{\mathrm{d}, \mathrm{dir}, \infty}=\bar{\mu}_{\mathrm{d}, \mathrm{dif}, \infty}=1,
\end{gathered}
$$

where $s$ is determined by Eq. (A.5) and numerical coefficients are given in Table B.1.

Table B.1. Coefficients of Eqs. (B.3)-(B.5).

\begin{tabular}{ccccccc}
\hline Coef./Indices & $\mathbf{0}$ & $\mathbf{1}$ & $\mathbf{2}$ & $\mathbf{3}$ & $\mathbf{4}$ & $\mathbf{5}$ \\
\hline $\boldsymbol{a}_{1 \mathbf{i}}$ & & 1.110 & -0.7742 & & & \\
$\boldsymbol{a}_{2 \mathrm{j}}$ & & 0.3096 & -3.684 & 4.234 & & \\
$\boldsymbol{a}_{3 \mathbf{k}}$ & & & 1.111 & -1.233 & & \\
$\boldsymbol{b}_{\mathbf{1 i}}$ & & 0.2771 & 1.861 & -8.063 & 11.58 & -5.415 \\
$\boldsymbol{b}_{\mathbf{2} \mathrm{j}}$ & & -0.7394 & 1.178 & -1.013 & & \\
$\boldsymbol{b}_{\mathbf{3 k}}$ & & & 1.017 & -0.8172 & & \\
$\boldsymbol{\alpha}_{\mathrm{i}}$ & 0.1755 & 0.2126 & -0.3372 & & & \\
$\boldsymbol{\beta}_{\mathbf{i}}$ & 0.7265 & 0.4389 & -1.001 & & & \\
\hline
\end{tabular}

The model was developed so that to provide $\bar{\mu}_{\mathrm{d}, \mathrm{dir}, 0}=\mu_{\mathrm{i}}$ and $\bar{\mu}_{\mathrm{d}, \mathrm{dif}, 0}=0.5$ at $\omega_{0}=0$ (Kubelka, 1948; Judd, 1975). This means that, in the absence of scattering, a light field is completely defined by the incident illumination. However, a scattering process (determined by $\omega_{0}$ ) does change the $\bar{\mu}_{\mathrm{d}}(\tau)$ already at $z=0$-, and further at increasing depths regardless of the optical conditions of the medium. 
It is important to stress a complicate behavior of $\bar{\mu}_{\mathrm{d}}(\tau)$ within the whole range of optical depths: it may increase, decrease or be relatively stable with $\tau$ (Fig. B.1). Actually, its behavior is governed by IOPs and - especially - by illumination's conditions, namely, at small values of $\theta_{i}\left(\right.$ appr. up to $\left.30^{\circ}\right), \bar{\mu}_{d}(\tau)$ is generally decreases with the $\tau$ (see also Gordon, 1989; Bissett et al., 1999; Kirk, 2011); however, at $\theta_{\mathrm{i}}>30^{\circ}$ and at diffuse illumination, it generally increases. Such behavior is continues up to achievement of the asymptotic light regime at large values of $\tau$ (Fig. B.1).
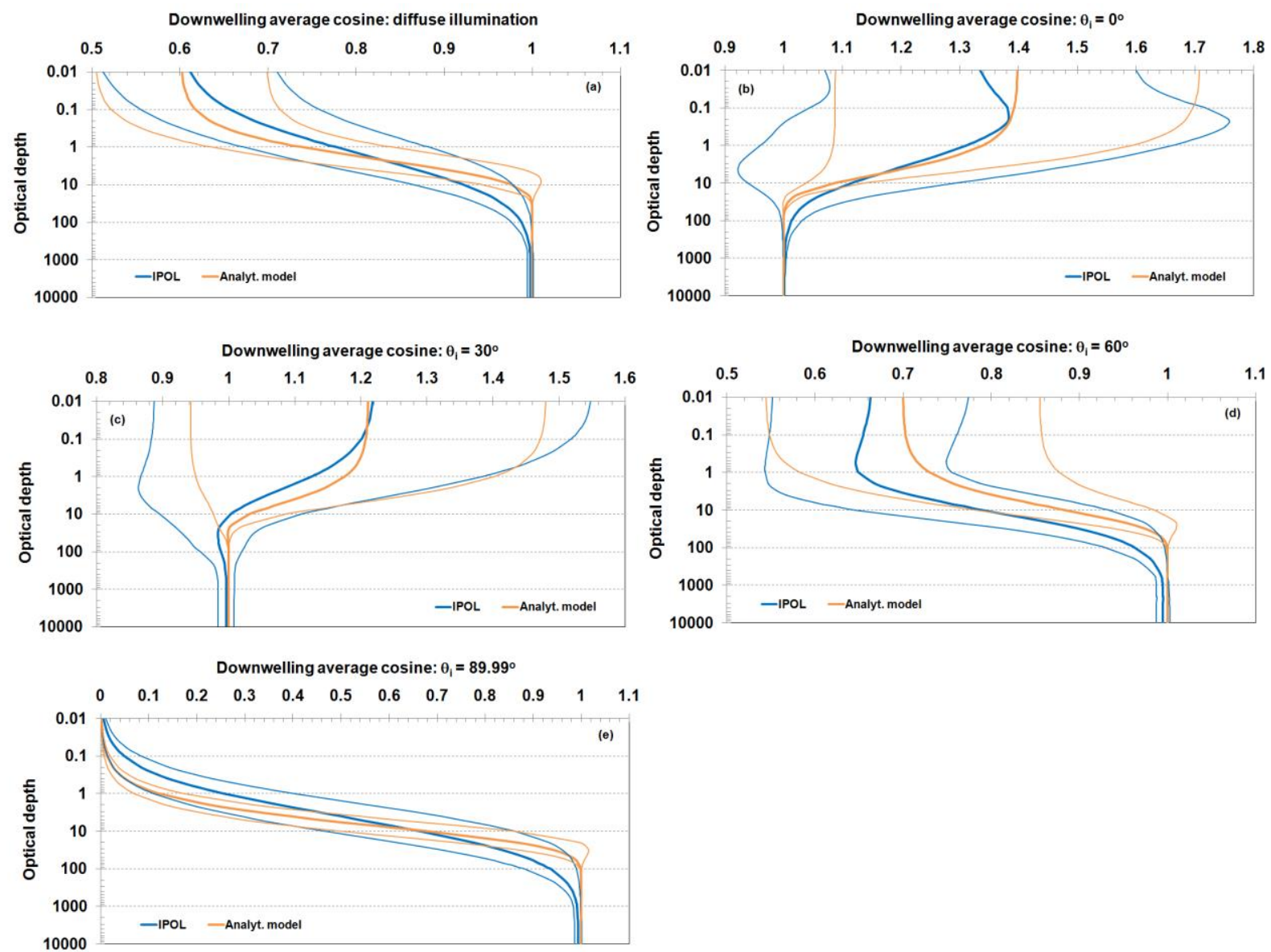

Fig. B.1. A comparison of the vertical profiles of $\bar{\mu}_{\mathrm{d} \text {, dif }}(\tau)$ and $\bar{\mu}_{\mathrm{d} \text {,dir }}(\tau)$ calculated from the IPOL's values of $R(\tau)$ and $T(\tau)$ by Eq. (B.1) (blue curves) with the corresponding optical properties calculated by the analytical equations (B.2)-(B.5) (orange curves) for: 
(a) diffuse illumination; (b) $\theta_{i}=0^{\circ}$; (c) $\theta_{i}=30^{\circ}$; (d) $\theta_{i}=60^{\circ}$; and (e) $\theta_{i}=89.99^{\circ}$. All curves are shown as the means for all selected $\omega_{0}$ and $p(\theta), \# 1-3$ from Table 2 . The thick and thin curves correspond to the mean and mean \pm standard deviation values, respectively.

However, at very large optical depths (let's say, $\tau>100$ ), photons have a tendency completely "to forget" their initial direction of motion and they become prevailingly scattered normally relative to the slab's surface in the forward (downward) part of the asymptotic light field, as expressed simply by the equality of $\bar{\mu}_{d, \infty}=1$ (Fig. B.1). Note that this statement may be derived rigorously from Eq. (B.1) after obvious replacement of $k \tau$ by $\bar{K}_{\mathrm{d}}(0-\rightarrow \infty) \tau / c=-\ln T(\infty)$ and using the equality of $T(\infty)=0$. However, this statement is somewhat in contradiction to the findings of Mobley (1994) (his Fig. 9.4), Haltrin (1998) (his Fig. 1), and Kirk (2011) (his Figs. 6.14 and 6.16), according to which $\bar{\mu}_{\mathrm{d}, \infty}=1$ only at $\mu_{\mathrm{i}}=1$ and $\omega_{0}=0$, but decreases (approximately up to 0.5 ) with increasing $\omega_{0}$ up to 1.

Therefore, using Eq. (B.1) derived from the two-flux approximation to an exact solution of the radiative transfer equation, may either give or not give the accurate values for $\bar{\mu}_{\mathrm{d}}(\tau)$ even at absolutely exact $R_{\infty}$ and $T(\tau)$ values. Thus, strictly speaking, an optical property $\bar{\mu}_{\mathrm{d}}$ in this study is considered only as an intermediate quantity or a quasi-downwelling average cosine suitable for transmittance modeling only. In addition, note the obvious differences (both quantitative and qualitative) between results derived for $\bar{\mu}_{\mathrm{d}}(\tau)$ by applying numerical and analytical methods. A reason for these differences originates from the fact that the numerically-based calculations better account for a thin 
structure of the scattering phase functions than do analytical equations (B.2-B.6) exploiting only integral optical parameters $\left(\tau, g\right.$, and $\left.\omega_{0}\right)$. Nevertheless, numerical differences between these two approaches are generally less than $10 \%$ at angles $\theta_{i}<$ $45^{\circ}$ (Fig. B.2), i.e., at most typical angles with which researchers generally come across.

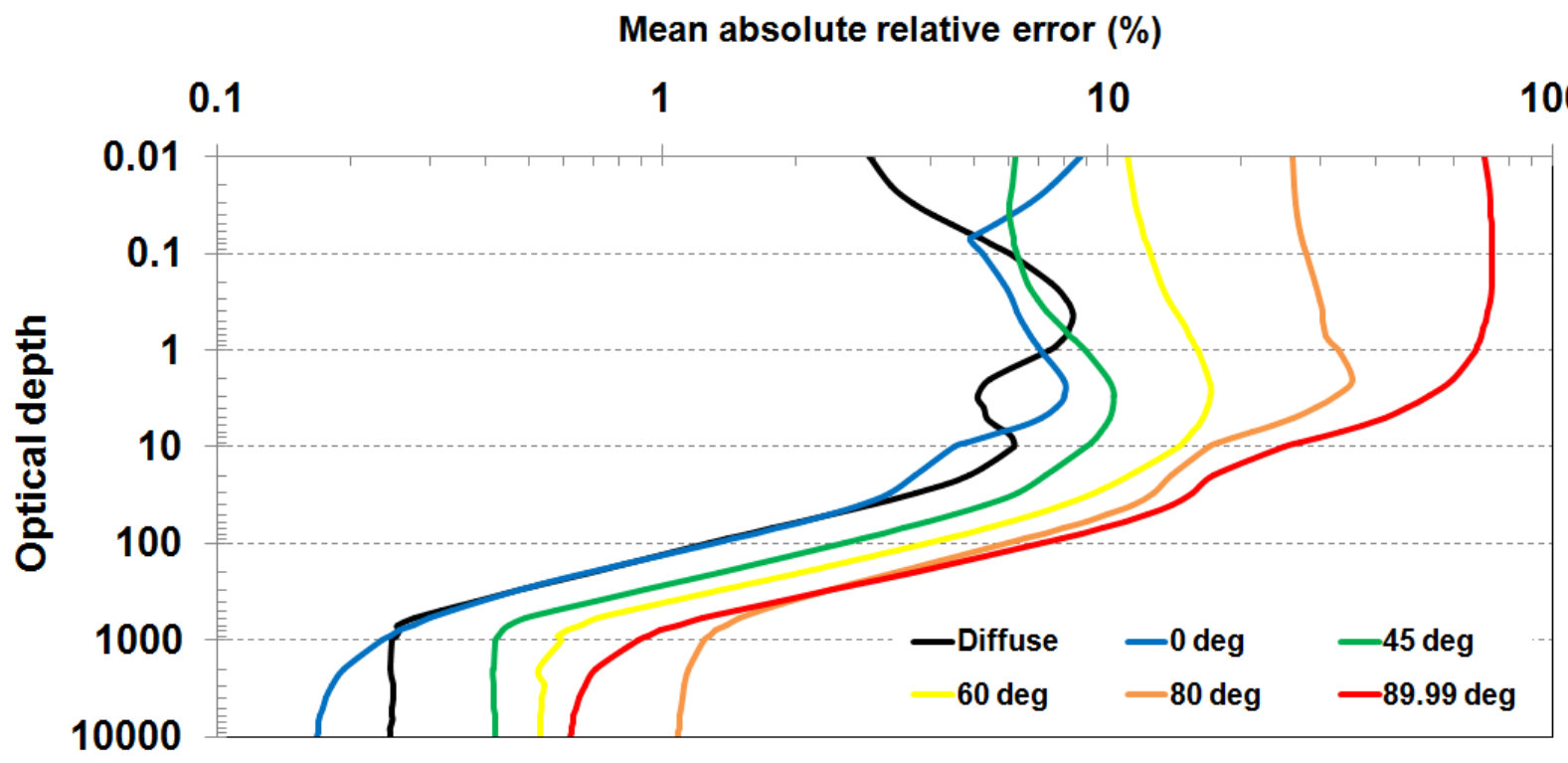

Fig. B.2. A mean absolute relative error of $\bar{\mu}_{d}(\tau)$ calculated by Eqs. (B.2)-(B.5) relative to $\bar{\mu}_{d}(\tau)$ calculated by Eq. (B.1) at selected illumination conditions (in the legends), $p(\theta) \# 1$ to 3 from Table 2, and averaged for 14 selected $\omega_{0}$.

\section{Appendix C. Modeling the spherical albedo of infinite or semi-infinite layer}

For the spherical albedo (i.e., reflectance at diffuse incidence and observation) of infinite or semi-infinite layer $r_{\infty}$, the following approximations were tested:

(a) GKM approximation: 


$$
r_{\infty}=\frac{1-\omega_{0} F-\sqrt{\left(1-\omega_{0} F\right)^{2}-\left(\omega_{0} B\right)^{2}}}{\omega_{0} B},
$$

which may be derived (Barun and Ivanov, 2011) from a classical GKM equation (Gurevič, 1930; Kubelka and Munk, 1931; Kubelka, 1948)

$$
r_{\infty}=1+\frac{K}{S}-\sqrt{\frac{K}{S}\left(\frac{K}{S}+2\right)}
$$

assuming $K / a=S / b_{b}$ (Kubelka, 1948; Haltrin, 1998; Rogatkin, 2007).

(b) Rozenberg approximation (Rozenberg, 1962; Bushmakova et al., 1971; Zege et al., 1991):

$$
r_{\infty}=\exp \left[-4 \sqrt{\frac{1-\omega_{0}}{3(1-g)}}\right] .
$$

(c) GKM+Mudgett and Richards approach:

$$
r_{\infty}=\frac{8-5 \omega_{\mathrm{tr}}-4 \sqrt{\left(1-\omega_{\mathrm{tr}}\right)\left(4-\omega_{\mathrm{tr}}\right)}}{3 \omega_{\mathrm{tr}}},
$$

where $\omega \mathrm{tr}$ is the transport (reduced) single-scattering albedo defined as

$$
\omega_{\mathrm{tr}}=\frac{(1-g) \omega_{0}}{1-g \omega_{0}}=1-s^{2},
$$

where $s$ is determined by Eq. (A.5). This approximation may be easily derived from two equations: GKM equation for $r_{\infty}$ (Eq. C.2) and Mudgett and Richards $(1971,1972)$ equations obtained from the multi-flux numerical solution of RTE for $K$ and $S$ :

$$
K=2 a, \quad S=0.75 b(1-g), \quad \frac{K}{S}=\frac{8}{3} \frac{1-\omega_{0}}{(1-g) \omega_{0}}=\frac{8}{3} \frac{1-\omega_{\mathrm{tr}}}{\omega_{\mathrm{tr}}} .
$$

(d) Hulst's approximation (Hulst, 1974, 1980):

$$
r_{\infty}=\frac{(1-0.139 s)(1-s)}{1+1.170 s},
$$


where $s$ is determined by Eq. (A.5).

(e) Gordon et al.'s (1975) approximation:

$$
r_{\infty}=0.0003+0.3687 G+0.1802 G^{2}+0.0740 G^{3},
$$

where $G$ is the Gordon's parameter, defined as

$$
G=\frac{b_{b}}{a+b_{b}}=\frac{B \omega_{0}}{1-F \omega_{0}} .
$$

(f) GKM+modified $\delta-E(2)$ method (Meador and Weaver, 1979) is follows from Eq.

(C.2), where

$$
K=2 a, S=0.75 b(1-g)-0.25 a, \frac{K}{S}=\left\{\begin{array}{c}
100, \text { if } S \leq 0 \\
\frac{8\left(1-\omega_{0}\right)}{4 \omega_{0}-3 \omega_{0} g-1}, \text { otherwise }
\end{array}\right.
$$

(g) GKM+modified $\delta$-E(4) method (Meador and Weaver, 1979; Sokoletsky et al., 2013) is follows from Eq. (C.2), where parameters $K$ and $S$ are determined by:

$$
\begin{gathered}
K=a / \eta, \quad S=b(1-g) / \chi, \quad \frac{K}{S}=\frac{\chi}{\eta} \frac{1 / \omega_{0}-1}{1-g}, \\
\eta=\frac{132-55 \omega_{\mathrm{tr}}+35 \sqrt{1+2\left(1-\omega_{\mathrm{tr}}\right) / 35+121\left(1-\omega_{\mathrm{tr}}\right)^{2} / 49}}{224}, \quad \chi=\frac{2 \eta \omega_{\mathrm{tr}}(16 \eta-3)}{15 \eta^{2}-\left(1-\omega_{\mathrm{tr}}\right)(16 \eta-3)},
\end{gathered}
$$

and $\omega_{\mathrm{tr}}$ is determined by Eq. (C.5).

(h) GKM+van Gemert and Star (1987) approximation is follows from Eqs. (C.2) and (C.11), where parameters $\eta$ and $\chi$ are determined by

$$
\eta=0.25\left(3-\omega_{\mathrm{tr}}\right), \chi=\left(98-38 \omega_{\mathrm{tr}}\right) / 45,
$$

and $\omega t$ - by Eq. (C.5).

(i) Flock et al. (1989) approximation: 


$$
r_{\infty}=\frac{1-s^{2}}{1+(5 / \sqrt{3}) s+2 s^{2}},
$$

where $s$ is determined by Eq. (A.5).

(j) HKS 1 method (Hapke, 2012; Kokhanovsky and Sokoletsky, 2006b):

$$
r_{\infty}=\left\{(1-s)\left[\frac{1}{s}-\frac{0.5 \ln (1+2 s)}{s^{2}}\right], \text { if } s>0.2263\right. \text {, }
$$

where $s$ is determined by Eq. (A.5).

(k) Rogatkin (2007) model:

$$
\begin{gathered}
r_{\infty}=\frac{\beta_{1}-L}{\beta_{2}}, \quad \beta_{1}=\omega \times \frac{A-\ln F+\ln \left\{1-\omega+\sqrt{\omega^{2}-B^{2} \exp (-2 A)}\right\}}{\sqrt{\omega^{2}-B^{2} \exp (-2 A)}}, \\
\beta_{2}=\frac{B \exp (-A)}{\omega} \beta_{1}, \quad L=\sqrt{\beta_{1}^{2}-\beta_{2}^{2}}, \quad \omega=\frac{1-(1-2 B) \exp (-A)}{2}, \quad A=\frac{1 / \omega_{0}-1}{F} .
\end{gathered}
$$

(I) GKM+Yudovsky and Pilon (2009) model is follows from Eqs. (C.2) and (C.11), where parameters $\eta$ and $\chi$ are determined by

$$
\begin{gathered}
\xi=\sqrt{\frac{47}{52}+\frac{31}{49} \omega_{\mathrm{tr}}-\frac{49}{54} \omega_{\mathrm{tr}}^{2}-\frac{17}{27} \omega_{\mathrm{tr}}^{3}}, \phi=\frac{\xi+\ln (1-\xi)}{\xi-\ln (1+\xi)}, \\
\eta=\left\{\begin{array}{c}
0.5, \text { if } \xi<0.01 \\
\frac{(\phi-1)\left(1-\omega_{\mathrm{tr}}\right)}{(\phi+1) \xi}, \text { otherwise }
\end{array}, \quad \chi=\left\{\begin{array}{c}
4 / 3, i f \xi<0.01 \\
-\frac{0.5 \omega_{\mathrm{tr}}(\phi-1 / \phi)}{\xi}, \text { otherwise }
\end{array}\right.\right.
\end{gathered}
$$

and $\omega$ tr is determined by Eq. (C.5).

(m) GKM+Sokoletsky et al. (2013) model is follows from Eqs. (C.2) and (C.11), where parameters $\eta$ and $\chi$ are determined by

$$
\begin{gathered}
\eta=1-0.6864 \omega_{\mathrm{tr}}-0.1727 \omega_{\mathrm{tr}}^{2}+0.6783 \omega_{\mathrm{tr}}^{3}-0.3196 \omega_{\mathrm{tr}}^{4}, \\
\chi=3.321-3.495 \omega_{\mathrm{tr}}+1.777 \omega_{\mathrm{tr}}^{2}-0.2670 \omega_{\mathrm{tr}}^{3},
\end{gathered}
$$

and $\omega \mathrm{tr}$ is determined by Eq. (C.5). 
n) GKM+modified Sandoval and Kim (2014) approximation is follows from Eq. (C.2), where parameters $K$ and $S$ are determined by: Eq. (C.2) and

$$
K=2 a, S=0.75 c(1-g)-a, \frac{K}{S}=\left\{\begin{array}{c}
100, \text { if } S \leq 0 \\
\frac{8\left(1-\omega_{0}\right)}{4 \omega_{0}-3 g-1}, \text { otherwise }
\end{array} .\right.
$$

Analysis shows (Figs. C.1 and C.2) that various models may be more efficient at one set of parameters, but worse with others. For example, the Rozenberg, GKM+modified $\delta-E(2), G K M+m o d i f i e d ~ \delta-E(4)$, Rogatkin (2007), GKM+Yudovsky and Pilon (2009), and GKM+modified Sandoval and Kim (2014) models generally yield more accurate values of $r_{\infty}$ with increasing $\omega_{0}$, independently of $p(\theta)$. In contrast, the GKM, Gordon et al. (1975), and Flock et al. (1989) models deteriorate with $\omega_{0}$, at any $p(\theta)$. The other models do not reveal such clear behavior, i.e., they may give both better or worse results with increasing $\omega_{0}$. In any case, only five models [GKM+Mudgett and Richards (1971, 1972); Hulst (1974, 1980); GKM+van Gemert and Star (1987); HKS 1; and Sokoletsky et al. (2013)] yield an accuracy better than $10 \%$ at any $p(\theta)$ and $\omega_{0}$. Note especially the model of Hulst, which gives values of $r_{\infty}$ with accuracy better than $1.5 \%$ under any conditions. 

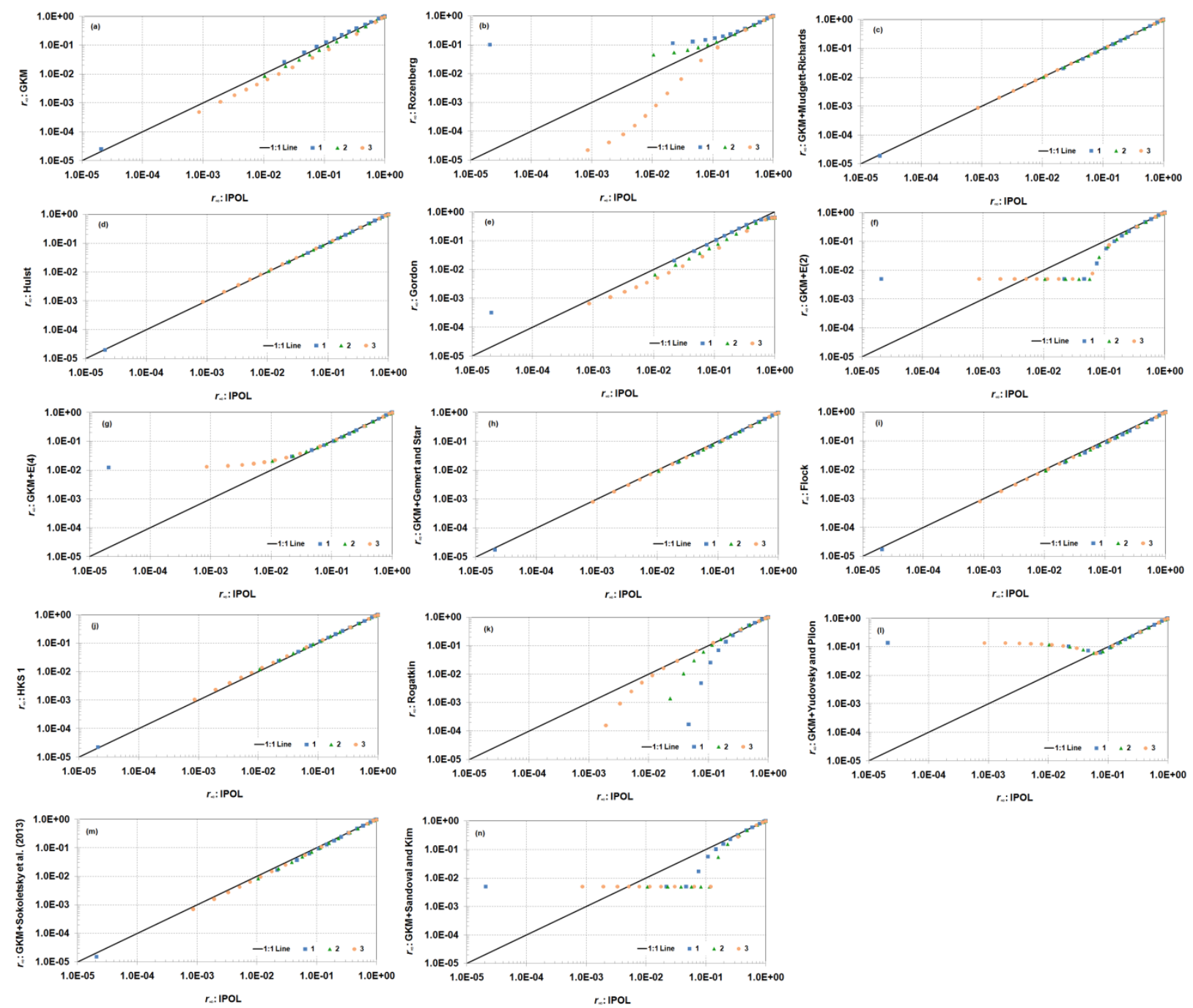

Fig. C.1. A comparison of the spherical albedo $r_{\infty}$ computed by analytical methods with the $r_{\infty}$ derived from the numerical computations (IPOL) for scattering phase functions \#1 to 3 (Table 2 and legends here) and $\omega_{0}$ varied from 0.0001 to 0.9999 . 

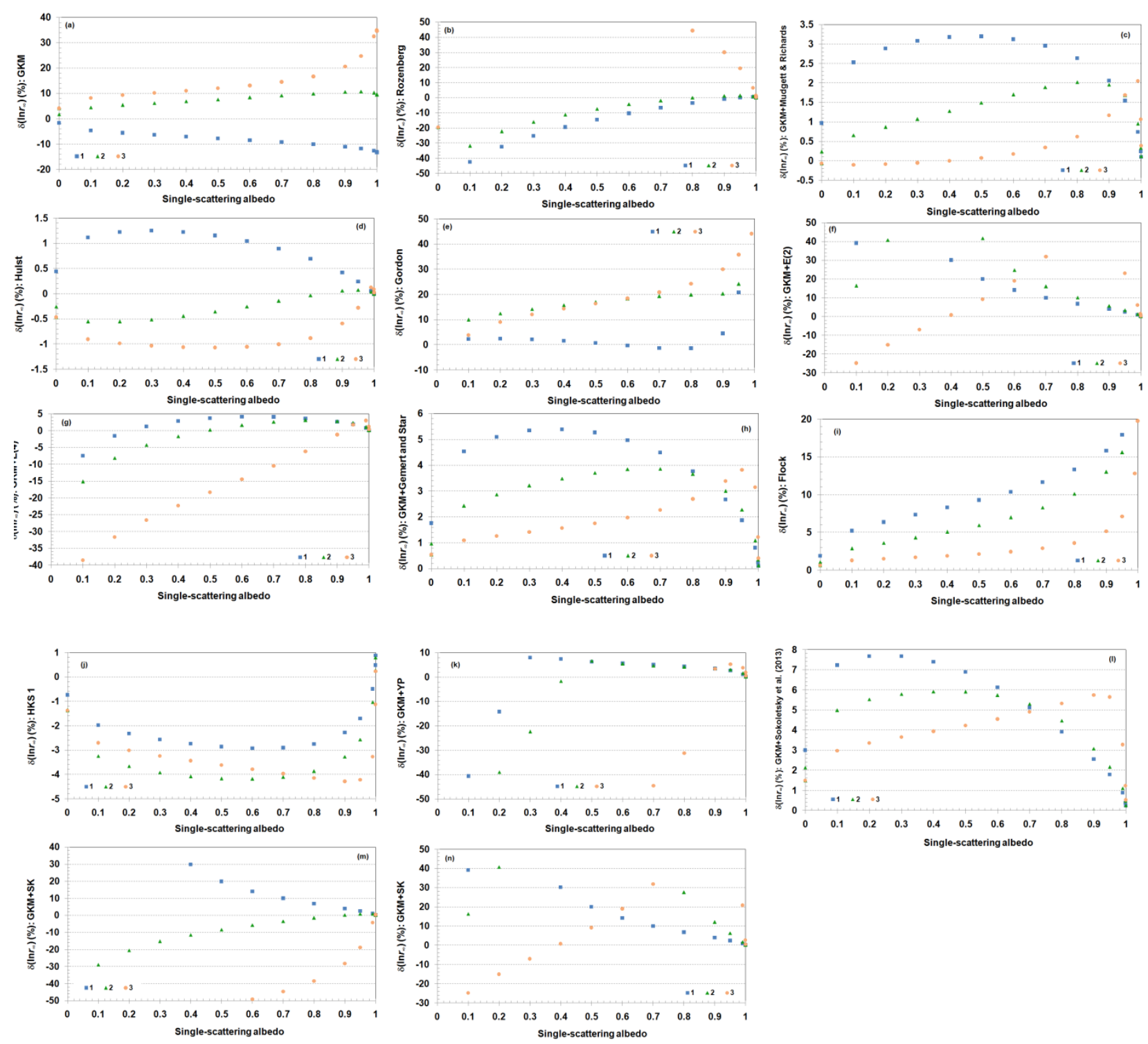

Fig. C.2. Relative logarithmic error of the spherical albedo $r_{\infty}$ computed by analytical methods relative to the $r_{\infty}$ derived from the numerical computations (IPOL) for scattering phase functions \#1 to 3 (Table 2 and legends here) and wo varied from 0.0001 to 0.9999. Note that $\delta\left(\ln r_{\infty}\right)=100 \%\left(\ln \tilde{r}_{\infty}-\ln r_{\infty}\right) / \ln r_{\infty}$, where $\tilde{r}_{\infty}$ and $r_{\infty}$ are the spherical albedos computed by analytical methods and IPOL, respectively.

\section{Appendix D. Modeling the plane albedo of infinite or semi-infinite layer}


For the plane albedo (i.e., at direct incidence and diffuse observation) of infinite or semi-infinite layer $R_{p \infty}$, the following approximations were tested:

a) Exponential approximation (Bushmakova et al., 1971; Kokhanovsky and Sokoletsky, 2006b): Eq. (A.5) and

$$
R_{\mathrm{p} \infty}\left(\mu_{\mathrm{i}}\right)=\exp \left[-\frac{4 \sqrt{3}}{7}\left(1+2 \mu_{\mathrm{i}}\right) s\right] .
$$

b) Gordon et al.'s (1975) approximation: Eq. (C.9) and

$$
R_{\mathrm{p} \infty}\left(\mu_{\mathrm{i}}\right)=\left(0.0001+0.3244 G+0.1425 G^{2}+0.1308 G^{3}\right) / \mu_{\mathrm{i}} .
$$

c) HKS 2 (or extended Hapke) approximation (Kokhanovsky and Sokoletsky, 2006b; Sokoletsky et al., 2013): Eq. (A.5) and

$$
\begin{gathered}
R_{\mathrm{p}_{\infty}}=\Phi\left(\zeta_{\mathrm{i}}\right) \frac{1-s}{1+2 \mu_{\mathrm{i}} s}, \Phi\left(\zeta_{\mathrm{i}}\right)=\exp \left\{\left(A_{1} \zeta_{\mathrm{i}}+A_{2} \zeta_{\mathrm{i}}^{2}\right) s+\left(A_{3} \zeta_{\mathrm{i}}+A_{4} \zeta_{\mathrm{i}}^{2}\right) s^{2}\right\}, \\
A_{\mathrm{j}}=\sum_{\mathrm{k}=1}^{3} \alpha_{\mathrm{jk}} g^{\mathrm{k}-1}, \quad \zeta_{\mathrm{i}}=\mu_{\mathrm{i}}-0.5, \quad \alpha_{\mathrm{jk}}=\left(\begin{array}{ccc}
-0.991 & 3.139 & -1.874 \\
1.435 & -4.294 & 2.089 \\
0.719 & -5.801 & 2.117 \\
-0.509 & 0.418 & 3.360
\end{array}\right) .
\end{gathered}
$$

d) Replacement method (Hulst, 1974, 1980; Sokoletsky et al., 2013): Eqs. (A.5), (C.7), and

$$
\begin{aligned}
R_{\mathrm{p} \infty}\left(\mu_{\mathrm{i}}\right) & =r_{\infty} \exp \left\{\left[-3.599 \ln (1-s)-0.550 \ln ^{2}(1-s)+0.0416 \ln (1-g) \ln (1-s)\right]\right. \\
& \left.\times\left[\left(1-\mu_{\mathrm{i}}\right)^{2}-\left(1-\mu_{\mathrm{ef}}\right)^{2}\right]\right\},
\end{aligned}
$$

where $\mu_{\text {ef }}$ is an effective cosine of incidence angle [at which $R_{\mathrm{p} \infty}\left(\mu_{\mathrm{ef}}\right)=r_{\infty}$ ] estimated by

$$
\mu_{\mathrm{ef}}=\cos \left(48^{\circ}+14.12 s-22.77 s^{2}+19.24 s^{3}\right) \text {. }
$$

Figs. D.1-D.4 compare $R_{p \infty}$ computed by IPOL and analytical methods at selected values $\theta_{\mathrm{i}}\left(0^{\circ}, 30^{\circ}, 60^{\circ}\right.$, and $\left.89.99^{\circ}\right)$ while Figs. D.5-D.8 demonstrate corresponding 
relative logarithmic error for the analytical model-based $R_{p \infty}$ relative to the $R_{p \infty}$ derived from the IPOL's calculations for scattering phase functions \#1 to 3 and $\omega_{0}$ varied from 0.0001 to 0.9999 .
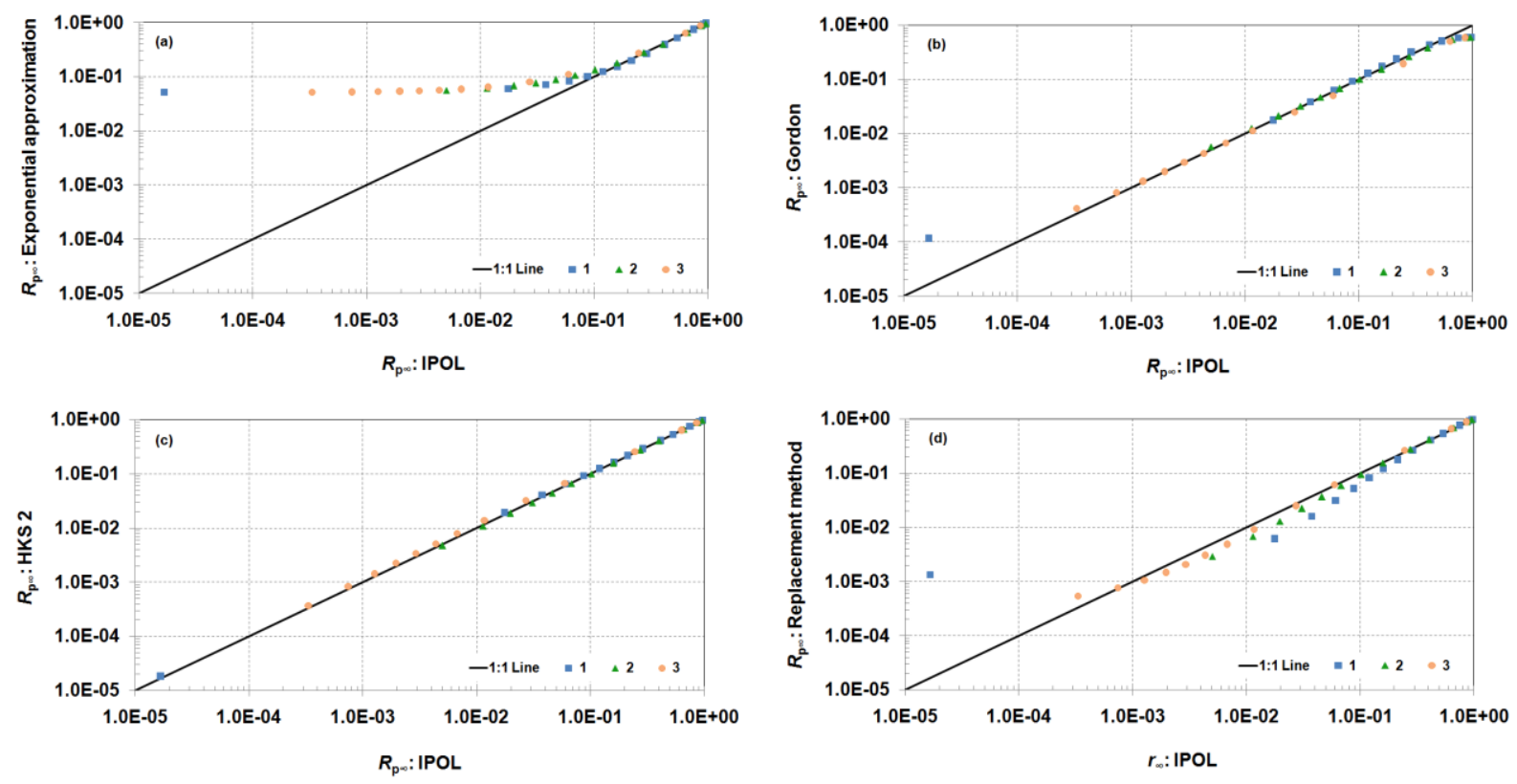

Fig. D.1. A comparison of the plane albedo $R_{p \infty}$ at $\theta_{i}=0^{\circ}$ computed by analytical methods with the $R_{p \infty}\left(0^{\circ}\right)$ derived from the numerical computations (IPOL) for scattering phase functions \#1 to 3 (Table 2) and wo varied from 0.0001 to 0.9999 . 

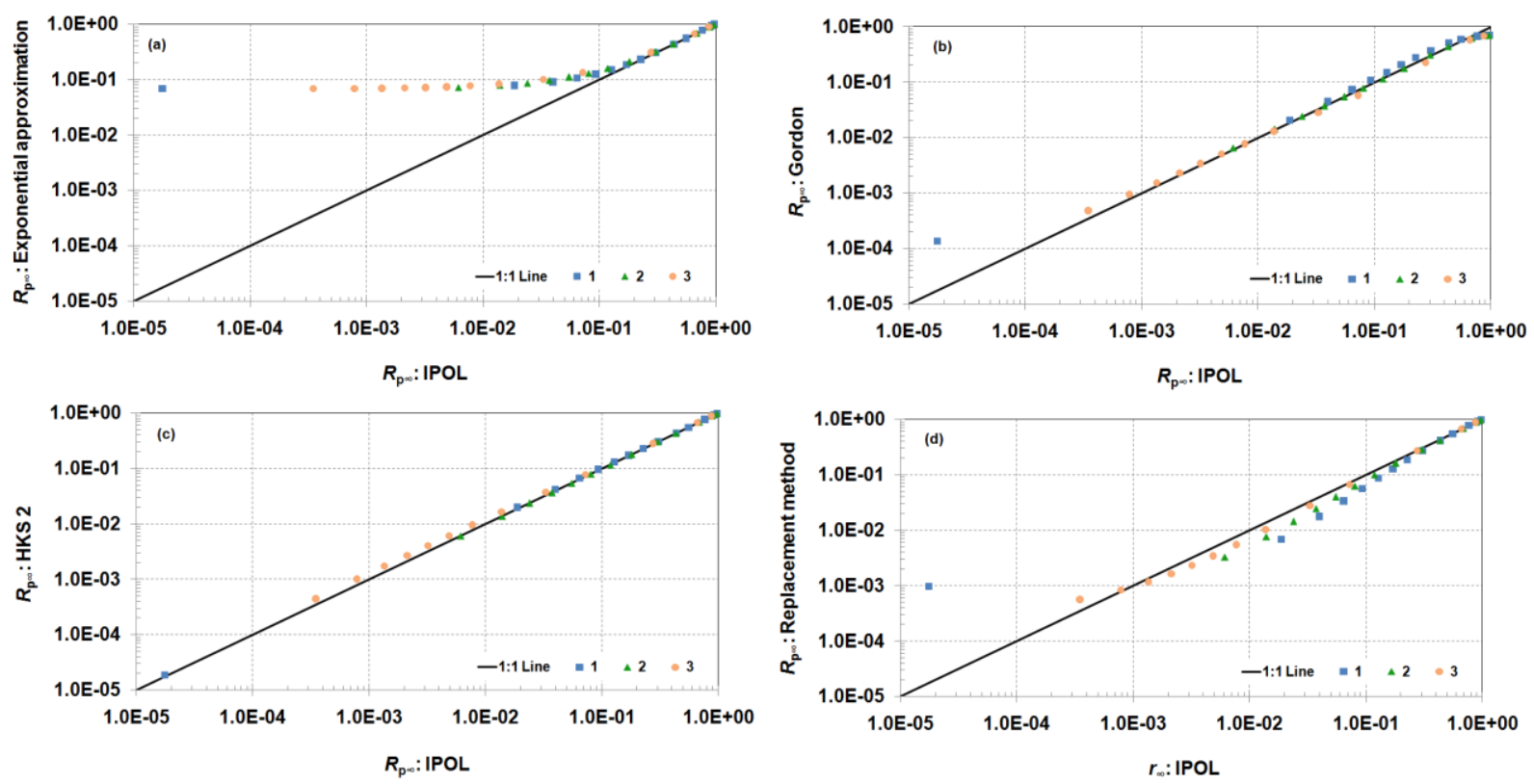

Fig. D.2. The same as Fig. D.1, but for $R_{p \infty}\left(30^{\circ}\right)$.
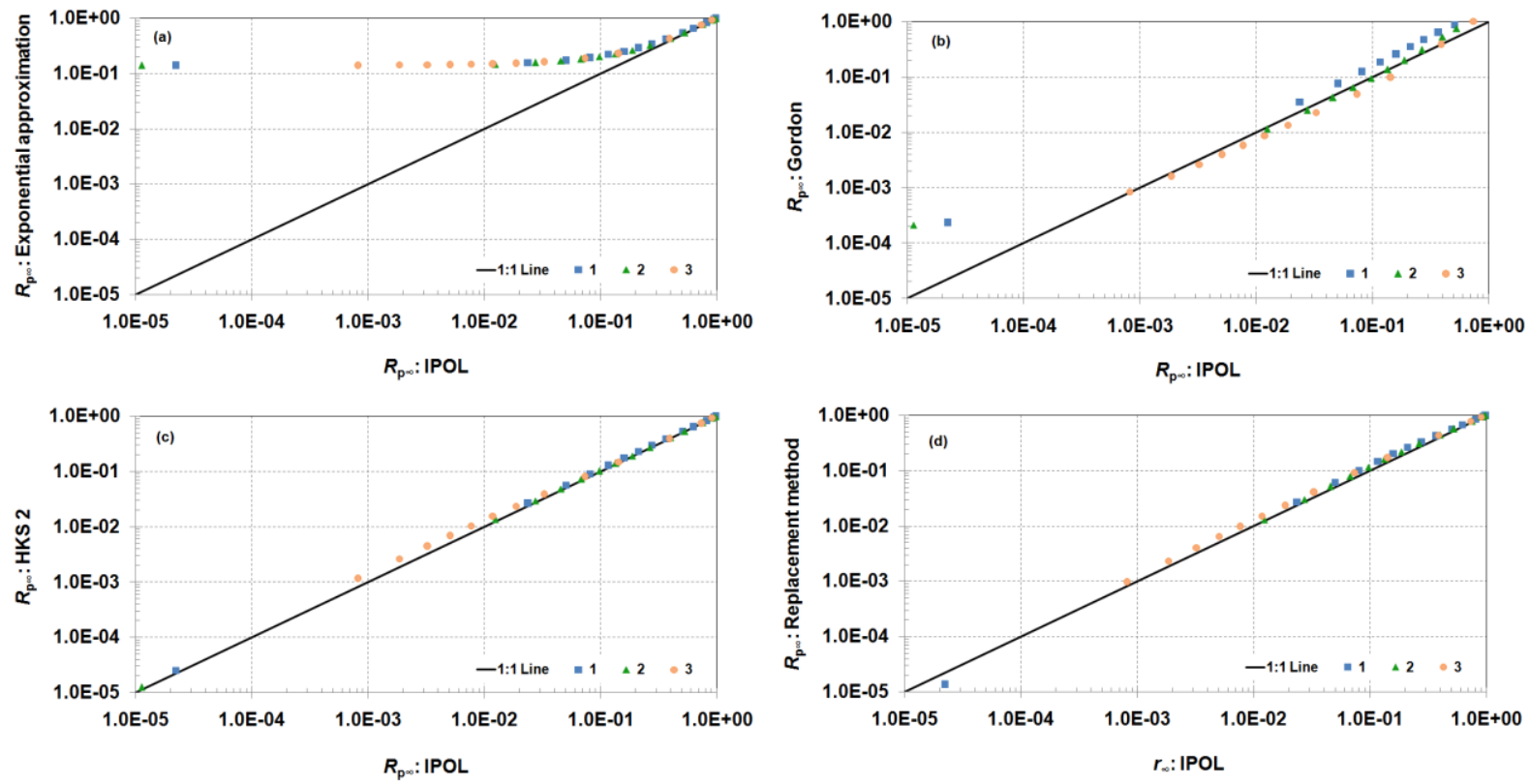

Fig. D.3. The same as Fig. D.1, but for $R_{p \infty}\left(60^{\circ}\right)$. 

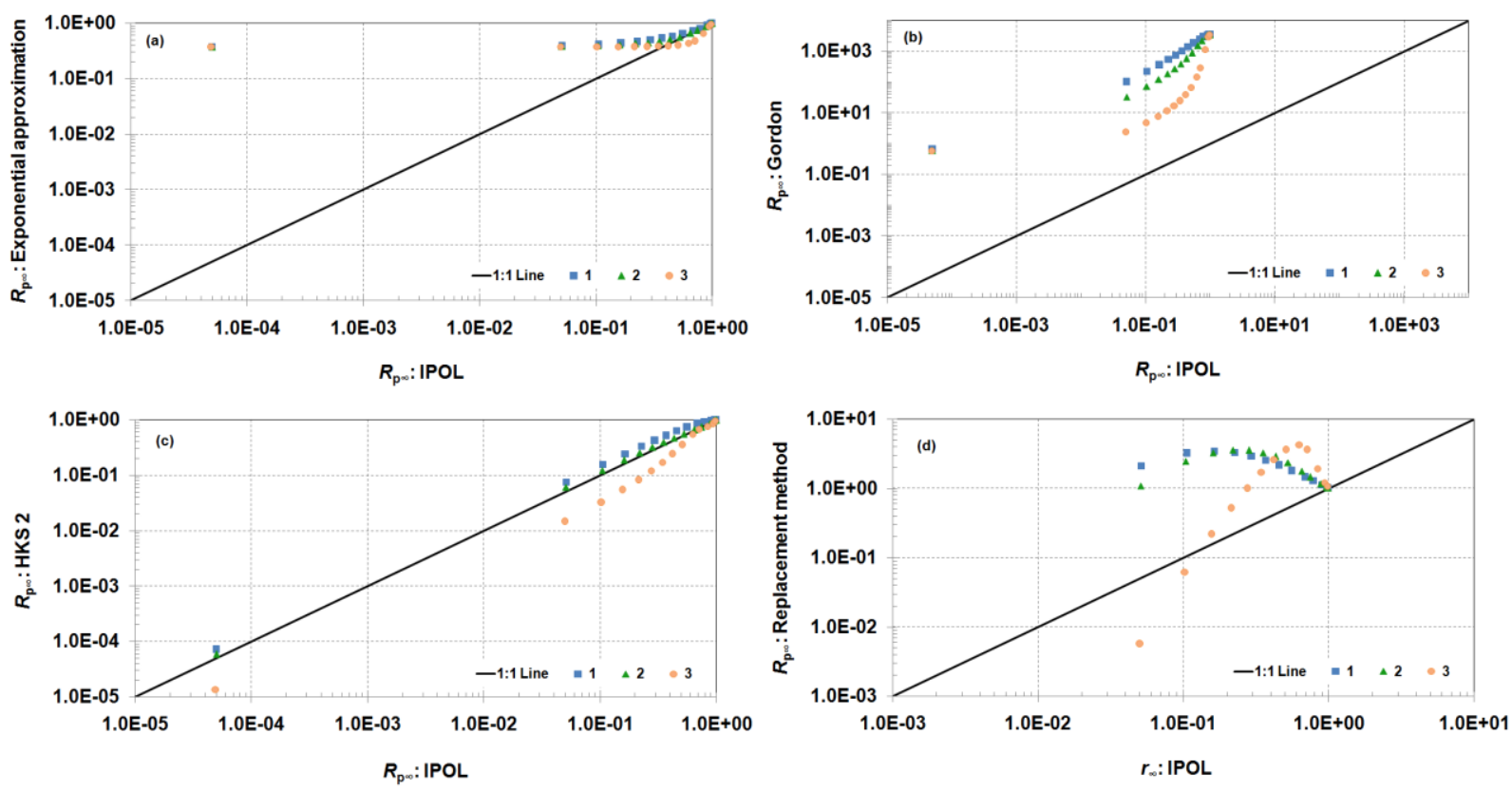

Fig. D.4. The same as Fig. D.1, but for $R_{\mathrm{p} \infty}\left(89.99^{\circ}\right)$.
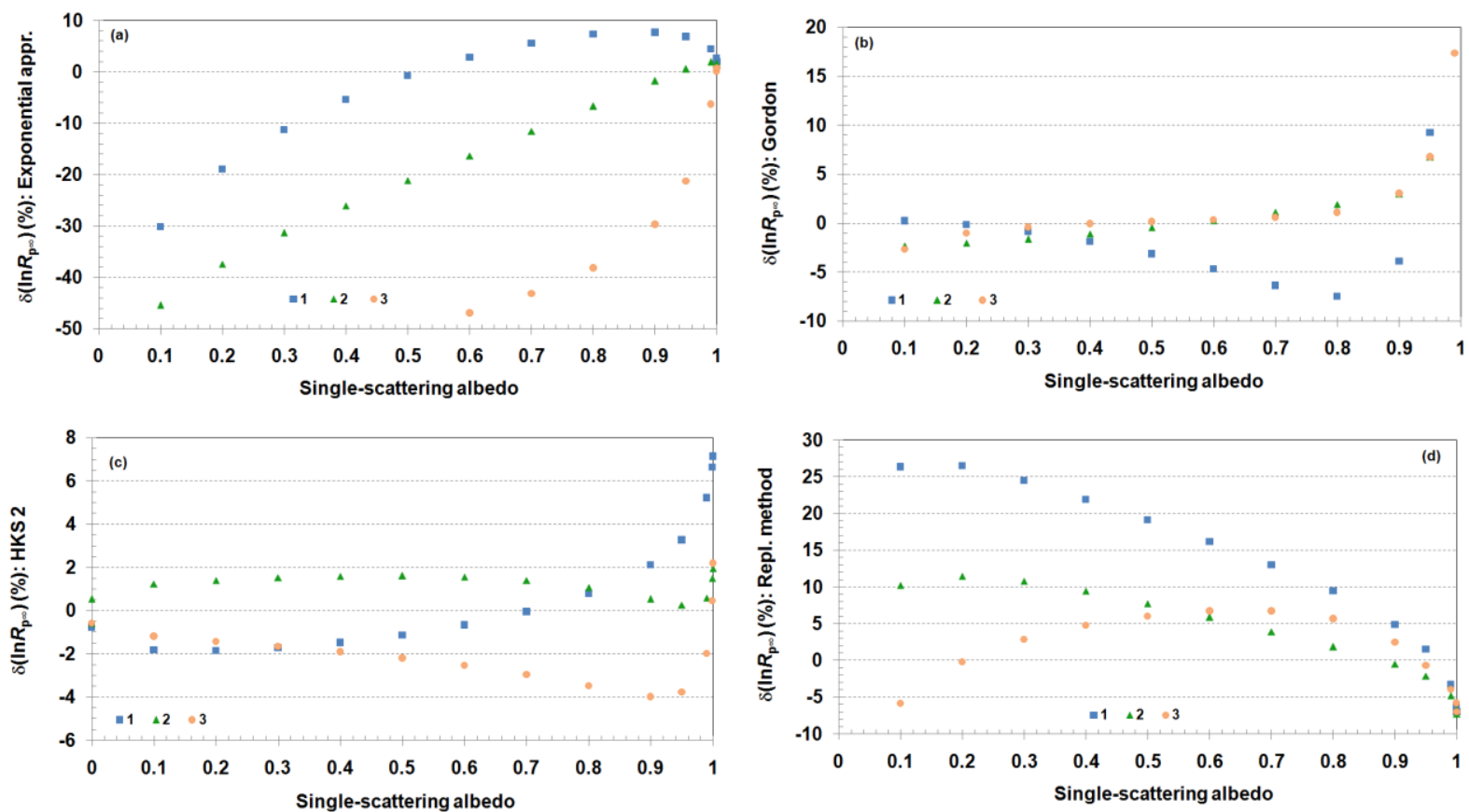

Fig. D.5. Relative logarithmic error of the plane albedo $R_{p \infty}$ at $\theta_{i}=0^{\circ}$ computed by analytical methods relative to the $R_{p \infty}\left(0^{\circ}\right)$ derived from the numerical computations (IPOL) for scattering phase functions \#1 to 3 (Table 2) and $\omega_{0}$ varied from 0.0001 to 0.9999 . 

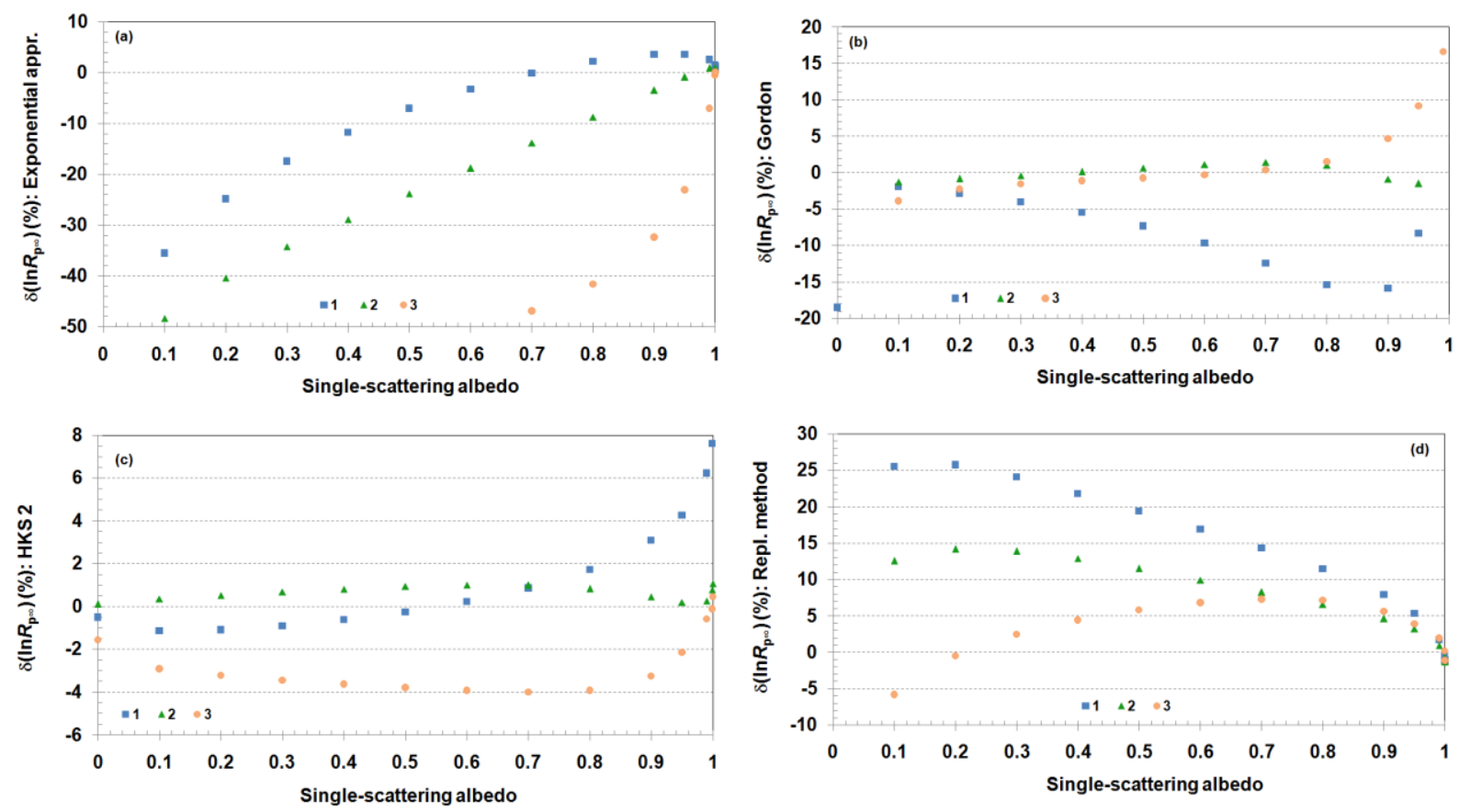

Fig. D.6. The same as Fig. D.5, but for $R_{\mathrm{p} \infty}\left(30^{\circ}\right)$.
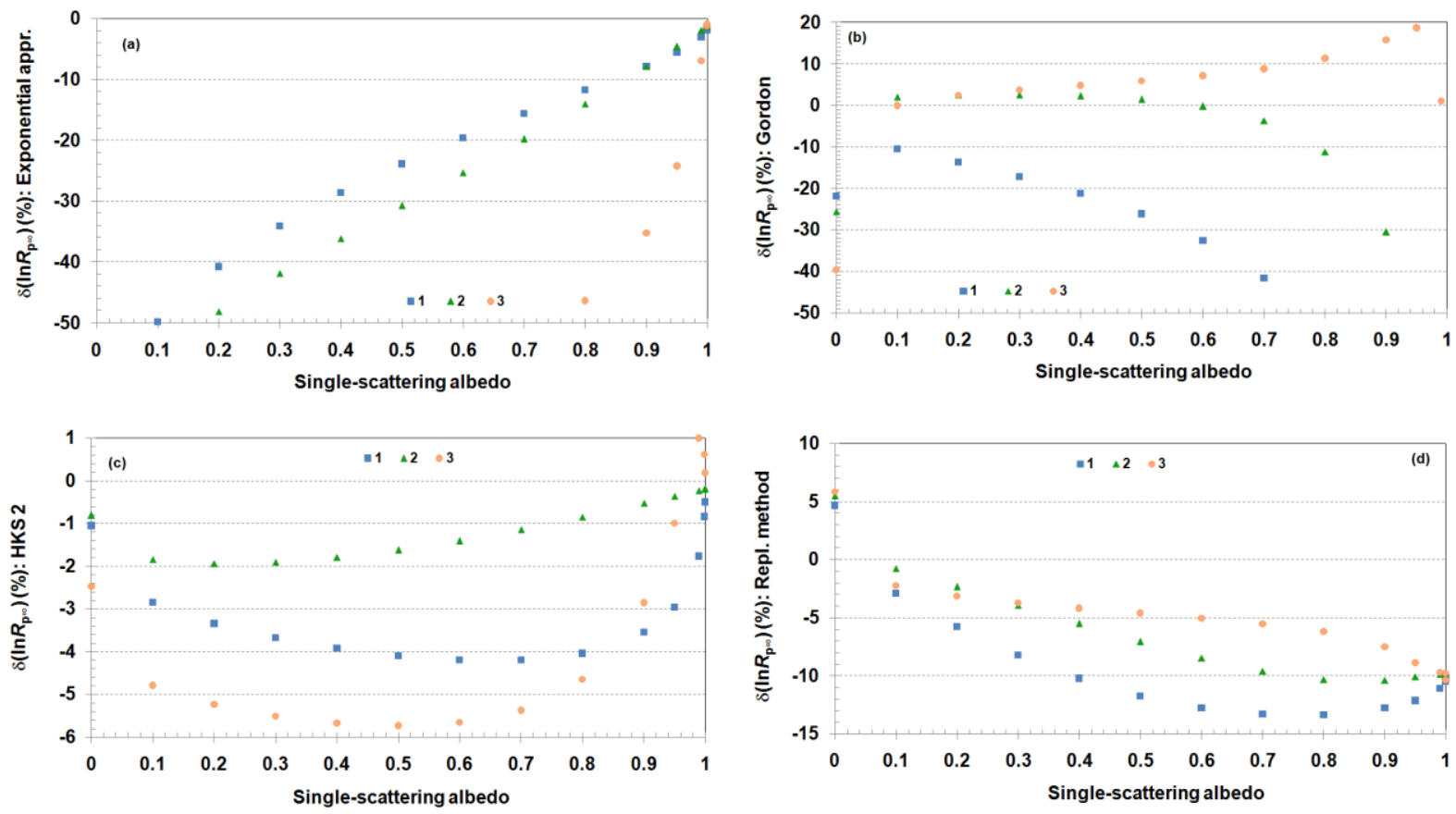

Fig. D.7. The same as Fig. D.5, but for $R_{p \infty}\left(60^{\circ}\right)$. 

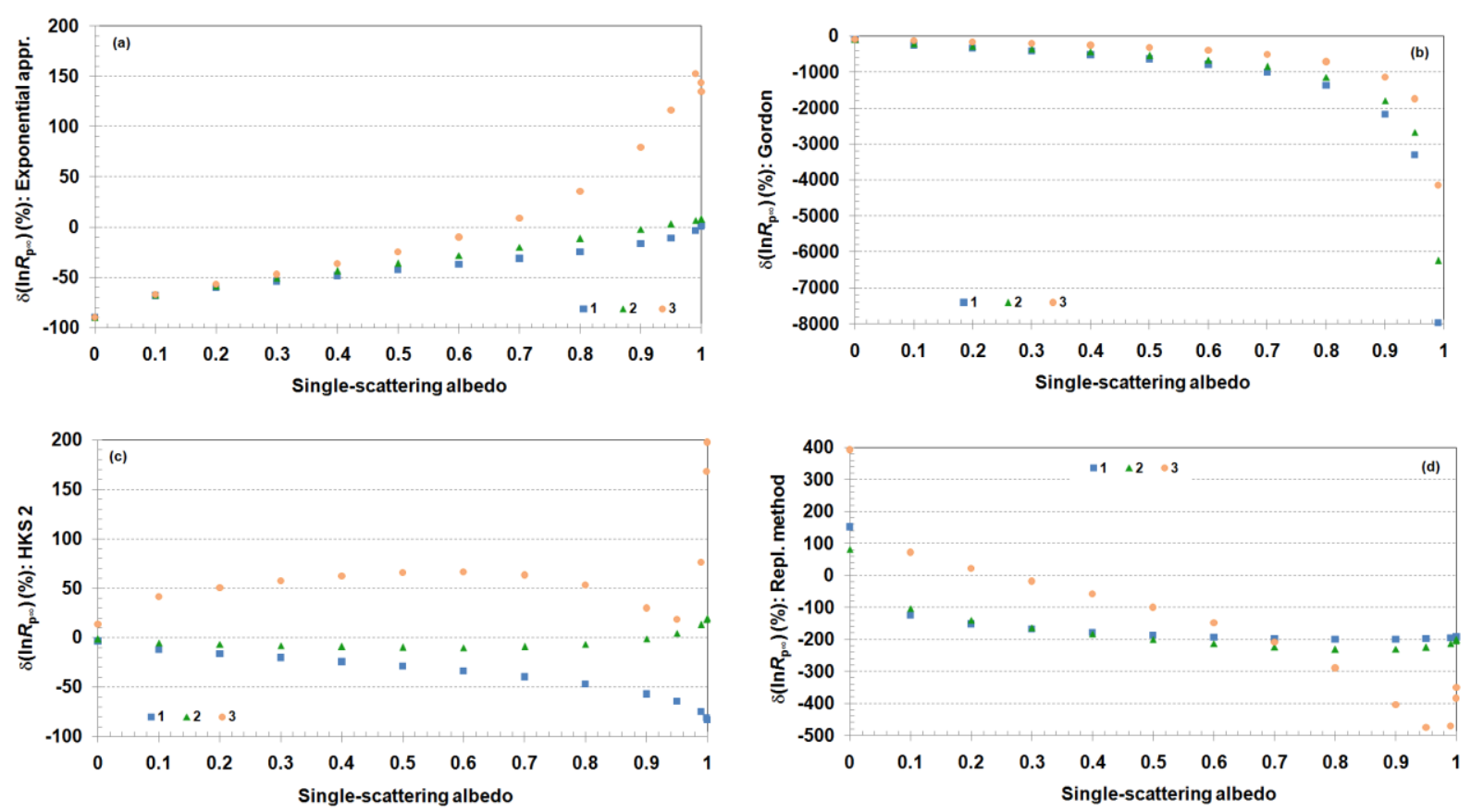

Fig. D.8. The same as Fig. D.5, but for $R_{\mathrm{p} \infty}\left(89.99^{\circ}\right)$.

As these results have shown, the exponential and replacement models yield generally better accuracy with increasing $\omega_{0}$, while the Gordon et al.'s (1975) model normally deteriorates. The quality of $R_{p \infty}$ generally becomes lower with increasing $\theta \mathrm{i}$ for any models; moreover, Gordon et al.'s (1975) and replacement models completely fail at incident angles close to $90^{\circ}$. The best results demonstrated were generally those of the HKS 2 model with a logarithmic error $<10 \%$ at $\theta_{i} \leq 60^{\circ}$ and any $p(\theta)$ and $\omega_{0 .}$ However, at very large incoming angles, the situation may be changed in favor of the exponential model. For example, at $\theta_{i}=89.99^{\circ}$, the last model is superior for $\omega_{0} \geq 0.7$, $\omega_{0} \geq 0.95$, and $0.3 \leq \omega_{0} \leq 0.8$ for the $p(\theta) \# 1-3$, respectively. For smaller values of $\omega_{0}$, the best results generally demonstrated by the HKS 2 model at this angle. 


\section{Acknowledgements}

The authors thank Alexander Kokhanovsky (EUMETSAT) and Sergey Korkin (NASA GSFC) for providing their results on numerical solution (by the SORD and IPOL codes) of radiative transfer equation. The authors would like also to acknowledge anonymous reviewer for constructive comments and recommendations which helped to improve the readability and quality of this paper. The research leading to these results was funded by the National Natural Science Foundation of China, NSFC (grants No. 41271375 and 41371346). All input and output data used for modelling computations are available upon requirement.

\section{References}

Aas E. Two stream irradiance model for deep waters. Appl Opt 1987;26(11):2095-2101.

Aas E, Højerslev NK. Analysis of underwater radiance observations: Apparent optical properties and analytic functions describing the angular radiance distribution. $J$ Geophys Res 1999;104(C4):8015-8024.

Ambartsumian VA. A new method of calculation of the light scattering in turbid medium. Izvestiya Acad. Sci USSR, Ser Geograph and Geophys 1942;3:97-103 (in Russian). Barun VV, Ivanov AP. Optical parameters of disperse medium with large absorbing and scattering inclusions. Opt Spectr 2011;110(3):418-424.

Beer A. Bestimmung der Absorption des rothen Lichts in farbigen Flüssigkeiten ["Determination of the absorption of red light in colored liquids"]. Annalen der Physik und Chemie 1852;86:78-88 (in German). 
Ben-David A. Multiple-scattering transmission and an effective average photon path length of a plane-parallel beam in a homogeneous medium. Appl Opt 1995;34(15):2802-2810.

Ben-David A. Multiple-scattering effects on differential absorption for the transmission of a plane-parallel beam in a homogeneous medium. Appl Opt 1997;36(6):1386-1398.

Berwald J, Stramski D, Mobley CD, Kiefer DA. Influences of absorption and scattering on vertical changes in the average cosine of the underwater light field. Limnol Oceanogr 1995;40(8):1347-1357.

Bissett WP, Carder KL, Walsh JJ, Dieterle DA. Carbon cycling in the upper waters of the Sargasso Sea: II. Numerical simulation of apparent and inherent optical properties. Deep-Sea Res 1999;46:271-317.

Bouguer P. Essai d'optique sur la gradation de la lumière. Paris, France: Claude Jombert; 1729 (in French).

Budak VP, Korkin SV. On the solution of a vectorial radiative transfer equation in an arbitrary three-dimensional turbid medium with anisotropic scattering. J Quant Spectrosc Radiat Transfer 2008;109(2):220-234.

Bushmakova OV, Zege EP, Katsev IL. On asymptotic equations for brightness coefficients of optically thick light scattering layers. Doklady Acad Sci BSSR 1971;15:309-311.

Carder KL, Chen FR, Hawes SK. Instantaneous Photosynthetically Available Radiation and Absorbed Radiation by Phytoplankton. Algorithm Theoretical Basis Documents (ATBD 20), Version 5. April, 26, 1999. St. Petersburg, Florida, USA. 
Cornet JF, Dussap CG, Dubertret G. A structured model for simulation of cultures of the cyanobacterium Spirulina platensis in photobioreactors: I. Coupling between light transfer and growth kinetics. Biotechnol. Bioeng 1992;40(7):817-825.

Deirmendjian A. Electromagnetic scattering on spherical polydispersions. New York, USA: Elsevier, 1969.

Doicu A, Traumann T, Schreier F. Numerical regularization for atmospheric inverse problems. Berlin, Heidelberg, Germany: Springer-Verlag, 2010.

Dubovik O, Holben B, Eck TF, Smirnov A, Kaufman YJ, King MD, Tanre D, Slutsker I. Variability of absorption and optical properties of key aerosol types observed in worldwide locations. J Atm Sci 2002;59(3):590-608.

Emde C, Barlakas V, Cornet C, Evans F, Korkin S, Ota Y, Labonnote LC, Lyapustin A, Macke A, Mayer B, Wendisch M. IPRT polarized radiative transfer model intercomparison project - Phase A. J Quant Spectrosc Radiat Transfer 2015;164:836.

Flock ST, Patterson MS, Wilson BC, Wyman DR. Monte Carlo modeling of light propagation in highly scattering tissues-l.: Model predictions and comparison with diffusion theory. IEEE Trans Biomed Eng 1989;36(12):1162-1168.

Gemert MJC. van, Star WM. Relations between the Kubelka-Munk and the transport equation models for anisotropic scattering. Lasers Life Sci 1987;1:287-298.

Gershun AA. Transmission of light through a flat layer of a light scattering medium. $\mathrm{Tr}$ Gos Opt Inst (Proc State Opt Inst) 1936;11(99):43-68.

Gordon HR. Simple calculation of the diffuse reflectance of ocean. Appl Opt 1973;12(12): 2803-2804. 
Gordon HR, Brown OB. Influence of bottom depth and albedo on the diffuse reflectance of a flat homogeneous ocean. Appl Opt 1974;13(9):2153-2159.

Gordon HR, Brown OB, Jacobs MM(1975). Computed relationships between the inherent and apparent optical properties of a flat homogeneous ocean. Appl Opt $1975 ; 14(2): 417-427$.

Gordon HR. Can the Lamber-Beer law be applied to the diffuse attenuation coefficient of ocean water? Limnol Oceanogr 1989;34(8):1389-1409.

Gurevič M. Über eine rationelle klassifikation der lichtenstreuenden medien. Physikalische Zeitschrift 1930;31:753-763 (in German).

Hapke B. Theory of reflectance and emittance spectroscopy, 2nd ed. Cambridge, UK: Cambrige Univ Press; 2012.

Haltrin VI. Self-consistent approach to the solution of the light transfer problem for irradiances in marine waters with arbitrary turbidity, depth, and surface illumination.

I. Case of absorption and elastic scattering. Appl Opt 1998;37(18):3773-3784.

Hébert M, Machizaud J. Spectral reflectance and transmittance of stacks of nonscattering films printed with halftone colors. J Opt Soc Am A 2012;29(11):24982508.

Hecht HG. The interpretation of diffuse reflectance spectra. J Res Natl Bur Stand 1976;80A(4):567-583.

Henyey LC, Greenstein JL. Diffuse radiation in the galaxy. Astrophys. J 1941,93:70-83.

Hirata T. Irradiance inversion theory to retrieve volume scattering function of seawater. Appl Opt 2004;42(9):1564-1573. 
Højerslev NK. Analytic remote-sensing optical algorithms requiring simple and practical field parameter inputs. Appl Opt 2001; 40(27):4870-4874.

Hulst HC van de. The spherical albedo of a planet covered with a homogeneous cloud layer. Astron Astrophys 1974;35:209-214.

Hulst HC van de. Multiple light scattering, vol. 2. London, UK: Academic Press; 1980.

Judd DB. Color in business, science and industry, 3rd ed. Wiley, New York, USA: WileyInterscience; 1975.

Kattawar GW, Plass GN. Asymptotic radiance and polarization in optically thick media: ocean and clouds. Appl Opt 1976;15(12):3166-3178.

King MD, Harshvardhan. Comparative accuracy of selected multiply scattering approximations. J Atm Sci 1986;43(8):784-801.

Kirk JTO. Volume scattering function, average cosines, and the underwater light field. Limnol Oceanogr 1991;36(3):455-467.

Kirk JTO. Multiply scattering of a photon flux: implications for the integral average cosine of the underwater light field. Appl Opt 1999;38(15):3134-3140.

Kirk JTO. Light and photosynthesis in Aquatic Ecosystems, 3rd ed. Cambrige, UK: Cambridge University Press; 2011.

Kokhanovsky AA. Cloud Optics. Dordrecht, The Netherlands: Springer; 2006.

Kokhanovsky AA, Sokoletsky LG. Reflection of light from semi-infinite absorbing turbid media. Part 1: Spherical albedo. Color Res Appl 2006a;31(6):491-497.

Kokhanovsky AA, Sokoletsky LG. Reflection of light from semi-infinite absorbing turbid media. Part 2: Plane albedo and reflection function. Color Res Appl 2006b;31(6):498-509. 
Kokhanovsky AA. Physical interpretation and accuracy of the Kubelka-Munk theory. $J$ Phys D Appl Phys 2007;40:2210-2216.

Kokhanovsky AA., Budak VP, Cornet C, Duan M, Emde C, Katsev IL, Klyukov DA, Korkin SV, C-Labonnote L, Mayer B, Min Q, Nakajima T, Ota Y, Prikhach AS, Rozanov VV, Yokota T, Zege EP. Benchmark results in vector atmospheric radiative transfer. J Quant Spectrosc Radiat Transfer 2010;111(12-13):1931-1946.

Korkin S, Lyapustin A, Sinyuk A, Holben B, Kokhanovsky A. Vector radiative transfer code SORD: Performance analysis and quick start guide. J Quant Spectrosc Radiat Transfer 2017;200:295-310.

Kubelka P, Munk F. Ein beitrag zur optik der farbanstriche. Zeitschrift für Technische Physik 1931;12:593-601 (in German).

Kubelka P. New contributions to the optics of intensely light-scattering material. Part I. J Opt Soc Am 1948;38(5):448-457.

Kubelka P. New contributions to the optics of intensely light-scattering material. Part II: Nonhomogeneous layers. J Opt Soc Am 1954;44(4):330-335.

Lambert $\mathrm{JH}$. Photometria sive de mensura et gradibus luminis, colorum et umbrae ["Photometry, or, On the measure and gradations of light, colors, and shade"]. Augsburg, Germany: Eberhardt Klett; 1760 (in Latin).

Leathers RA, McCormic NJ. Ocean inherent optical property estimation from irradiances. Appl Opt 1997,36(33):8685-8698.

Lee S, Kim M, Choi M, Go S, Kim J, Kim J-H, Lim H-K, Jeong U, Goo T-Y, Kuze A, Shiomi K, Tatsuya Y. Aerosol property retrieval algorithm over Northeast Asia from 
TANSO-CAI measurements onboard GOSAT. Remote Sens 2017;9(7):687. doi:10.3390/rs9070687.

Lee Z.-P, Du KP, Arnone R. A model for the diffuse attenuation coefficient of downwelling irradiance. J Geophys Res 2005a;110:C02016. doi:10.1029/2004JC002275.

Lee Z.-P, Darecki M, Carder KL, Davis CO, Stramski D, Rhea WJ. Diffuse attenuation coefficient of downwelling irradiance: An evaluation of remote sensing methods. $J$ Geophys Res 2005b;110:C02017. doi:10.1029/2004JC002573.

Lee Z-P. Synthesized dataset from IOCCG Report 5. 2006. http://www.ioccg.org/groups/OCAG_data.html.

Liu J, Schaaf C, Strahler A, Jiao Z, Shuai Y, Zhang Q, Roman M, Augustine JA, Dutton EG. Validation of Moderate Resolution Imaging Spectroradiometer (MODIS) albedo retrieval algorithm: Dependence of albedo on solar zenith angle. J Geophys Res 2009;114:D01106. doi:10.1029/2008JD0099.

Martins JV, Tanre D, Remer L, Kaufman Y, Mattoo S, Levy R. MODIS cloud screening for remote sensing of aerosols over oceans using spatial variability. Geophys Res Lett 2002; 29(12):MOD4-1 - MOD4-4.

McCormick NJ. Mathematical models for the mean cosine of irradiance and the diffuse attenuation coefficient. Limnol Oceanogr 1995;40(5):1013-1018.

Meador WE, Weaver WR. Diffusion approximation for large absorption in radiative transfer. Appl Opt 1979;18(8):1204-1208.

Mie G. (1908). Beiträge zur optik trüber medien, speziell kolloidaler metallösungen. Ann Phys 1908;25(3):377-445 (in German). 
Mobley CD. Light and water: radiative transfer in natural waters, San Diego, CA, USA: Academic Press; 1994.

Mobley C. Radiative transfer theory. The Asymptotic radiance distribution. In: Mobley C, Boss E, Roesler C. Ocean optics web book, 2017. http://www.oceanopticsbook.info/view/radiative_transfer_theory/the_ asymptotic_radiance_distribution.

Molenaar R, Bosch JJ ten, Zijp JR. Determination of Kubelka-Munk scattering and absorption coefficients by diffuse illumination. Appl Opt 1999;38(10):2068-2077 .

Morel A, Loisel H. Apparent optical properties of oceanic water: Dependence on the molecular scattering contribution. Appl Opt 1998;37(21):4765-4776.

Mudgett PS, Richards LW. Multiple scattering calculations for technology. Appl Opt 1971;10(7):1485-1502.

Mudgett PS, Richards LW. Multiple scattering calculations for technology II. J Colloid Interface Sci 1972;39(3):551-567.

Nakajima T, King MD. Asymptotic theory for optically thick layers: application to the discrete ordinates method. Appl Opt 1992;31(36):7669-7683.

Nechad B, Ruddick K. A model of diffuse attenuation of downwelling irradiance for ecosystem models. Proc. SPIE Remote Sensing of the Coastal Ocean, Land, and Atmosphere Environment; 2010:7858.

https://odnature.naturalsciences.be/downloads/publications/nechad_ruddick_2010_s pie_.pdf. doi:10.1117/12.872979.

O'Neil PV. Advanced engineering mathematics, SI, 8th ed. Boston, MA, USA: Cengage Learning; 2017. 
Pan X, Zimmerman RC. Modeling the vertical distributions of downwelling plane irradiance and diffuse attenuation coefficient in optically deep waters. J. Geophys. Res 2010;115:C08016. doi:10.1029/2009JC006039.

Pierce PE, Marcus RT. Radiative transfer theory solid color-matching calculations. Color Res Appl 1997;22(2):72-87.

Pottier L, Pruvost J, Deremetz J, Cornet JF, Legrand J, Dussap CG. A fully predictive model for one-dimensional light attenuation by Chlamydomonas reinhardtii in a torus photobioreactor. Biotechnol Bioeng 2005;91(5):569-582.

Prahl SA. (1995). The adding-doubling method. Ch. 5 in Optical-thermal response of laser irradiated tissue. Eds. Welch AJ, Gemert MJC van. New York, USA: Springer; 1995. Pp. 101-129. http://omlc.org/ prahl/pubs/pdf/prahl95c.pdf.

Rogatkin DA. A specific feature of the procedure for determination of optical properties of turbid biological tissues and media in calculation for noninvasive medical spectrophotometry. Biomed. Eng 2007;41(2):59-65.

Rozanov VV, Rozanov AV, Kokhanovsky AA, Burrows JP. Radiative transfer through terrestrial atmosphere and ocean: Software package SCIATRAN. J. Quant. Spectrosc. Radiat. Transfer 2014;133:13-71.

Rozenberg GV. Light characteristics of thick layers of a weakly absorbing scattering medium. Doklady Acad. Sci USSR 1962;145:775-777 (in Russian).

Sandoval C, Kim AD. Deriving Kubelka-Munk theory from radiative transfer. J Opt Soc Am A 2014;31(3):628-636.

Sathyendranath S, Platt T. Analytic model of ocean color. Appl Opt 1997;36(12):26202629. 
Schuster A. Radiation through a foggy atmosphere. Astrophys J 1905;21(1):1-22. Reprinted in Menzel DH. Selected Papers on the Transfer of Radiation. New York, USA: Dover; 1966.

Schwarzschild K. Über das Gleichgewicht der Sonnenatmosphären (On the equilibrium of the solar atmosphere). Nachrichten von der Königl. Gesellschaft der Wissenschaften zu Göttingen, Mathematisch-Physikalische Klasse 1906;195:41-53 (in German).

Shifrin KS. Physical optics of ocean water. New York, USA: AIP Translation Series. American Institute of Physics (Russian edition, 1983); 1988.

Siegel DA, Dickey TD. On the parameterization of irradiance for open ocean photoprocesses. J Geophys Res 1987;92(C13):14,648-14,662.

Sobolev VV. Radiation energy transfer in stellar and planetary atmospheres. Moscow, USSR: GITTL; 1956 (in Russian).

Sobolev VV. The diffusion of radiation in a medium of finite optical thickness. Soviet Astron 1957;1:332-345 (Published by Am Inst Phys).

Sobolev VV. Light Scattering in planetary atmospheres. Oxford, UK: Pergamon; 1975.

Sokoletsky L, Dubinsky Z, Shoshany M, Stambler N. Estimation of phytoplankton pigment concentration in the Gulf of Aqaba (Eilat) by in situ and remote sensing single-wavelength algorithms. Int J Rem Sensing 2003;24(24):5049-5073.

Sokoletsky L. A comparative analysis of simple radiative transfer approaches for aquatic environments. Proc 2004 ENVISAT \& ERS Symp.

https://www.researchgate.net/publication/235697460_A_Comparative_Analysis_of_ Simple_Radiative_Transfer_Approaches_for_Aquatic_Environments. 
Sokoletsky LG, Kokhanovsky AA. Reflective characteristics of natural waters: The accuracy of selected approximations. Proc. Current Problems in Optics of Natural Waters (ONW-2005) Conf.

https://www.researchgate.net/publication/235697641_Reflective_Characteristics_of_ Natural_Waters_The_Accuracy_of_Selected_Approximations.

Sokoletsky LG, Nikolaeva OV, Budak VP, Bass LP, Lunetta RS, Kuznetsov VS, Kokhanovsky AA. A comparison of numerical and analytical radiative-transfer solutions for plane albedo of natural waters. J Quant Spectrosc Radiat Transfer 2009;110(13):1132-1146.

Sokoletsky LG, Kokhanovsky AA, Shen F. Comparative analysis of radiative transfer approaches for calculation of diffuse reflectance of plane-parallel light-scattering layers. Appl Opt 2013;52(35):8471-8483.

Sokoletsky LG., Shen F. Optical closure for remote-sensing reflectance based on accurate radiative transfer approximations: the case of the Changjiang (Yangtze) River Estuary and its adjacent coastal area, China. Int. J. Rem. Sensing (2014);35(11-12):4193-4224.

Sokoletsky LG, Budak VP, Shen F, Kokhanovsky AA. Comparative analysis of radiative transfer approaches for calculation of plane transmittance and diffuse attenuation coefficient of plane-parallel light scattering layers. Appl Opt 2014a;53(3):459-468.

Sokoletsky L, Yang X, Shen F. Modeling the direct to diffuse downwelling irradiance ratio based on the atmospheric spectral radiation model. Proc Ocean Optics XXII Conf. 2014b. 
https://www.researchgate.net/publication/265511168_Modeling_the_Direct_to_Diffu se_Downwelling_Irradiance_Ratio_Based_on_the_Atmospheric_Spectral_Radiation -Model.

Sokoletsky L, Budak V. A comparative analysis of existing models for diffuse attenuation coefficient of downwelling irradiance with a special emphasis on natural waters. Proc 13th Annual Meeting Asia Oceania Geosciences Society (AOGS). 2016a.

https://www.researchgate.net/305882284_A_Comparative_Analysis_of_Existing _Models_for_Diffuse_Attenuation_Coefficient_of_Downwelling_Irradiance_with_a_S pecial_Emphasis_on_Natural_Waters.

Sokoletsky LG, Budak VP. Looking for the best light transmission model for the Earth's atmosphere and natural waters. Proc Ocean Optics XXIII Conf. 2016b.

https://www.researchgate.net/publication/308915113_Looking_for_the_best _light_transmission_model_for_the_Earth's_atmosphere_and_natural_waters.

Sokoletsky L., Budak V. Looking for the best light transmission model for the Earth's atmosphere and natural waters. MESE $2017 ; 3(6): 365-372$.

Sun X, Han Y, Shi X. Application of asymptotic theory for computing the reflection of optically thick clouds. J Opt A Pure Appl Opt 2006;8:1074-1079.

Tuckerman LB. On the intensity of the light reflected from or transmitted through a pile of plates. J Opt Soc Am 1947;37(10):818-825.

Twardowski MS, Boss E., Macdonald JB, Pegau WS, Barnard AH, Zaneveld JRV. A model for estimating bulk refractive index from the optical backscattering ratio and 
the implications for understanding particle composition in case I and case II waters. J Geophys Res 2001;106(C7):14,129-14,142.

Twardowski MS, Claustre H, Freeman SA, Stramski D, Huot Y. Optical backscattering properties of the "clearest" natural waters. Biogeosciences 2007;4:1041-1058.

Ueno S. The invariant imbedding method for transport problems II. Resolvent in photon diffusion equation. J Math Anal And Appl 1961;3(2):361-372.

Walker RE. Marine light field statistics. New York, USA: Wiley-Interscience; 1994.

Yudovsky D, Pilon L. Simple and accurate expressions for diffuse reflectance of semiinfinite and two-layer absorbing and scattering media. Appl Opt 2009;48(35):66706683.

Zege EP, Ivanov AP, Katsev IL. Image transfer through a medium. New York, USA: Springer-Verlag; 1991. 